\title{
The applicability of far-infrared fine-structure lines as star formation rate tracers over wide ranges of metallicities and galaxy types ${ }^{\star}$
}

Ilse De Looze ${ }^{1}$, Diane Cormier ${ }^{2}$, Vianney Lebouteiller ${ }^{3}$, Suzanne Madden ${ }^{3}$, Maarten Baes ${ }^{1}$, George J. Bendo ${ }^{4}$, Médéric Boquien $^{5}$, Alessandro Boselli ${ }^{6}$, David L. Clements ${ }^{7}$, Luca Cortese ${ }^{8,9}$, Asantha Cooray ${ }^{10,11}$, Maud Galametz ${ }^{8}$, Frédéric Galliano ${ }^{3}$, Javier Graciá-Carpio ${ }^{12}$, Kate Isaak ${ }^{13}$, Oskar Ł. Karczewski ${ }^{14}$, Tara J. Parkin ${ }^{15}$, Eric W. Pellegrini ${ }^{16}$, Aurélie Rémy-Ruyer ${ }^{3}$, Luigi Spinoglio ${ }^{17}$, Matthew W. L. Smith ${ }^{18}$, and Eckhard Sturm ${ }^{12}$

1 Sterrenkundig Observatorium, Universiteit Gent, Krijgslaan 281 S9, 9000 Gent, Belgium e-mail: Ilse.DeLooze@ugent.be

2 Zentrum für Astronomie der Universität Heidelberg, Institut für Theoretische Astrophysik, Albert-Ueberle Str. 2, 69120 Heidelberg, Germany

${ }^{3}$ Laboratoire AIM, CEA, Université Paris VII, IRFU/Service d'Astrophysique, Bat. 709, 91191 Gif-sur-Yvette, France

${ }^{4}$ UK ALMA Regional Centre Node, Jodrell Bank Centre for Astrophysics, School of Physics and Astronomy, University of Manchester, Oxford Road, Manchester M13 9PL, UK

5 Institute of Astronomy, University of Cambridge, Madingley Road, Cambridge CB3 OHA, UK

${ }^{6}$ Laboratoire d'Astrophysique de Marseille - LAM, Université Aix-Marseille \& CNRS, UMR7326, 38 rue F. Joliot-Curie, 13388 Marseille CEDEX 13, France

7 Astrophysics Group, Imperial College, Blackett Laboratory, Prince Consort Road, London SW7 2AZ, UK

8 European Southern Observatory, Karl Schwarzschild Str. 2, 85748 Garching, Germany

9 Centre for Astrophysics and Supercomputing, Swinburne University of Technology, PO Box 218, Hawthorn VIC 3122, Australia

10 Department of Physics \& Astronomy, University of California, Irvine CA 92697, USA

11 Division of Physics, Astronomy and Mathematics, California Institute of Technology, Pasadena CA 91125, USA

12 Max-Planck-Institute for Extraterrestrial Physics (MPE), Giessenbachstraße 1, 85748 Garching, Germany

13 ESA Research and Scientific Support Department, ESTEC/SRE-SA, Keplerlaan 1, 2201 AZ Noordwijk, The Netherlands

14 Department of Physics \& Astronomy, University of Sussex, Brighton BN1 9QH, UK

15 Department of Physics \& Astronomy, McMaster University, Hamilton, Ontario L8S 4M1, Canada

16 Department of Physics \& Astronomy, University of Toledo, Toledo OH 43606, USA

17 Istituto di Astrofisica e Planetologia Spaziali, INAF-IAPS, Via Fosso del Cavaliere 100, 00133 Roma, Italy

18 School of Physics and Astronomy, Cardiff University, Queens Buildings, The Parade, Cardiff CF24 3AA, UK

Received 14 August 2013 / Accepted 7 May 2014

\section{ABSTRACT}

Aims. We analyze the applicability of far-infrared fine-structure lines [CII] $158 \mu \mathrm{m}$, [OI] $63 \mu \mathrm{m}$, and [OIII] $88 \mu \mathrm{m}$ to reliably trace the star formation rate (SFR) in a sample of low-metallicity dwarf galaxies from the Herschel Dwarf Galaxy Survey and, furthermore, extend the analysis to a broad sample of galaxies of various types and metallicities in the literature.

Methods. We study the trends and scatter in the relation between the SFR (as traced by GALEX FUV and MIPS $24 \mu \mathrm{m}$ ) and farinfrared line emission, on spatially resolved and global galaxy scales, in dwarf galaxies. We assemble far-infrared line measurements from the literature and infer whether the far-infrared lines can probe the SFR (as traced by the total infrared luminosity) in a variety of galaxy populations.

Results. In metal-poor dwarfs, the $[\mathrm{OI}]_{63}$ and $[\mathrm{OIII}]_{88}$ lines show the strongest correlation with the SFR with an uncertainty on the SFR estimates better than a factor of 2, while the link between [CII] emission and the SFR is more dispersed (uncertainty factor of 2.6). The increased scatter in the $S F R-L_{[\mathrm{CII}]}$ relation toward low metal abundances, warm dust temperatures, and large filling factors of diffuse, highly ionized gas suggests that other cooling lines start to dominate depending on the density and ionization state of the gas. For the literature sample, we evaluate the correlations for a number of different galaxy populations. The $[\mathrm{CII}]$ and $[\mathrm{OI}]_{63} \mathrm{lines}$ are considered to be reliable SFR tracers in starburst galaxies, recovering the star formation activity within an uncertainty of factor 2 . For sources with composite and active galactic nucleus (AGN) classifications, all three FIR lines can recover the SFR with an uncertainty factor of 2.3. The SFR calibrations for ultra-luminous infrared galaxies (ULIRGs) are similar to starbursts/AGNs in terms of scatter but offset from the starburst/AGN SFR relations because of line deficits relative to their total infrared luminosity. While the number of detections of the FIR fine-structure lines is still very limited at high redshift for $[\mathrm{OI}]_{63}$ and $[\mathrm{OIII}]_{88}$, we provide an SFR calibration for [CII].

Key words. Galaxy: abundances - galaxies: dwarf - galaxies: ISM - galaxies: star formation

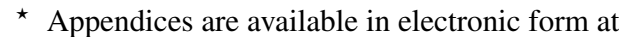

http://www. aanda.org 


\section{Introduction}

Star formation encompasses the birth of new stars through the fragmentation and contraction of cold, dense molecular gas, hereby recycling the interstellar medium (ISM) content of galaxies (McKee \& Ostriker 2007). Knowing the instantaneous level of star formation (i.e., the star formation rate) not only sheds light on the conditions in the ISM, but also on the evolution of galaxies and their formation processes. If we want to understand the physical processes that control galaxy evolution, being able to probe the star formation activity is of great importance. Tracing back star formation in some of the first objects in the early Universe until the present day could even allow us to probe the star formation activity throughout cosmic times.

Since the main coolants in metal-rich galaxies such as the Milky Way are mostly metal-based ([CII], [OI], [OIII], CO, dust), the metal abundance is considered a fundamental parameter in the regulation of star formation through its influence on the initial cooling of diffuse gas (e.g., Glover \& Clark 2014) and the survival of clouds through shielding. Star formation in the early Universe is considered to differ significantly from the present day's gas consumption in galaxies. Because of the extremely low metal abundances in the early Universe, the gas coolants and initiation processes of star formation were likely to be different from star formation conditions in the local Universe (e.g., Ly $\alpha$ cooling becomes more important). Nearby low-metallicity galaxies might be important laboratories to investigate the connection between the chemical enrichment and star formation processes. Although the present day metal-poor dwarfs will have experienced some evolution throughout cosmic time (e.g., Hodge et al. 1991; Tosi et al. 2007), their slow chemical evolution makes them important testbeds to understand metallicity effects potentially applicable to galaxies in the early Universe.

Star formation rates (SFRs) on global galaxy scales are typically estimated from scaling relations between diagnostic tracers of the star formation activity, and calibrated against the most up to date stellar population synthesis models and characterization of the initial mass function (IMF). Up to the present day, the most widely used SFR diagnostics have been based on continuum bands and optical/near-IR emission lines (see Kennicutt 1998; and Kennicutt \& Evans 2012, for a detailed overview). The brightest cooling lines in the atomic and molecular medium are emitted from mid-infrared to radio wavelengths, which have been probed extensively with the Herschel Space Observatory (Pilbratt et al. 2010). Follow-up is guaranteed with SOFIA in the local Universe or, at high spatial resolution, with groundbased interferometers such as the Atacama large millimeter array (ALMA) and the future northern extended millimeter array (NOEMA) in the high-redshift Universe. These facilities open up a whole new spectral window, which favors the use of far-infrared (FIR) and submillimeter (submm) continuum bands (e.g., PACS $70 \mu \mathrm{m}$, Li et al. 2013) and emission lines as SFR diagnostics across a large variety of galaxy populations.

Here, we investigate the utility of the three brightest finestructure cooling lines, $[\mathrm{CII}],[\mathrm{OI}]_{63}$, and $[\mathrm{OIII}]_{88}$ (e.g., Hunter et al. 2001; Brauher et al. 2008; Cormier et al., in prep.), as tracers of the star formation activity in a sample of lowmetallicity dwarf galaxies from the Herschel Dwarf Galaxy Survey (Madden et al. 2013). We, furthermore, assess the influence of metallicity, which is an important parameter controlling star formation in galaxies, constraining the reservoir of dust grains for the formation of molecules and regulating the attenuation of the FUV photons necessary for shielding molecules.
The [CII] $157.74 \mu \mathrm{m}$ line has been put forward as a potential powerful tracer of the star formation activity in the nearby as well as the more distant Universe (Stacey et al. 1991; Boselli et al. 2002; Stacey et al. 2010; De Looze et al. 2011; Sargsyan et al. 2012) and we, now, aim to expand this analysis to nearby, low-metallicity dwarf galaxies. This [CII] line is considered to be the dominant coolant for neutral atomic gas in the ISM (Tielens \& Hollenbach 1985a,b; Wolfire et al. 1995) and, therefore, among the brightest emission lines originating from star-forming galaxies (e.g., Stacey et al. 1991; Malhotra et al. 1997; Brauher et al. 2008). In particular, low-metallicity galaxies show exceptionally strong [CII] line emission (e.g., Poglitsch et al. 1995; Madden et al. 1997; Madden 2000; Hunter et al. 2001; Cormier et al. 2010; Israel \& Maloney 2011). Carbon has an ionization potential of $11.3 \mathrm{eV}$ (compared to $13.6 \mathrm{eV}$ for hydrogen), implying that line emission can originate from neutral and ionized gas components (see Table 1). A changing contribution of different gas phases on global scales can prevent a correlation between the [CII] line emission and the level of star formation. The excitation of $\mathrm{C}^{+}$atoms might, furthermore, saturate at high temperatures, where the line becomes insensitive to the intensity of the radiation field at temperatures well above the excitation potential (Kaufman et al. 1999). The [CII] emission can also saturate in neutral gas media with hydrogen densities $n_{\mathrm{H}} \gtrsim 10^{3} \mathrm{~cm}^{-3}$, where the recombination of $\mathrm{C}^{+}$into neutral carbon and, eventually, CO molecules is favored (Kaufman et al. 1999). Selfabsorption can also affect the [CII] line excitation in high column densities of gas $\left(N_{\mathrm{H}} \sim 4 \times 10^{22} \mathrm{~cm}^{-2}\right.$, Malhotra et al. 1997). Although the [CII] line is usually not affected by extinction, optical depth effects might become important in extreme starbursts (Luhman et al. 1998; Helou 2000) and edge-on galaxies (Heiles 1994). On top of this, deficits in the [CII]/FIR ratio toward warm dust temperatures (Crawford et al. 1985; Stacey et al. 1991; Malhotra et al. 1997, 2001; Luhman et al. 2003; Verma et al. 2005; Brauher et al. 2008; Graciá-Carpio et al. 2011; Croxall et al. 2012; Díaz-Santos et al. 2013; Farrah et al. 2013) suggest that the [CII] conditions might be different in galaxies that are offset from the main sequence of star-forming galaxies. Since carbon can also be significantly depleted on carbon-rich dust grains, the use of $\mathrm{O}$-based gas tracers might be preferred instead.

The $[\mathrm{OI}]_{63}$ line has a critical density $n_{\text {crit, } \mathrm{H}} \sim 5 \times 10^{5} \mathrm{~cm}^{-3}$ and upper state energy $E_{\mathrm{u}} / k \sim 228 \mathrm{~K}$ (see Table 1), which makes it an efficient coolant in dense and/or warm photodissociation regions (PDRs). Although generally observed to be the second brightest line (after $[\mathrm{CII}]$ ), the $[\mathrm{OI}]_{63}$ line is observed to be brighter in galaxies with warm FIR colors and/or high gas densities (Malhotra et al. 2001; Brauher et al. 2008; Lebouteiller et al. 2012). The applicability of [OI $]_{63}$ as an SFR calibrator might, however, be hampered by self-absorption (Kraemer et al. 1996; Poglitsch et al. 1996), optical depth effects (more so than [CII]) and the possible excitation of [OI $]_{63}$ through shocks (Hollenbach $\&$ McKee 1989). In the situation that the gas heating is not longer dominated by the photoelectric effect but has an important contribution from other heating mechanisms (e.g., mechanical heating, soft X-ray heating), the origin of the line emission might differ from the paradigm of warm and/or dense PDRs. A possible origin of $[\mathrm{OI}]_{63}$ emission that is different from PDRs can be, in particular, expected in the most metal-poor galaxies characterized by overall low metal content and diminished polycyclic aromatic hydrocarbon (PAH) abundances.

With an energy of the upper state $E_{\mathrm{u}} / k \sim 163 \mathrm{~K}$, critical density $n_{\text {crit, }} \sim 510 \mathrm{~cm}^{-3}$ and a high ionization potential of $35.1 \mathrm{eV}$ for $\mathrm{O}^{+}$, the $[\mathrm{OIII}]_{88}$ line originates from diffuse, highly ionized 
Ilse De Looze et al.: The applicability of far-infrared fine-structure lines as star formation rate tracers

Table 1. Excitation conditions of the fine-structure lines $[\mathrm{CII}],[\mathrm{OI}]_{63}$, and $[\mathrm{OIII}]_{88}$, with Col. 2 providing the ionization potential (IP) to create the species.

\begin{tabular}{lcccccc}
\hline \hline Line & $\begin{array}{c}I P^{a} \\
{[\mathrm{eV}]}\end{array}$ & $\begin{array}{c}E_{\mathrm{u}} / k \\
{[\mathrm{~K}]}\end{array}$ & $\begin{array}{c}n_{\text {crit, }} \\
{\left[\mathrm{cm}^{-3}\right]}\end{array}$ & $\begin{array}{c}n_{\text {crit, }} \\
{\left[\mathrm{cm}^{-3}\right]}\end{array}$ & Ref & Origin? \\
\hline$[\mathrm{CII}]$ & 11.3 & 91 & $1.6 \times 10^{3}$ & 44 & 1 & PDRs, diffuse HI clouds, diffuse ionized gas, HII regions \\
{$[\mathrm{OI}]_{63}$} & - & 228 & $5 \times 10^{5}$ & - & 2 & warm and/or dense PDRs \\
{$[\mathrm{OIII}]_{88}$} & 35.1 & 163 & - & 510 & 3 & low excitation, highly ionized gas \\
\hline
\end{tabular}

Notes. Columns 3-5 give the energy of the upper state and critical density for line excitation through collisions with hydrogen atoms and electrons, calculated for a kinetic temperature $T_{\text {kin }}=8000 \mathrm{~K}$. Column 6 provides the reference to the literature work reporting the excitation conditions of each fine-structure line. Column 7 specifies the ISM phase(s) from which the lines might originate. ${ }^{(a)}$ The ionization potential refers to the ionization energy of an atom to create the species in Col. 1. For example, the ionization potential of $35.1 \mathrm{eV}$ for [OIII $]_{88}$ indicates the energy required to remove another electron from $\mathrm{O}^{+}$and, thus, ionize $\mathrm{O}^{+}$in order to create $\mathrm{O}^{++}$.

References. (1) Goldsmith et al. (2012); (2) Tielens \& Hollenbach (1985a); (3) Aggarwal \& Keenan (1999).

regions near young $\mathrm{O}$ stars. Ionized gas tracers such as $[\mathrm{OIII}]_{88}$ might gain in importance in low-metallicity environments where PDRs occupy only a limited volume of the ISM judging from their weak PAH emission (Boselli et al. 2004; Engelbracht et al. 2005; Jackson et el. 2006; Madden et al. 2006; Draine et al. 2007; Engelbracht et al. 2008; Galliano et al. 2008) and low CO abundance (Poglitsch et al. 1995; Israel et al. 1996; Madden et al. 1997; Israel \& Maloney 2011). Based on our Herschel observations, we focus on [OIII $]_{88}$ as ionized gas tracer. Because of the low critical densities for $[\mathrm{OIII}]_{88}$ excitation with electrons, other lines (e.g., [OIII $]_{52}$ but also optical lines such as [OIII] $5007 \AA$ and $\mathrm{H} \alpha$ ) will likely dominate the cooling of ionized gas media for intermediate and high gas densities. The brightness of the $[\mathrm{OIII}]_{88}$ emission line in metal-poor galaxies (Cormier et al., in prep.), however, hints at an SFR tracer with great potential for the high-redshift Universe.

On top of the SFR calibrations for single FIR lines, we try to combine the emission of $[\mathrm{CII}],[\mathrm{OI}]_{63}$, and $[\mathrm{OIII}]_{88}$ lines to trace the SFR. The total gas cooling in galaxies scales with the SFR assuming that the ISM is in thermal equilibrium and the total cooling budget balances the gas heating. Therefore, any FIR fine-structure line can be considered a reliable tracer of the SFR if it plays an important role in the cooling of gas that was heated by young stellar photons. Other heating mechanisms unrelated to the UV radiation (e.g., mechanical heating, cosmic ray heating, and X-ray heating) and, thus, not directly linked to SFR, might disperse this link. Ideally, we combine the emission of several cooling lines in the ultraviolet/optical (e.g., Ly $\alpha, \mathrm{H} \alpha$, [OIII] $5007 \AA$ A) and infrared (e.g., [NeIII] $16 \mu \mathrm{m}$, [SIII] 19, $33 \mu \mathrm{m}$, [CII] $158 \mu \mathrm{m},[\mathrm{OI}] 63,145 \mu \mathrm{m},[\mathrm{NII}] 122,205 \mu \mathrm{m}$, [OIII] 52, $88 \mu \mathrm{m},[\mathrm{NIII}] 57 \mu \mathrm{m}$, and [SiII] $35 \mu \mathrm{m}$ ) wavelength domains to cover the total gas cooling budget in galaxies. Given our focus on the FIR coolants, we attempt to obtain a more complete picture of the overall cooling budget by combining the $[\mathrm{CII}],[\mathrm{OI}]_{63}$, and $[\mathrm{OIII}]_{88}$ lines.

The Herschel Dwarf Galaxy Survey (DGS) and observations are presented in Sect. 2, together with the acquisition and processing of ancillary data, which will be used as reference SFR calibrators. In Sect. 3, we take advantage of the high spatial resolution attained for the most nearby galaxies to study the trends and scatter in the spatially resolved relation between the SFR and FIR line emission. The SFR calibrations and trends with the scatter in the $S F R-L_{\text {line }}$ relations based on global galaxy measurements for the entire DGS sample are presented in Sect. 4. SFR calibrations for each of the fine-structure lines [CII], [OI $]_{63}$ and $[\mathrm{OIII}]_{88}$ are derived for a selection of different galaxy populations in Sect. 5. In Sect. 6, we draw our conclusions. Appendix A discusses the applicability of different unobscured (Sect. A.2) and obscured (Sect. A.3) indicators as reference SFR tracers for the low-metallicity DGS sample. Appendix A.4 presents a comparison between Herschel and ISO spectroscopy. Tables with source information, measurements of reference SFR calibrators and FIR lines are presented in Appendix B for the DGS sample, the literature sample of galaxies with starburst, composite, or AGN classifications and high-redshift sources.

\section{Dwarf Galaxy Survey}

\subsection{Sample characteristics}

The Dwarf Galaxy Survey (DGS, Madden et al. 2013) is a Herschel guaranteed time key program, gathering the photometry from the Photodetector Array Camera and Spectrometer (PACS, Poglitsch et al. 2010) and the Spectral and Photometric Imaging Receiver (SPIRE, Griffin et al. 2010), and PACS spectroscopy of 50 dwarf galaxies in $230 \mathrm{~h}$. The sample was selected to cover a wide range in metallicities from $12+\log (\mathrm{O} / \mathrm{H})=8.43$ $\left(\right.$ He $\left.2-10,0.55 Z_{\odot}\right)$ down to $7.14\left(\mathrm{IZw} 18,0.03 Z_{\odot}\right)^{1}$. The sample selection was furthermore optimized to maximize the availability of ancillary data. With distances ranging from several kpc to $191 \mathrm{Mpc}$, the Dwarf Galaxy Survey observes the line emission of more distant galaxies within a single beam (PACS beams have full width at half maximum (FWHM) of $11.5^{\prime \prime}, 9.5^{\prime \prime}$ and $9.5^{\prime \prime}$ for $[\mathrm{CII}],[\mathrm{OI}]_{63}$ and $[\mathrm{OIII}]_{88}$ ), while the extended FIR line emission from the brightest star-forming regions is mapped in the most nearby galaxies by the Herschel Space Observatory. More details about the sample selection as well as a description of the scientific goals of the survey are outlined in Madden et al. (2013).

\subsection{Herschel data}

Spectroscopic mapping of the [CII] $158 \mu \mathrm{m}$ line was performed for 48 galaxies $^{2}$, among which most galaxies were also covered in [OIII] $88 \mu \mathrm{m}$ (43 out of 48 galaxies) and [OI] $63 \mu \mathrm{m}$ (38 out of 48 galaxies). Far-infrared fine-structure lines [OI] $145 \mu \mathrm{m}$, [NII] $122 \mu \mathrm{m},[\mathrm{NII}] 205 \mu \mathrm{m}$, and [NIII] $57 \mu \mathrm{m}$ were probed in a subsample of the brightest galaxies (see the histogram in Fig. 4 of Madden et al. 2013). An overview of the spectroscopy

\footnotetext{
1 Oxygen abundances, which are used here to constrain metallicities, are calculated from optical line intensities following the prescriptions in Pilyugin \& Thuan (2005), assuming a solar oxygen abundance $\mathrm{O} / \mathrm{H}_{\odot}=$ $4.9 \times 10^{-4}$, or $12+\log (\mathrm{O} / \mathrm{H})_{\odot}=8.7$ (Asplund et al. 2009).

2 The DGS galaxies UGCA 20 and Tol 0618-402 were not observed with the PACS spectrometers on board Herschel.
} 


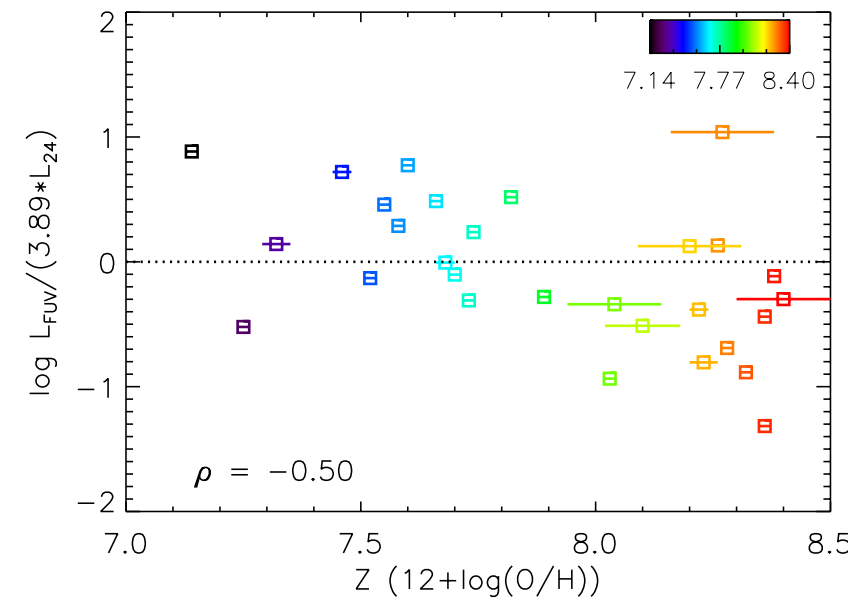

Fig. 1. Ratio of the unobscured $\left(L_{\mathrm{FUV}}\right)$ versus obscured star formation $\left(3.89^{*} L_{24}\right)$, as a function of oxygen abundance, $12+\log (\mathrm{O} / \mathrm{H})$, for the DGS sample. The multiplicative factor of 3.89 in the denominator arises from the calibration coefficient that corrects the observed FUV emission for extinction following Hao et al. (2011) (i.e., $L_{\mathrm{FUV}}$ (corr) $=$ $\left.L_{\mathrm{FUV}}(\mathrm{obs})+3.89 \times L_{24}\right)$. Galaxies are color-coded according to metallicity with increasing oxygen abundances ranging from black then blue, green and yellow to red colors. Data points correspond to global galaxy fluxes.

observations, data reduction, line flux measurements, and line ratios is provided in Cormier et al. (in prep).

\subsection{Reference SFR diagnostic}

To establish the applicability of FIR lines to trace the SFR, we need to specify a reference star formation rate tracer. Typically, combinations of SF diagnostics are used to trace the unobscured and obscured fraction of star formation. Figure 1 shows the ratio of unobscured versus obscured star formation, as probed by GALEX FUV and MIPS $24 \mu \mathrm{m}$, respectively, i.e., our reference calibrators of the unobscured and obscured star formation (see later), as a function of oxygen abundance for the DGS sample. The Spearman's rank correlation coefficient, $\rho$, is computed from the IDL procedure r_correlate to quantify the degree of correlation between the oxygen abundance and the ratio of unobscuredversus-obscured star formation. Values of $\rho$ close to +1 and -1 are indicative of a strong correlation or anti-correlation, respectively, while values approaching 0 imply the absence of any correlation. For the low metallicity $(12+\log (\mathrm{O} / \mathrm{H}) \leq 7.8-7.9)$ galaxies of the DGS sample, the fraction of unobscured star formation starts to dominate (see the negative correlation in Fig. 1 with $\rho=-0.50$ ) and we, thus, need tracers of the unobscured and obscured fraction of star formation. The increased fraction of unobscured star formation toward lower metal abundances is in agreement with the drop in the ratio of the SFR estimated from the WISE band at $12 \mu \mathrm{m}$ versus the SFR from $\mathrm{H} \alpha$ emission, $S F R_{\mathrm{W} 3} / S F R_{\mathrm{H} \alpha}$, with decreasing metallicity reported in Lee et al. (2013), which was attributed to the low dust-to-gas ratios of metal-poor galaxies (e.g., Rémy-Ruyer et al. 2014), making the reprocessing of UV photons by dust inefficient (Schurer et al. 2009; Hwang et al. 2012).

In this paper, we choose GALEX FUV and MIPS $24 \mu \mathrm{m}$ as reference SFR tracers to probe the unobscured and obscured star formation component, respectively, and we rely on the SFR calibrations presented by Hao et al. (2011) and Murphy et al. (2011 see Table A.1). In Sect. A of the appendix, we motivate this choice of reference SFR calibrators by comparing different unobscured (GALEX FUV, H $\alpha$ ) and obscured (IRAC8 $\mu \mathrm{m}$, MIPS $\left.24 \mu \mathrm{m}, L_{\mathrm{TIR}}, 1.4 \mathrm{GHz}\right)$ SFR tracers for the DGS galaxy sample. Among the unobscured SFR diagnostics, we argue that the $\mathrm{H} \alpha$ line can provide better SFR estimates compared to FUV due to the limited range of ages to which the SFR calibration for $\mathrm{H} \alpha(\sim 10 \mathrm{Myr})$ is sensitive compared to FUV (100 Myr). Given the bursty star formation histories and small sizes of dwarf galaxies, the underlying assumption of continuous star formation activity during the age range of the SFR calibrator is not fulfilled, which becomes worse for the FUV emission probing a longer timescale of star formation activity. The unavailability of $\mathrm{H} \alpha$ maps prevents us from determining the $\mathrm{H} \alpha$ emission that corresponds to the galaxy regions covered in our Herschel observations. The FUV emission is instead used as a reference SFR calibrator, but note that FUV might underestimate the SFR by $50 \%$ as compared to $\mathrm{H} \alpha$ (see Sect. A.2). The analysis in Sect. A.3 shows that IRAC $8 \mu \mathrm{m}, L_{\mathrm{TIR}}$, and $L_{1.4 \mathrm{GHz}}$ are unreliable SFR tracers of the obscured SF fraction due to the dependence of PAH abundance on metallicity, the peculiar SED shapes and/or the burstiness of the star formation histories of metal-poor dwarfs. Although the MIPS $24 \mu \mathrm{m}$ band emission might not be entirely free of metallicity effects and/or variations in (very small) grain abundance, the analysis of Sect. A.3 shows that MIPS $24 \mu \mathrm{m}$ is linked more closely to the SFR over wide ranges of metallicity compared to the other obscured SFR diagnostics.

GALEX FUV data are processed in a similar way to that outlined in Cortese et al. (2012). The data are background subtracted and corrected for Galactic extinction according to the recalibrated $A_{V}$ in Schlafly et al. (2011) from Schlegel et al. (1998), as reported on the NASA/IPAC Extragalactic Database (NED), and assuming an extinction law with $R_{V}=3.1$ from Fitzpatrick et al. (1999). Spitzer MIPS data have been retrieved from the Herschel Database in Marseille $\left(\mathrm{HeDaM}^{3}\right)$. Details of the data reduction of the ancillary MIPS data set can be found in Bendo et al. (2012b), along with aperture photometry techniques and results.

\section{Spatially resolved $S F R-L_{\text {line }}$ relation}

\subsection{Convolution and regridding of the maps}

The closest galaxies in the DGS sample were mapped with Herschel to cover most of the star-forming regions and, therefore, can be used to provide a spatially resolved interpretation of the SFR calibrations. For the spatially resolved analysis, we consider all sources at distances $D \leq 7.5 \mathrm{Mpc}$ with GALEX FUV and MIPS $24 \mu \mathrm{m}$ observations ${ }^{4}$. A visual inspection of the MIPS $24 \mu \mathrm{m}$ images shows that the mid-infrared emission from VII Zw 403, Mrk 209, UGC 4483, and NGC 625 is barely resolved with respect to the MIPS $24 \mu \mathrm{m}$ beam ( FWHM $\sim 6^{\prime \prime}$, Engelbracht et al. 2007), and, therefore, those galaxies are excluded from the sample for the spatially resolved analysis. We, therefore, end up with a subsample of seven well sampled galaxies with metal abundances varying from $0.10 Z_{\odot}$ (NGC 2366) to $0.38 Z_{\odot}(\mathrm{NGC} 1705)$.

All maps of the resolved subsample have been convolved from their native resolution $\left([\mathrm{OIII}]_{88}: 9.5^{\prime \prime},[\mathrm{OI}]_{63}: 9.5^{\prime \prime} ;\right.$ GALEX FUV: $6^{\prime \prime}$; MIPS $\left.24 \mu \mathrm{m}: 6^{\prime \prime}\right)$ to the $12^{\prime \prime}$ resolution of PACS at $160 \mu \mathrm{m}$ using the kernels presented in Aniano et al. (2011).

\footnotetext{
3 http://hedam.lam.fr/

4 The distance of 7.5 Mpc has been chosen as a fair compromise between the number of spatially resolved galaxies to be analyzed and the spatial resolution of about $100 \mathrm{pc}$, corresponding to the nominal pixel size of $3.1333^{\prime \prime}$ at $7.5 \mathrm{Mpc}$.
} 
Convolved images are rebinned to maps with pixel size corresponding to regions within galaxies of $114^{2} \mathrm{pc}^{2}$, i.e., the size of a nominal pixel of $3.1333^{\prime \prime}$ at $7.5 \mathrm{Mpc}$. We caution that pixels of this size are not independent due to the shape of the beam (FWHM of 12"), which is spread across several pixels within one galaxy. Since our main interest is the comparison of the behavior in the relations between the SFR and FIR line emission for different galaxies, we argue that a possible dependence of individual pixels for one specific galaxy will not severely affect the interpretation of our results. Given that the pixels defined this way correspond to a same physical scale within galaxies, the pixels provide a proxy for the surface density of the SFR, $\Sigma_{\mathrm{SFR}}$, and the FIR line surface density, $\Sigma_{\text {line }}$. Only pixels attaining surface brightness levels of signal-to-noise $(\mathrm{S} / \mathrm{N})$ higher than 5 are taken into consideration, neglecting the uncertainty from the calibration. Because of the proximity of NGC $6822(D=0.5 \mathrm{Mpc})$, the rebinning of pixels results in the absence of any region at sufficient $\mathrm{S} / \mathrm{N}$ level, limiting the spatially resolved galaxy sample to six objects. For NGC 1705, the $[\mathrm{OI}]_{63}$ line is not detected at sufficient $\mathrm{S} / \mathrm{N}$ level on spatially resolved scales.

\subsection{Observed trends}

Figure 2 shows the relation between the SFR and [CII], [OI $]_{63}$, and $[\mathrm{OIII}]_{88}$ line emission for the subsample of spatially resolved galaxies. Different galaxies are color-coded according to their oxygen abundance. Since abundances are determined for global galaxies, they may not give representative values for the metal abundance on spatially resolved scales. For example, the inefficient ISM mixing of nebular and neutral gas might cause deviations from this global metallicity value on kiloparsec scales (Roy \& Kunth 1995; Lebouteiller et al. 2004). Since the coverage in $[\mathrm{CII}]$ is larger with respect to the areas mapped in [OI $]_{63}$ and $[\mathrm{OIII}]_{88}$ and/or the $[\mathrm{CII}]$ line might attain higher $\mathrm{S} / \mathrm{N}$ levels in some areas, the number of pixels with $S / N>5$ is higher for [CII] (1274 pixels) than for [OI $]_{63}(602)$ and [OIII $]_{88}(605)$ lines.

The SFR calibrations of spatially resolved regions are determined from Levenberg-Marquardt least-squares fitting using the IDL procedure MPFITFUN, which is based on the nonlinear least-squares fitting package MPFIT (Markwardt 2009). The MPFITFUN procedure only accounts for the uncertainties on the SFR, which assumes that the line luminosities are errorfree. While the line measurements are obviously affected by uncertainties inherent to the calibration and/or line fitting techniques, we prefer to account for the uncertainties on the SFR in the fitting procedure since the precision of the SFR estimate is affected by uncertainties on the reference SFR calibrators as well as the inaccuracy inherent to the applied reference SFR calibrators and, therefore, often larger than the uncertainties on the line measurements. Table 2 summarizes the results of the fitted SFR calibrations:

$\log \Sigma_{\mathrm{SFR}}=\beta+\alpha * \log \Sigma_{\text {line }}$

where $\Sigma_{\text {line }}$ is the FIR line surface density in units of $L_{\odot} \mathrm{kpc}^{-2}$, $\Sigma_{\mathrm{SFR}}$ is the star formation rate in units of $M_{\odot} \mathrm{yr}^{-1} \mathrm{pc}^{-2}$, and $\alpha$ and $\beta$ represent the slope and intercept of the best fit. We require that parameter $\alpha$ can be determined at a $>5 \sigma$ significance level to determine that two quantities (i.e., SFR and FIR line surface density) are correlated.

The observed trends in Fig. 2, in combination with the $>5 \sigma$ significance of the fitted slope of each trend, suggest a correlation between the SFR and $[\mathrm{CII}],[\mathrm{OI}]_{63},[\mathrm{OIII}]_{88}$ line emission, which persists over almost two orders of magnitude in surface
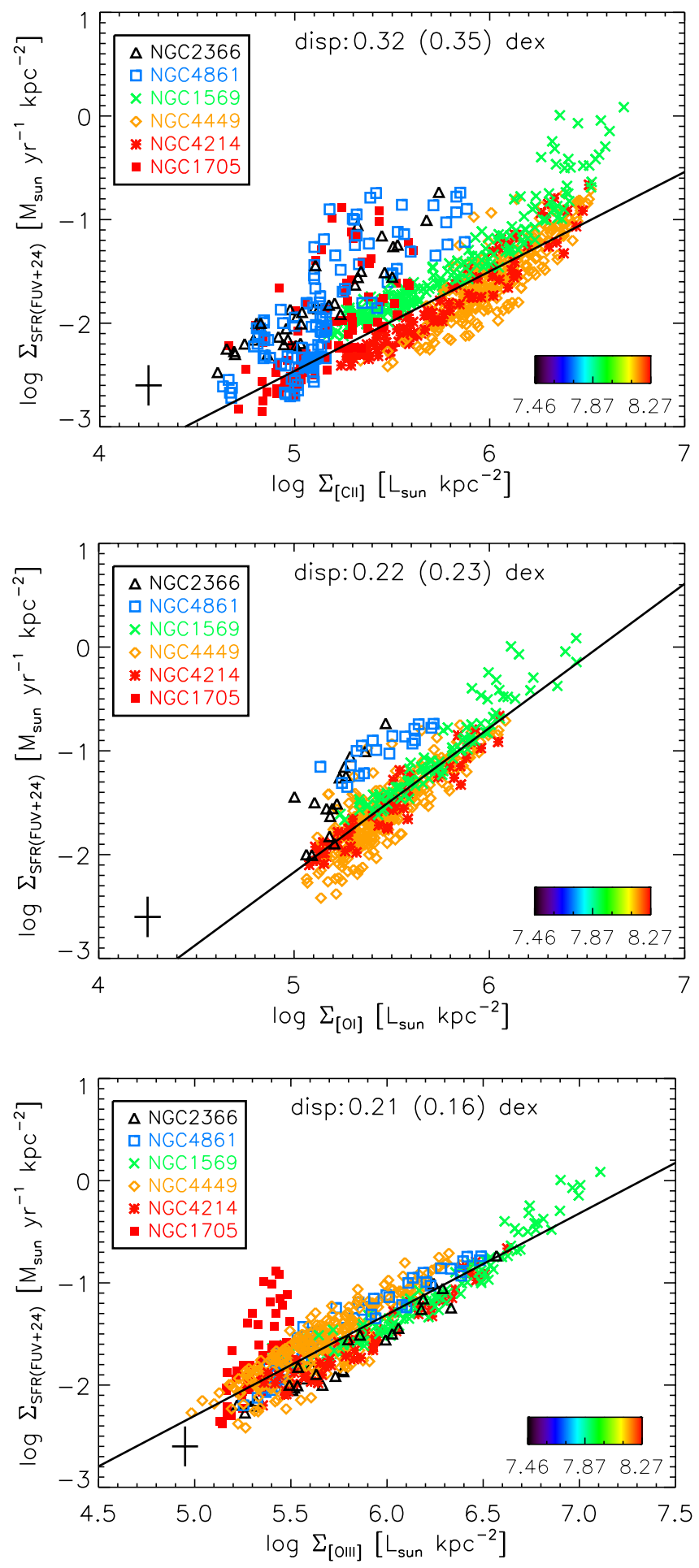

Fig. 2. Spatially resolved galaxy relation between surface densities of the SFR and [CII] (top), $[\mathrm{OI}]_{63}$ (middle), and [OIII $]_{88}$ (bottom) surface densities. The legend explains the symbols used for different galaxies with the color bar indicating the oxygen abundance. Representative error bars are indicated in the lower left corner. Uncertainties on the SFR include the errors on each of the SFR calibrators (GALEX FUV, MIPS $24 \mu \mathrm{m}$ ) as well as the average scatter in the calibrations used to convert to the SFR (see Table A.1). Uncertainties on the FIR line surface densities incorporate the errors due to line fitting as well as calibration uncertainties (taken conservatively as $\sim 30 \%$ ). The best fitting SFR calibration is presented as a solid, black line. The dispersion of data points around the SFR calibration is indicated at the top of each panel, with the number in parentheses indicating the scatter for the complete sample with $>5 \sigma$ detections for all three lines. 
density. The smallest dispersion $(0.21 \mathrm{dex})$ as well as the best constraint on the slope parameter with $S / N \sim 50$ is found for the $[\mathrm{OIII}]_{88}$ line, suggesting that the $[\mathrm{OIII}]_{88}$ line more tightly correlates with the SFR as compared to $[\mathrm{OI}]_{63}(0.22 \mathrm{dex})$ and [CII] (0.32 dex) lines on spatially resolved scales of $\sim 100 \mathrm{pc}$. Comparing different galaxies, we observe consistent trends with similar slopes between the FIR line emission and SFR. Toward brighter regions, the slope of the $S F R-L_{[\mathrm{CII}]}$ relation appears to get steeper in most galaxies, suggesting that the [CII] line is not the dominant coolant in dense star-forming regions, where other cooling lines $\left([\mathrm{OI}]_{63}\right.$ or $\left.[\mathrm{OIII}]_{88}\right)$ are favored given the density and ionization state of the gas (Lebouteiller et al. 2012). The steep slope $(\alpha=1.41)$ in the spatially resolved $S F R-L_{[\mathrm{OI}]}$ relation suggests a sudden drop in the $[\mathrm{OI}]_{63}$ emission for a decrease in star formation activity, while the flatter $\operatorname{SFR}-L_{[\mathrm{CII}]}(\alpha=0.93)$ and $S F R-L_{[\mathrm{OIII}]}(\alpha=1.01)$ relations indicate that the $[\mathrm{CII}]$ and $[\mathrm{OIII}]_{88}$ lines remain bright in regions of relatively low SFRs. Given the high upper state energy and critical density for $[\mathrm{OI}]_{63}$ (see Table 1), it is not surprising that bright $[\mathrm{OI}]_{63}$ emission only occurs in warm and/or dense star-forming regions, where we expect to find the highest level of star formation activity.

A few galaxies (NGC 1705, NGC 2366, and NGC 4861) show diverging behavior in some of the SFR relations. NGC 1705 demonstrates a peculiar behavior in the $S F R-L_{[\mathrm{CII}]}$ and $S F R-L_{[\mathrm{OIII}]}$ relations with weaker line emission relative to its star formation rate. NGC 1705 is a dwarf starburst galaxy dominated by a central super star cluster (SSC) straddled by two dusty off-nuclear regions offset by $\sim 250 \mathrm{pc}$ that dominate the $\mathrm{H} \alpha$, midand FIR emission of the galaxy (Cannon et al. 2006). The chemistry and heating of gas in the off-nuclear positions do not seem to be regulated directly by the central SSC (Cannon et al. 2006), but rather exposed to the emission of young, massive stars produced during a second starburst about 3 Myr ago, which was presumably induced by the expanding shell after the first central starburst (Annibali et al. 2009). The weak [OIII $]_{88}$ emission originates from the eastern dust complex, which shows bright [CII] and $\mathrm{PAH}$ emission, suggesting that the deviation for the eastern regions of NGC 1705 could be due to a radiation field not strong enough to excite $[\mathrm{OIII}]_{88}$ (requiring massive $\mathrm{O6}$ and earlier-type stars). The western dust region does show bright $[\mathrm{OIII}]_{88}$ emission, but has weak [CII] and PAH emission. We, therefore, argue that the western dust complex is exposed to a harder radiation field, capable of destroying PAHs and ionizing the majority of the gas, which makes [OIII] $]_{88}$ a more efficient coolant. The nondetection of $[\mathrm{OI}]_{63}$, which is an efficient coolant of dense PDRs (see Table 1), might suggest rather diffuse ISM regions in the western and eastern dust emission complexes. Alternatively, optical depth effects might play a role in the two dusty off-nuclear regions. The behavior of NGC 2366 and NGC 4861 is mainly divergent in the $S F R-L_{[\mathrm{CII}]}$ and $S F R-L_{[\mathrm{OI}]}$ relations, whereas the $[\mathrm{OIII}]_{88}$ emission correlates remarkably well with the SFR, suggesting that the gas around massive star clusters is mostly ionized and that PDRs only occupy a limited volume of the ISM.

The different galaxies cover a wider range in the $S F R-\Sigma_{\text {line }}$ relations than is observed within one single object, suggesting that the dispersion in the $S F R-\Sigma_{\text {line }}$ relations is driven by the diversity on global galaxy scales rather than variations in the ISM conditions within individual objects. The $S F R-L_{[\mathrm{CII}]}$ relation is most affected by this different behavior of galaxies with a dispersion of $0.32 \mathrm{dex}$ around the fitted SFR calibration. More metal-rich galaxies show lower star formation rates as traced by FUV+MIPS $24 \mu \mathrm{m}$ than those predicted by our SFR calibration given their [CII] emission. Sources with lower metal abundances, on the other hand, preferentially populate the part of the

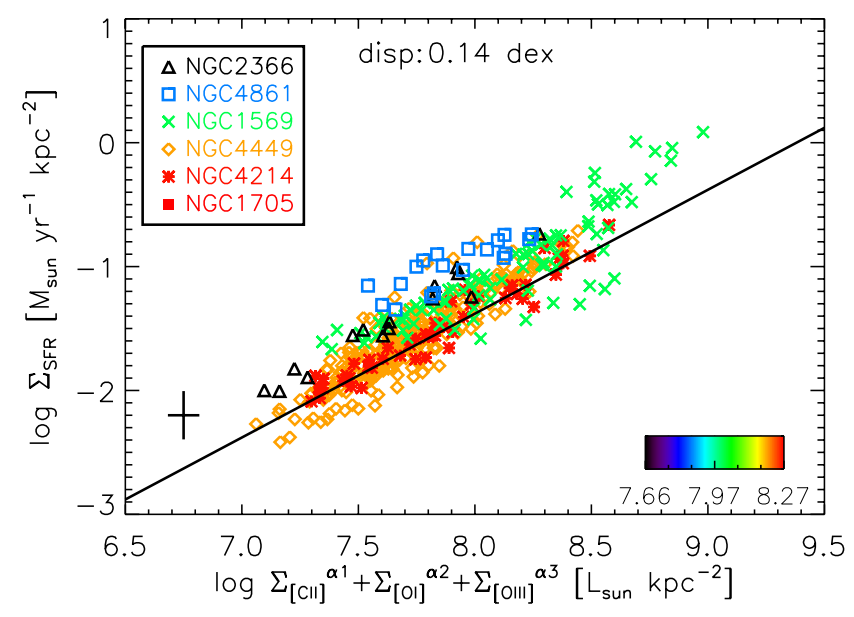

Fig. 3. Spatially resolved galaxy relation between surface densities of the SFR and a combination of $[\mathrm{CII}],[\mathrm{OI}]_{63}$, and $[\mathrm{OIII}]_{88}$ surface densities. The image format is the same as explained in Fig. 2.

plot representative of weaker [CII] emission and/or higher levels of star formation. Since the SFR based on FUV+MIPS $24 \mu \mathrm{m}$ might be underestimated in more metal-poor dwarfs relative to the SFR calibrators H $\alpha+$ MIPS $24 \mu \mathrm{m}$ (see Sect. A.2), the offset of metal-poor dwarf galaxies in the $S F R-\Sigma_{\text {line }}$ relations might even be more pronounced. With a dispersion of 0.22 and 0.21 dex in the $S F R-L_{[\mathrm{OI}]}$ and $S F R-L_{[\mathrm{OIII}]}$ relations, respectively, the fine-structure lines $[\mathrm{OI}]_{63}$ and $[\mathrm{OIII}]_{88}$ seem to depend less on the ISM conditions in galaxies and might, thus, be potentially better SFR indicators compared to [CII].

Given that we probe different ISM phases, even on spatially resolved scales of $\sim 100 \mathrm{pc}$, we try to better approximate the overall cooling budget through FIR lines by combining the surface densities of $[\mathrm{CII}],[\mathrm{OI}]_{63}$ and $[\mathrm{OIII}]_{88}$. For the combination of several FIR lines, we attempt to fit an SFR function of the form:

$\log \Sigma_{\mathrm{SFR}}=\beta+\log \left(\Sigma_{[\mathrm{CII}]}^{\alpha_{1}}+\Sigma_{[\mathrm{OI}]}^{\alpha_{2}}+\Sigma_{[\mathrm{OIII}]}^{\alpha_{3}}\right)$

where $\Sigma_{\text {line }}$ is the FIR line surface density in units of $L_{\odot} \mathrm{kpc}^{-2}$, $\Sigma_{\mathrm{SFR}}$ is the star formation rate in units of $M_{\odot} \mathrm{yr}^{-1} \mathrm{pc}^{-2}$ and $\left(\alpha_{1}\right.$, $\alpha_{2}, \alpha_{3}$ ), and $\beta$ represent the slopes and intercept of the best fit.

In the fitting procedure, the different data points are equally weighted and the parameters $\left(\alpha_{1}, \alpha_{2}, \alpha_{3}\right)$ of the slopes are constrained to positive numbers. Similar functions are defined to fit combinations of two FIR lines. Best fitting parameters (including calibration coefficients and dispersion) are only presented in Table 2 for line combinations that improved on the dispersion in the SFR calibrations for single FIR lines. By combining a number of FIR lines, we are able to significantly reduce the scatter (see Fig. 3), confirming the hypothesis that the total gas cooling balances the gas heating under the condition of local thermal equilibrium. This, furthermore, suggests that the specific processes that regulate the cooling in the different gas phases are of minor importance. Although the primary heating mechanisms are very different in the neutral gas phase (photoelectric effect and a variable contribution from cosmic rays and soft $\mathrm{X}$-ray heating), as compared to ionized gas media (photoionization processes), the main goal is to get access to the total heating due to young stellar emission, irrespective of the dominant heating mechanism in different gas phases, by probing the total cooling budget in galaxies. In particular, the combinations $[\mathrm{CII}]+[\mathrm{OIII}]_{88},[\mathrm{OI}]_{63}+[\mathrm{OIII}]_{88}$ and $[\mathrm{CII}]+[\mathrm{OI}]_{63}+[\mathrm{OIII}]_{88}$ provide accurate estimates of the SFR, 
Ilse De Looze et al.: The applicability of far-infrared fine-structure lines as star formation rate tracers

Table 2. Overview of the calibration coefficients for SFR calibrations based on the spatially resolved (top) and global galaxy (bottom) DGS sample.

\begin{tabular}{|c|c|c|c|c|}
\hline SFR calibrator & Number of data points & Slope & Intercept & $1 \sigma$ dispersion $[\mathrm{dex}]$ \\
\hline \multicolumn{5}{|c|}{ SFR calibration: spatially resolved DGS sample } \\
\hline [CII] & 1274 & $0.93 \pm 0.06$ & $-6.99 \pm 0.35$ & $0.32(0.35)$ \\
\hline$[\mathrm{OI}]_{63}$ & 602 & $1.41 \pm 0.04$ & $-9.19 \pm 0.23$ & $0.22(0.23)$ \\
\hline$[\mathrm{OIII}]_{88}$ & 605 & $1.01 \pm 0.02$ & $-7.33 \pm 0.12$ & $0.21(0.16)$ \\
\hline$[\mathrm{CII}]+[\mathrm{OIII}]_{88}$ & 605 & $(0.94 \pm 0.03,1.08 \pm 0.02)$ & $-7.82 \pm 0.14$ & $0.20(0.15)$ \\
\hline$[\mathrm{OI}]_{63}+[\mathrm{OIII}]_{88}$ & 441 & $(1.30 \pm 0.03,1.25 \pm 0.02)$ & $-9.01 \pm 0.15$ & 0.14 \\
\hline$[\mathrm{CII}]+[\mathrm{OI}]_{63}+[\mathrm{OIII}]_{88}$ & 441 & $(1.23 \pm 0.03,1.31 \pm 0.03,1.25 \pm 0.07)$ & $-9.38 \pm 0.16$ & 0.14 \\
\hline \multicolumn{5}{|c|}{ SFR calibration: global galaxy DGS sample } \\
\hline [CII] & 32 & $0.84 \pm 0.06$ & $-5.29 \pm 0.34$ & $0.38(0.40)$ \\
\hline$[\mathrm{OI}]_{63}$ & 26 & $0.94 \pm 0.05$ & $-6.37 \pm 0.29$ & 0.25 \\
\hline$[\mathrm{OIII}]_{88}$ & 28 & $0.92 \pm 0.05$ & $-6.71 \pm 0.33$ & $0.30(0.30)$ \\
\hline
\end{tabular}

Notes. The first column indicates the FIR fine-structure line(s) used in the SFR calibration, with the number of data points, slope, intercept and dispersion of the best-fit line presented in Cols. 2-5, respectively. In parentheses, we show the dispersion for the complete galaxy sample, i.e. galaxy regions or global galaxies which have $>5 \sigma$ detections for all three lines [CII], [OII $]_{63}$ and $[\mathrm{OIII}]_{88}$.

which suggests that the cooling in the neutral as well as ionized media needs to be probed to approximate the overall cooling budget in metal-poor galaxies and, thus, trace the star formation activity. However, heating mechanisms not directly linked to the recent star formation activity (e.g., soft X-ray heating, Silk \& Werner 1969, which might become substantial in extremely low-metallicity galaxies, such as IZw 18, Péquignot 2008 and Lebouteiller et al., in prep.) might disperse the link between the emission of cooling lines and the star formation rate (see also Sects. 3.3 and 4.2).

\subsection{Scatter in the $S F R-L_{\text {line }}$ relation}

In this section, we analyze what is driving the dispersion in the $S F R-L_{\text {line }}$ relations on spatially resolved scales within galaxies by analyzing the trends with several diagnostics for the physical conditions of the ISM. We quantify the dispersion in the SFR$L_{\text {line }}$ relations as the logarithmic distance between the SFR as estimated from the reference SFR calibration based on FUV and $24 \mu \mathrm{m}$ emission and the best-fit line for the spatially resolved $S F R-L_{\text {line }}$ relation.

First of all, we analyze a possible link between the scatter and FIR color as probed by the PACS $100 \mu \mathrm{m} / \mathrm{PACS} 160 \mu \mathrm{m}$ flux density ratio obtained from dust continuum observations, considered a proxy of the dust temperature (or grain charging) under the assumption that the emission in both bands is heated by the same radiation field. Although different radiation fields, originating from star-forming regions or diffuse interstellar radiation, have been shown to contribute to the emission in FIR and submillimeter wavebands (e.g., Bendo et al. 2012a), the dust emission in dwarf galaxies seems to be primarily due to heating by young stars, even at wavelengths $>160 \mu \mathrm{m}$ (Galametz et al. 2010; Bendo et al. 2012a).

Figure 4 displays the observed trends between FIR color and dispersion in the $S F R-L_{\text {line }}$ relations for [CII] (top panel) and [OI] (bottom panel). The data reduction of PACS 100 and $160 \mu \mathrm{m}$ photometry maps is described in Rémy-Ruyer et al. (2013). The dispersion in the $S F R-L_{[\mathrm{CII}]}$ relation $(\rho=0.67)$ clearly correlates with the FIR colors of galaxy regions in the sense that the fine-structure line [CII] does not seem well suited as an SFR indicator toward warm FIR colors. Lowmetallicity galaxies often have warm FIR colors (e.g., Thuan et al. 1999; Houck et al. 2004; Galametz et al. 2009, 2011; Rémy-Ruyer et al. 2013), which could explain why galaxies such as NGC 2366 and NGC 4861 show an offset in the $S F R-L_{[\mathrm{CII}]}$ relation compared to more metal-rich galaxies. For increasing dust temperatures, the grain charging parameter increases and, therefore, the photoelectric heating efficiency decreases, diminishing the line cooling. We observe a similar but more moderate correlation with FIR color for $[\mathrm{OI}]_{63}(\rho=0.51)$. Similar plots for $[\mathrm{OIII}]_{88}$ are not shown here, since the line emission arises from the ionized gas component, where photoionization processes dominate the heating and no physical motivation exists for a correlation with FIR color.

Figure 5 shows the behavior of the scatter in the $S F R-L_{[\mathrm{CII}]}$ relation as a function of FIR line ratios $[\mathrm{OI}]_{63} /[\mathrm{CII}]+[\mathrm{OI}]_{63}$ (top) and $[\mathrm{OIII}]_{88} /[\mathrm{CII}]+[\mathrm{OI}]_{63}$ (bottom). With an upper state energy $E_{\mathrm{u}} / k \sim 228 \mathrm{~K}$ and critical density $n_{\text {crit, } \mathrm{H}} \sim 5 \times 10^{5} \mathrm{~cm}^{-3}$ for [OI $]_{63}$ as compared to $T_{\text {exc }} \sim 91 \mathrm{~K}$ and $n_{\text {crit, } \mathrm{H}} \sim 1.6 \times 10^{3} \mathrm{~cm}^{-3}$ for $[\mathrm{CII}]$, the $[\mathrm{OI}]_{63} /[\mathrm{CII}]+[\mathrm{OI}]_{63}$ ratio can be interpreted as a proxy for the relative fraction of warm and/or dense gas, which increases toward higher values of $[\mathrm{OI}]_{63} /[\mathrm{CII}]+[\mathrm{OI}]_{63}$. Indeed, PDR models have shown that this ratio increases toward higher gas density and radiation field strength (Kaufman et al. 2006). With $[\mathrm{OIII}]_{88}$ emission originating from highly-ionized regions near young $\mathrm{O}$ stars, the $[\mathrm{OIII}]_{88} /[\mathrm{CII}]+[\mathrm{OI}]_{63}$ ratio can be interpreted as a proxy for the relative contribution of the ionized gas phase with higher values implying large filling factors of diffuse highly ionized gas with respect to neutral media. The latter interpretations of line ratios are based on the assumption that most of the [CII] emission arises from PDRs rather than diffuse ionized gas media and also that $[\mathrm{OI}]_{63}$ emission can be identified merely with PDRs. The interpretation of this line ratio might differ for low-metallicity galaxies, however, where the filling factors of PDRs are considered to be low based on the weak emission of several PDR tracers (e.g., PAH, CO). High $[\mathrm{OI}]_{63} /[\mathrm{CII}]+[\mathrm{OI}]_{63}$ line ratios in dust deficient objects might, thus, have a different origin than warm and/or dense PDRs. Péquignot (2008) have shown that low-ionization line emission in neutral gas media can be produced by a pseudo-PDR with similar lines as PDRs, but with soft X-rays as the dominant heating mechanism (see also Lebouteiller et al., in prep).

There is a clear correlation $(\rho=0.74)$ between the $[\mathrm{OI}]_{63} /[\mathrm{CII}]+[\mathrm{OI}]_{63}$ line ratios and the observed scatter in the $S F R-L_{[C I I]}$ relation indicating that regions with higher values of $[\mathrm{OI}]_{63} /[\mathrm{CII}]+[\mathrm{OI}]_{63}$ are offset in the SFR relation toward weaker [CII] emission for a certain SFR. This implies that [CII] is not the most appropriate SFR indicator in those regions. Higher values 

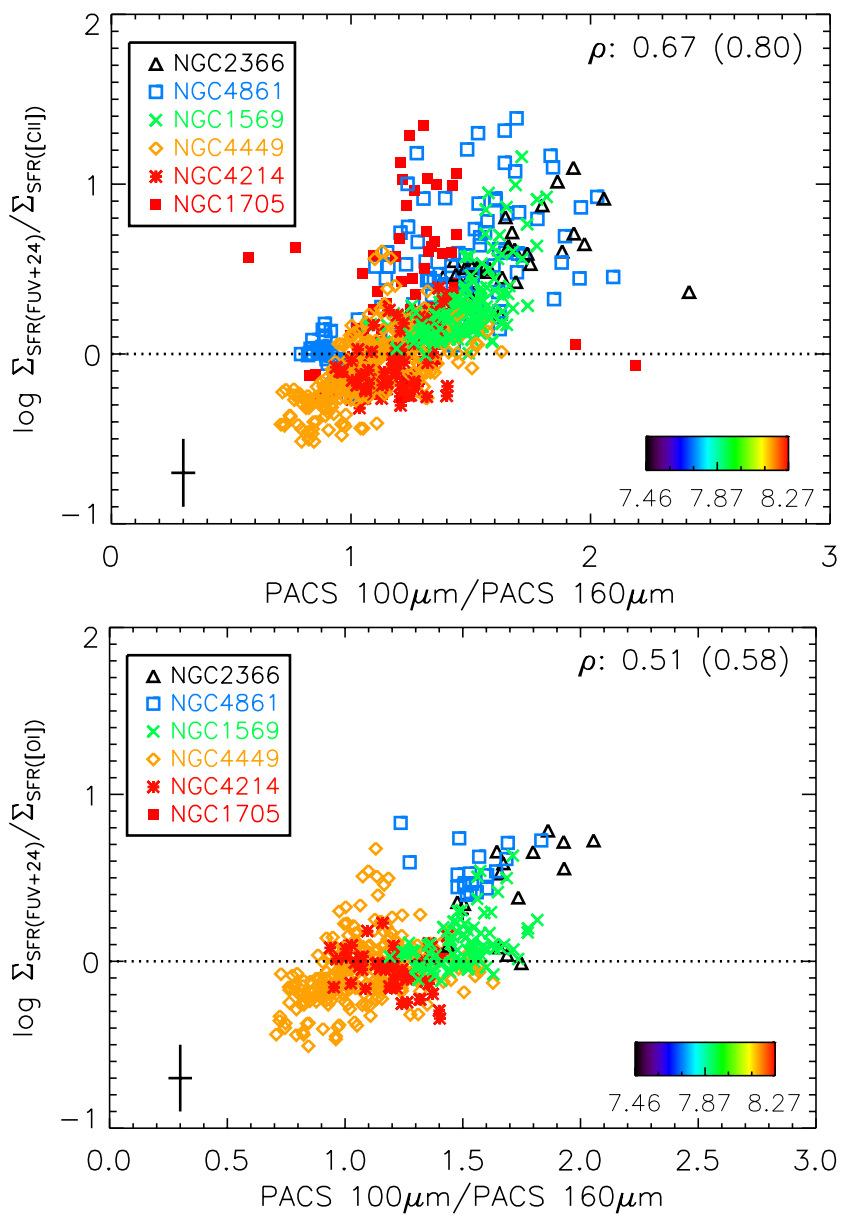

Fig. 4. Spatially resolved galaxy relation between the dispersion from the SFR calibrations for [CII] (top) and [OI $]_{63}$ (bottom) as a function of FIR color, i.e., PACS $100 \mu \mathrm{m} / \mathrm{PACS} 160 \mu \mathrm{m}$. The legend explains the symbols used for different galaxies with the color bar indicating the oxygen abundance. Representative error bars are indicated in the lower left corner. Uncertainties on the SFR include the errors on each of the SFR calibrators (GALEX FUV, MIPS $24 \mu \mathrm{m}$ ) as well as the average scatter in the reference calibration (see Table A.1). Uncertainties on the PACS line ratios incorporate the errors due to map-making as well as calibration uncertainties (5\%). The Spearman's rank correlation coefficients are presented in the top right corner. In parentheses, we show the dispersion for the complete galaxy sample, i.e., galaxy regions that have $>5 \sigma$ detections for all three lines $[\mathrm{CII}],[\mathrm{OI}]_{63}$, and $[\mathrm{OIII}]_{88}$.

of $[\mathrm{OI}]_{63} /[\mathrm{CII}]+[\mathrm{OI}]_{63}$ occur preferentially in galaxies of lower metal abundance (e.g., NGC 2366, NGC 4861), which might suggest that the importance of the photoelectric effect diminishes in dust deficient environments and other heatings mechanisms (e.g., soft X-ray heating) become more efficient.

With the $[\mathrm{OIII}]_{88} /[\mathrm{CII}]+[\mathrm{OI}]_{63}$ ratio (see bottom panel of Fig. 5) covering almost two orders of magnitude, we sample very distinct ISM conditions on hectoparsec scales within spatially resolved galaxies. The clear correlation of $[\mathrm{OIII}]_{88} /[\mathrm{CII}]+[\mathrm{OI}]_{63}$ with the scatter in the $S F R-L_{[\mathrm{CII}]}$ relation $(\rho=0.87)$ implies that the [CII] line is not a good tracer of the star formation activity in regions where the ionized gas phase occupies an important part of the ISM volume. Although the [CII] line could also regulate the cooling in ionized gas media in addition to being the dominant coolant in neutral PDRs, the harder radiation field at lower metallicities will produce hard photons capable of ionizing $\mathrm{O}^{+}(\mathrm{IP}=35.1 \mathrm{eV})$. Since the ionization potential of $\mathrm{C}^{+}$is only $24.4 \mathrm{eV}$, carbon might thus easily become doubly ionized
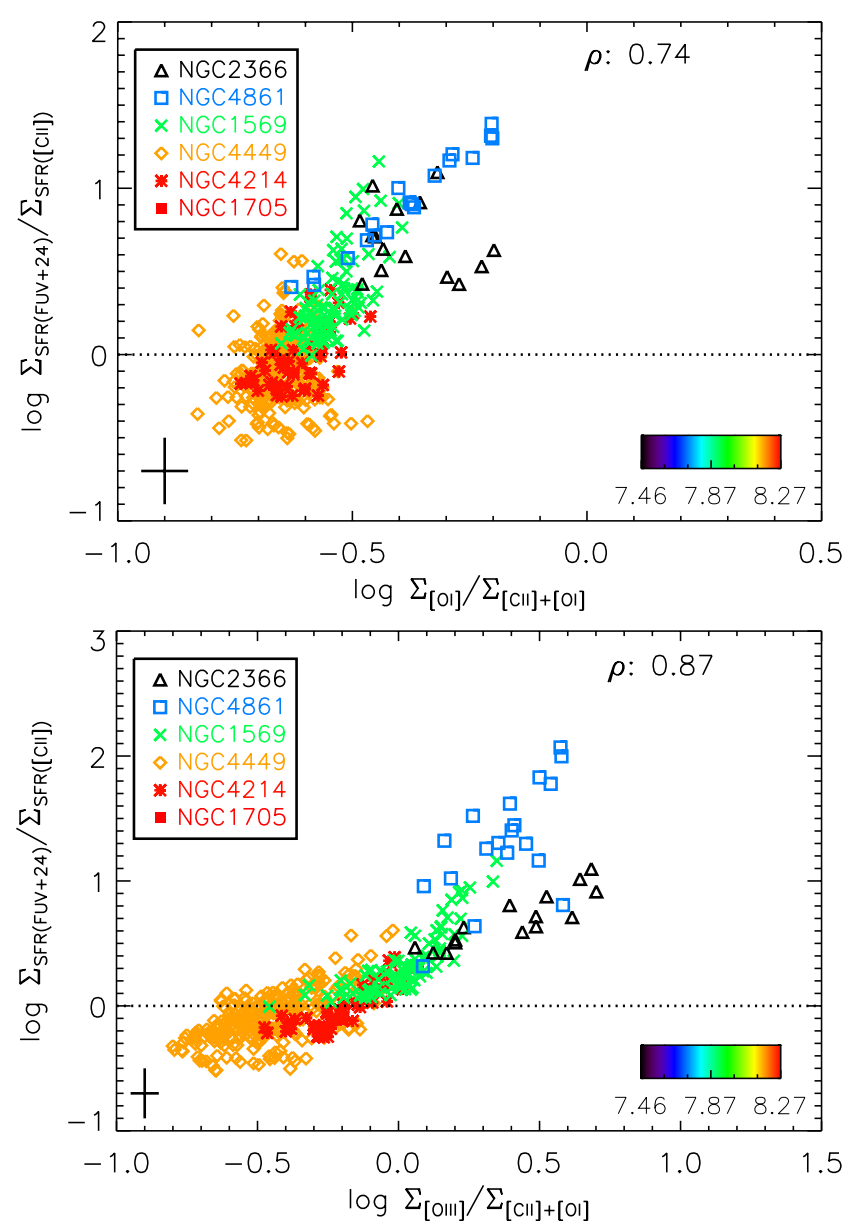

Fig. 5. Spatially resolved galaxy relation between the dispersion from the SFR calibrations for $[\mathrm{CII}]$ as a function of $[\mathrm{OI}]_{63} /[\mathrm{CII}]+[\mathrm{OI}]_{63}($ top $)$ and $[\mathrm{OIII}]_{88} /[\mathrm{CII}]+[\mathrm{OI}]_{63}($ bottom $)$ line ratios. The image format is the same as explained in Fig. 4.

in diffuse ionized gas media resulting in most of the carbon being locked in $\mathrm{C}^{++}$rather than $\mathrm{C}^{+}$. In particular, metal-poor regions seem affected by large filling factors of highly ionized media, casting doubt on the ability of [CII] to trace the SFR in those environments.

\section{Global galaxy SFR- $L_{\text {line }}$ relation}

In the previous section, we analyzed the observed trends and scatter in the $S F R-L_{\text {line }}$ relations for a subsample of spatially resolved galaxies down to hectoparsec scales. We now verify whether the trends and scatter remain present on global galaxy scales, when averaged out over the different ISM phases. For this analysis, we consider all galaxies with GALEX FUV and MIPS $24 \mu \mathrm{m}$ observations (32 out of 48 galaxies), including the resolved sources from Sect. 3 with their line and continuum flux measurements reduced to one data point. The extension of the subsample of spatially resolved galaxies to the entire DGS sample broadens the range covered in metallicity from $0.03 \mathrm{Z}_{\odot}$ (IZw 18) to $0.5 Z_{\odot}$ (HS 2352+2733) and in SFR from $0.001 M_{\odot} \mathrm{yr}^{-1}$ (UGC 4483 ) to $43 M_{\odot} \mathrm{yr}^{-1}$ (Haro 11) as traced by $\mathrm{FUV}+24 \mu \mathrm{m}$.

\subsection{Global fluxes}

For global galaxy fluxes of FIR lines, we rely on the aperture photometry results for fine-structure lines reported in 
Cormier et al. (2012), where FIR line fluxes within apertures covering the brightest fine-structure line emission are computed. We assume a $30 \%$ calibration error on top of the uncertainties that result from line fitting. In some cases, the [CII] emission is more extended with respect to the $[\mathrm{OI}]_{63}$ and $[\mathrm{OIII}]_{88}$ emission or is simply observed across a wider field, in which case the [CII] apertures are bigger to include the total region mapped. Here, we measure the $[\mathrm{CII}]$ flux within the same apertures as the $[\mathrm{OI}]_{63}$ and $[\mathrm{OIII}]_{88}$ emission for six galaxies using the same techniques as Cormier et al. (2012). For NGC 6822, we only include the FIR line measurements from the HII region Hubble V, since it is the only area in NGC 6822 covered in all three lines.

Corresponding GALEX FUV and MIPS $24 \mu \mathrm{m}$ fluxes are obtained from aperture photometry using the central positions and aperture sizes applied to the FIR fine-structure lines. Table B.1 gives an overview of the aperture photometry results for GALEX FUV and MIPS $24 \mu \mathrm{m}$ bands. For point sources, GALEX FUV and MIPS $24 \mu \mathrm{m}$ measurements usually correspond to total galaxy fluxes. Total MIPS $24 \mu \mathrm{m}$ fluxes for point sources are adopted from Bendo et al. (2012b) and are indicated with an asterisk in Col. 5 of Table B.1. In some cases, the FUV data show an extended tail of emission (HS 1442+4250, UGC 04483, UM 133) with no counterpart in MIPS $24 \mu \mathrm{m}$ nor PACS maps. Rather than measuring the global FUV emission for these galaxies with a cometary structure, we rely on aperture photometry within apertures that encompass the brightest $24 \mu \mathrm{m}$ emission features. In this manner, we avoid overestimating the total SFR for these galaxies by neglecting the FUV emission that was either not covered in our Herschel observations or did not show any dust emission, suggesting that little dust is present in those areas. Table B.1 reports the FUV flux densities within those apertures, but also provides in parentheses the photometry results for apertures encompassing the total FUV emission.

\subsection{Observed trends}

Figure 6 presents the $S F R-L_{\text {line }}$ relations on global galaxy scales for DGS sources with GALEX FUV and MIPS $24 \mu \mathrm{m}$ observations. Based on the observed trends, SFR calibrations are derived from linear regression fits:

$\log S F R=\beta+\alpha * \log L_{\text {line }}$.

where $L_{\text {line }}$ is the FIR line luminosity in units of $L_{\odot}$, SFR is the star formation rate in units of $M_{\odot} \mathrm{yr}^{-1}$, and $\alpha$ and $\beta$ represent the slope and intercept of the best fit. Table 2 (see bottom part) summarizes the calibration coefficients (slope, intercept) retrieved from the fits and the dispersion of data points around the best fit.

With the slopes of all best-fit lines determined with at least $5 \sigma$ significance, we are confident that the SFR also correlates with the $[\mathrm{CII}],[\mathrm{OI}]_{63}$, and $[\mathrm{OIII}]_{88}$ line emission on global scales. The smallest dispersion $(0.25 \mathrm{dex})$ and strongest constraint on the slope $(S / N \sim 19)$ could be obtained for the $[\mathrm{OI}]_{63}$ line, from which the SFR can be estimated with an uncertainty factor of 1.8. The [OIII $]_{88}$ line probes the SFR within an uncertainty factor of 2, while the link between the SFR and [CII] line is more dispersed and results in an estimated SFR uncertain by a factor of $\sim 2.4$. The top panel of Figure 6 includes previous SFR calibrations reported in De Looze et al. $(2011)^{5}$ (dashed red line)

\footnotetext{
5 The SFR calibration in De Looze et al. (2011) was derived based on the reference SFR tracers GALEX FUV and MIPS $24 \mu \mathrm{m}$ and the scaling factor $\alpha=6.31$, as derived by Zhu et al. (2008). Recalibrating their relation with the scaling factor $(\alpha=3.89)$ applied in this paper would only shift their relation by 0.2 dex at most.
}
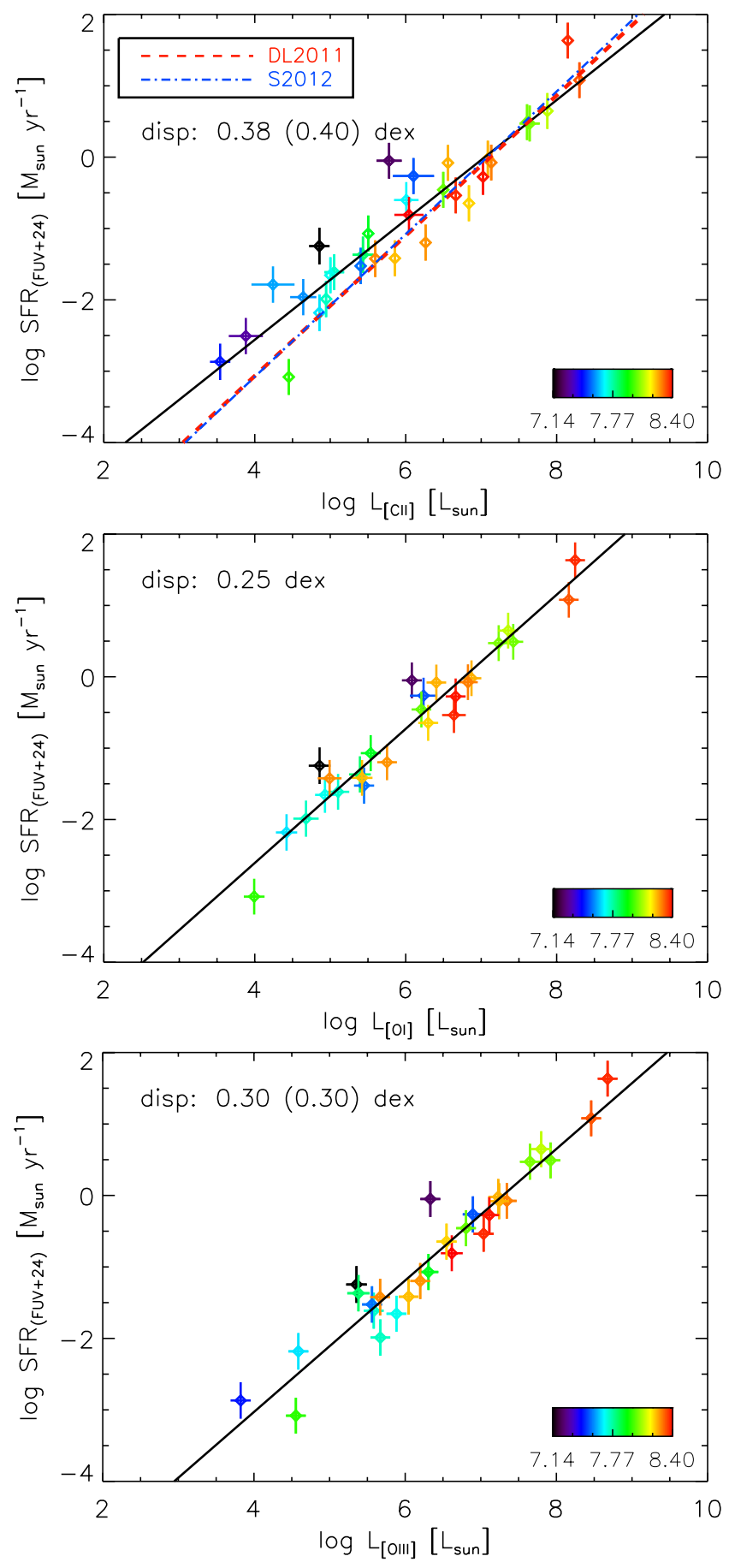

Fig. 6. Relation between the SFR and [CII] (top), $[\mathrm{OI}]_{63}$ (middle), and $[\mathrm{OIII}]_{88}$ (bottom) luminosities on global galaxy scales. Galaxies are color-coded according to metallicity with increasing oxygen abundances ranging from black then blue, green and yellow to red colors. The best-fit SFR calibration is presented as a solid, black line. The dispersion of data points around the SFR calibration is indicated at the top of each panel, with the number in parentheses indicating the scatter for the complete sample with $>5 \sigma$ detections for all three lines.

and Sargsyan et al. (2012) (blue dashed-dotted line). The SFR$L_{[\mathrm{CII}]}$ calibration derived for the DGS sample ${ }^{6}$ has a shallower

6 Although the [CII] luminosity range in De Looze et al. (2011) $(5.7 \leq$ $\left.\log L_{\text {[CII] }}\left[L_{\odot}\right] \leq 9.1\right)$ does not extend to the faintest [CII] luminosities for galaxies, it largely overlaps with the $L_{[\mathrm{CII}]}$ range covered by DGS sources while the SFR relation in Sargsyan et al. (2012) was calibrated for higher [CII] luminosities $\left(7 \leq \log L_{[\mathrm{CII}]}\left[L_{\odot}\right] \leq 9\right)$. 
slope ( $\alpha=0.84$ ) compared to the nearly one-to-one correlation obtained in De Looze et al. (2011) and Sargsyan et al. (2012), which can be attributed to a decreasing [CII] emission toward lower metal abundances. For $[\mathrm{CII}]$ and $[\mathrm{OIII}]_{88}$, the SFR seems qualitatively linked in the same way to the line emission on global galaxy and spatially resolved scales. On spatially resolved scales, the slope of the SFR calibrations for $[\mathrm{OI}]_{63}(\alpha=1.41)$ differs from the correlation observed on global galaxy scales ( $\alpha=0.94)$.

Compared to the dispersion in the spatially resolved $S F R-L_{\text {line }}$ relations, the averaging over the different ISM phases on global galaxy scales does not reduce the scatter in the observed $S F R-L_{\text {line }}$ trends. This again shows that the dispersion in the SFR relations is not driven by variations within one single galaxy, but rather originates from the diversity of ISM conditions in a large sample of galaxies covering wide ranges in metallicity. The dispersion is largest in the $S F R-L_{[\mathrm{CII}]}$ trend $(0.38 \mathrm{dex})$ as compared to the $S F R-L_{[\mathrm{OIII}]}(0.30 \mathrm{dex})$ and $S F R-L_{[\mathrm{OI}]}(0.25 \mathrm{dex})$ relations. For a sample of similar size (24 galaxies), the SFR calibration for [CII] presented in De Looze et al. (2011) reports a $1 \sigma$ dispersion of only 0.27 dex. Part of the increased scatter observed for the DGS sample might be attributed to the uncertainties on the reference SFR tracer, which was shown to be sensitive to the star formation history and, possibly, the grain properties of metal-poor dwarf galaxies (see Sects. A.2 and A.3). We argue, however, that the significant scatter in the $S F R-L_{[\mathrm{CII}]}$ relation indicates a large variety of ISM conditions (i.e., gas density, radiation field, filling factors of neutral and ionized gas, excitation conditions) probed in the DGS galaxy sample (see Sect. 4.3). This diversity might not be surprising given the different morphological classifications (e.g. blue compact dwarfs, low-surface brightness objects, luminous infrared galaxies, interacting galaxies) of the dwarf galaxies in the DGS sample.

On global galaxy scales, the $[\mathrm{OI}]_{63}$ line is considered a better intrinsic tracer of the SFR for the DGS sample compared to $[\mathrm{CII}]$ and $[\mathrm{OIII}]_{88}$, which suggests that the fraction of gas heating in warm and/or dense PDRs is a good approximation of the level of star formation activity across a wide range of metallicities. We caution that the $[\mathrm{OI}]_{63}$ emission in extremely metal deficient objects is not necessarily linked to the classical PDRs, but might rather be powered by soft X-rays (e.g. Péquignot 2008; Lebouteiller et al., in prep.).

To better approximate the overall gas cooling budget in galaxies, and, hereby, the heating through star formation under the assumption of local thermal equilibrium, we attempt to fit SFR calibrations with different combinations of FIR lines of the form:

$\log S F R=\beta+\log \left(L_{[\mathrm{CII}]}^{\alpha_{1}}+L_{[\mathrm{OI}]}^{\alpha_{2}}+L_{[\mathrm{OIII}]}^{\alpha_{3}}\right)$.

By combining the emission of two or three FIR lines (CII], $[\mathrm{OI}]_{63}$, and $\left.[\mathrm{OIII}]_{88}\right)$, we do not improve on the scatter in the SFR calibrations on global galaxy scales. The combination of the brightest FIR lines on spatially resolved scales of about $100 \mathrm{pc}$ did diminish the scatter in the $S F R-L_{\text {line }}$ relations, suggesting that other cooling lines (potentially in the optical wavelength domain) and/or gas heating mechanisms (unrelated to star formation) become important on global galaxy scales.

\subsection{Scatter in the $S F R-L_{\text {line }}$ relation}

In this section, we focus on identifying the parameters that drive the dispersion in the $S F R-L_{\text {line }}$ relations. Figures 7-9 show the observed trends between the scatter in the $S F R-L_{\text {line }}$ relations
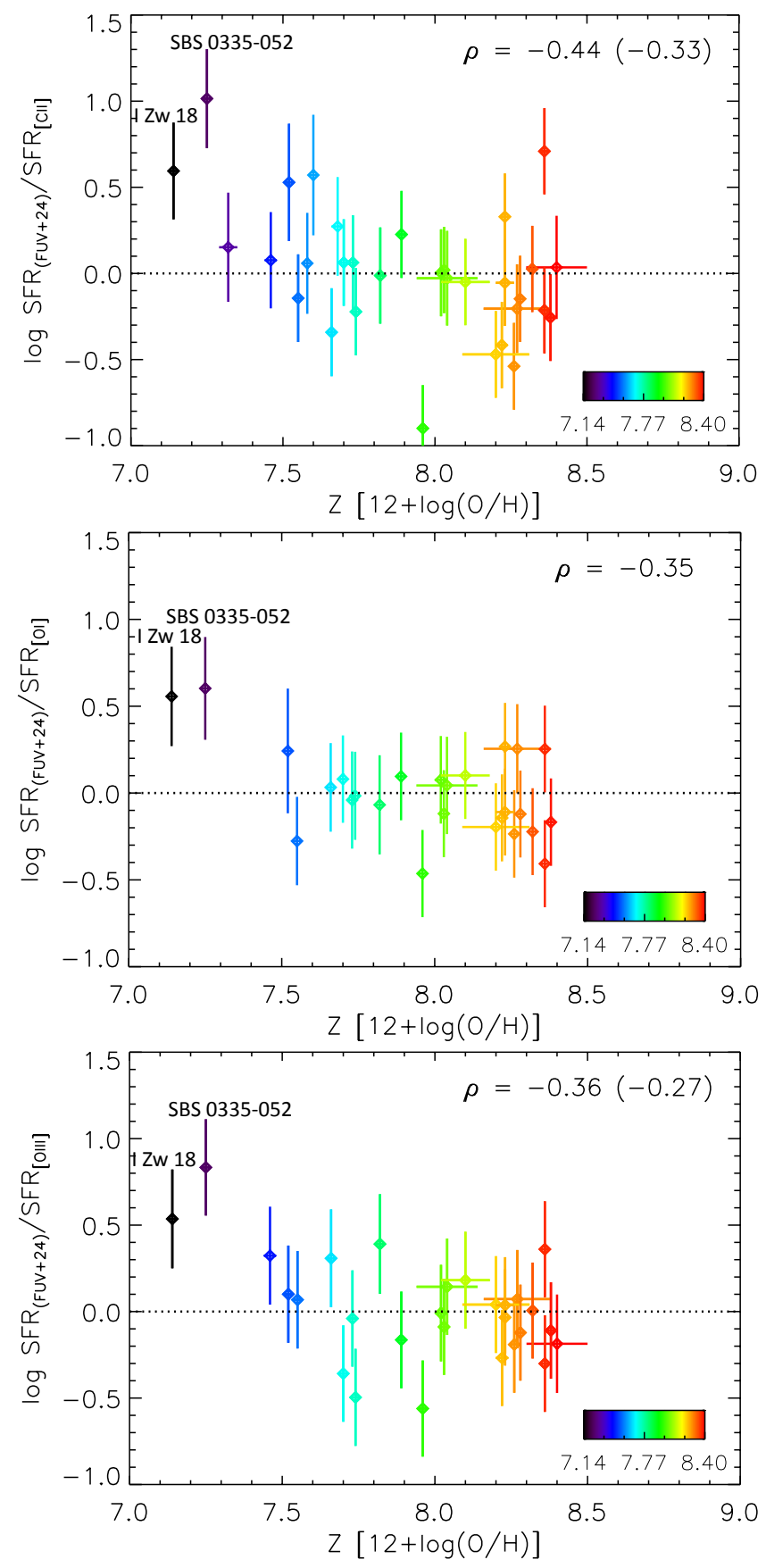

Fig. 7. Relation between the dispersion from the SFR calibrations for [CII] (top), [OI $]_{63}$ (middle), [OIII $]_{88}$ (bottom) as a function of oxygen abundance, $12+\log (\mathrm{O} / \mathrm{H})$, on global galaxy scales. Galaxies are color-coded according to metallicity with increasing oxygen abundances ranging from black then blue, green and yellow to red colors. The Spearman's rank correlation coefficients are indicated in the top right corner. In parentheses, we show the dispersion for the complete galaxy sample, i.e., global galaxies that have $>5 \sigma$ detections for all three lines $[\mathrm{CII}],[\mathrm{OI}]_{63}$, and $[\mathrm{OIII}]_{88}$.

and the metal abundance, dust temperature, and ISM structure as probed through the line ratios $[\mathrm{OI}]_{63} /[\mathrm{CII}]+[\mathrm{OI}]_{63}$ and $[\mathrm{OIII}]_{88} /[\mathrm{CII}]+[\mathrm{OI}]_{63}$, respectively. The dust temperatures of 25 out of 32 galaxies are constrained by the results from a modified blackbody fitting routine with variable dust emissivity index $\beta$ presented in Rémy-Ruyer et al. (2013).

The shallow slope in the SFR calibrations for the DGS sample (as compared to the slopes for the literature sample in 
Ilse De Looze et al.: The applicability of far-infrared fine-structure lines as star formation rate tracers
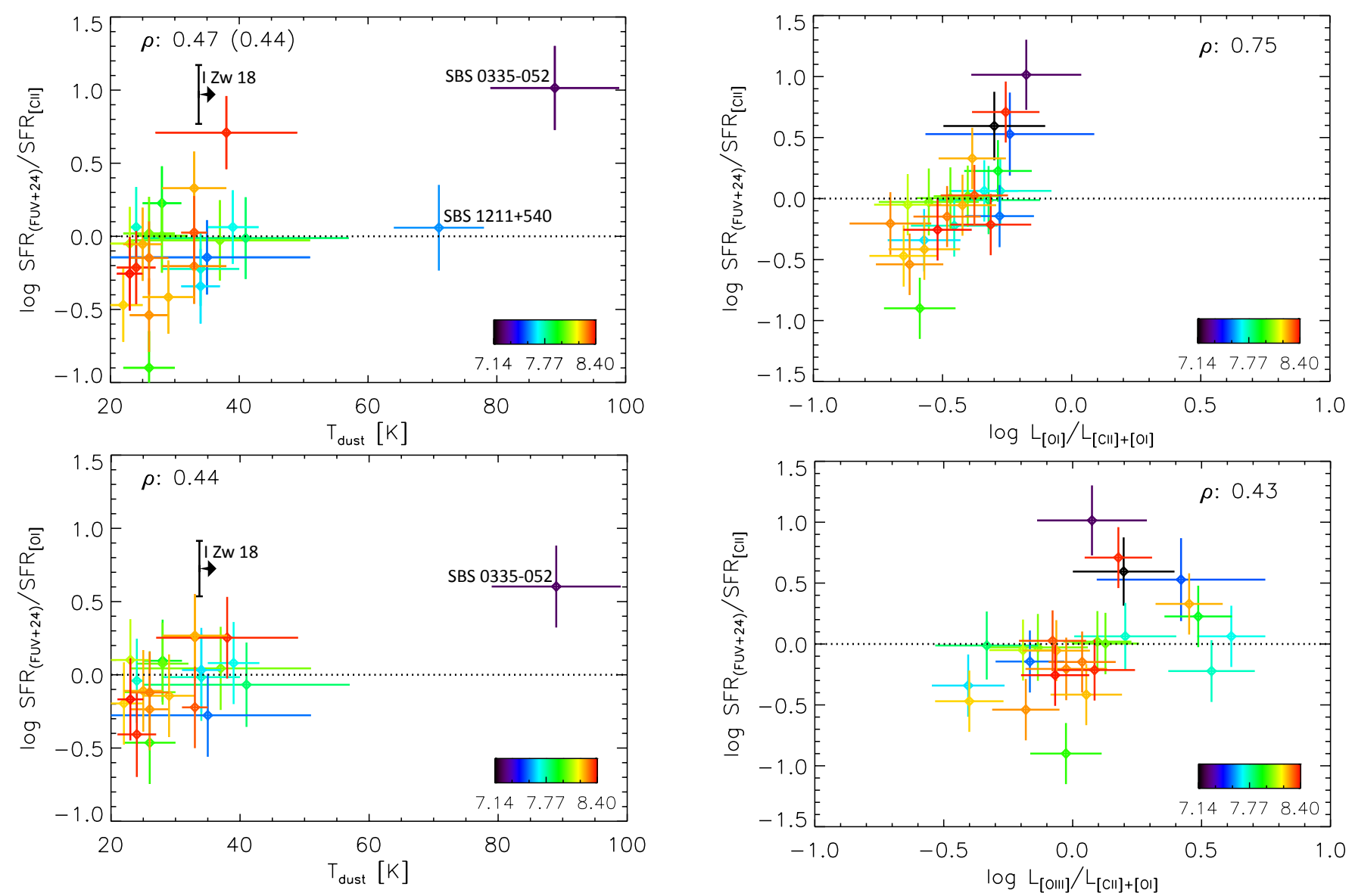

Fig. 8. Relation between the dispersion from the SFR calibrations for [CII] (top), and [OI $]_{63}$ (bottom) as a function of dust temperature, $T_{\text {dust }}$, on global galaxy scales. The galaxy IZw 18 was not detected at PACS $160 \mu \mathrm{m}$ wavelengths and, therefore, the fitting procedure was not attempted in Rémy-Ruyer et al. (2013). Herrera-Camus et al. (2012) estimated a lower limit for the dust temperature $T_{\mathrm{d}} \geq 33.7 \mathrm{~K}$ based on the PACS $70 \mu \mathrm{m}$ flux and PACS $160 \mu \mathrm{m}$ upper limit, which is used to indicate the position of IZw 18 in the plots of Fig. 4. The image format is the same as explained in Fig. 7.

Sect. 5) and the weak correlations in Fig. $7(\rho=-0.44$ for [CII], $\rho=-0.35$ for $[\mathrm{OI}]_{63}$, and $\rho=-0.36$ for $[\mathrm{OIII}]_{88}$ ) suggest that the metal abundance has an effect on the quantitative link between the SFR and FIR line emission (in particular for [CII]). The true offset of the lowest abundance dwarfs might be even higher because of an underestimation of their SFR based on FUV emission (see discussion in Sect. A.2). The weaker [CII] emission toward lower metal abundances is consistent with the drop in [CII] surface brightness in PDR models by a factor of about 5 from metallicities of $12+\log (\mathrm{O} / \mathrm{H}) \sim 8.5$ down to $12+\log (\mathrm{O} / \mathrm{H}) \sim 7.5$ (Röllig et al. 2006), (i.e., drop of a factor of 10 in metallicity) for a single cloud with density $n_{\mathrm{H}} \sim 10^{3} \mathrm{~cm}^{-3}$ (see their Fig. 6). The weak trends for $[\mathrm{OI}]_{63}$ and $[\mathrm{OIII}]_{88}$ are mainly driven by two galaxies of extreme low metal abundance, I Zw 18 and SBS 0335-052, in which the [OI $]_{63}$ and [OIII $]_{88}$ lines do not seem to add significantly to the overall gas cooling. Since the SFR is unlikely to be overestimated for these galaxies based on FUV+MIPS $24 \mu \mathrm{m}$ (see comparison with other SFR tracers in Sect. A of the Appendix), the offset of these extremely dust deficient galaxies might suggest that other lines dominate the cooling processes (e.g., Ly $\alpha$ ). The gas heating might, alternatively, be dominated by heating mechanisms other than the photoelectric effect (e.g., soft X-ray heating, mechanical heating, cosmic

Fig. 9. Relation between the dispersion from the SFR calibrations for $[\mathrm{CII}]$ as a function of $[\mathrm{OI}] /[\mathrm{CII}]+[\mathrm{OI}]_{63}($ top $)$ and $[\mathrm{OIII}]_{88} /[\mathrm{CII}]+[\mathrm{OI}]_{63}$ (bottom) line ratios, on global galaxy scales. The image format is the same as explained in Fig. 7.

rays), which could disperse the link between the emission of cooling lines and the SFR. For IZw 18, the heating has indeed been shown to be dominated by soft X-ray heating (Péquignot 2008; Lebouteiller et al., in prep.), which is likely to also affect the $S F R-L_{\text {line }}$ relations.

The dust temperatures of galaxies seem to correlate (weakly) with the dispersion in the SFR calibration for [CII] $(\rho=0.47)$ and $[\mathrm{OI}]_{63}(\rho=0.44)$. The global galaxy analysis, hereby, confirms the trends observed in Fig. 4 on spatially resolved galaxy scales. With the DGS sources showing a trend of increasing dust temperature with decreasing metal abundance (Rémy-Ruyer et al. $2013)$, the correlation of the dispersion in the $S F R-L_{[\mathrm{CII}]}$ and $S F R-L_{[\mathrm{OI}]}$ relations with dust temperature seems, at least in part, to be related to the metallicity of DGS sources.

The dispersion in the $S F R-L_{[\mathrm{CII}]}$ relation clearly correlates with the $[\mathrm{OI}]_{63} /[\mathrm{CII}]+[\mathrm{OI}]_{63}$ line ratio $(\rho=0.75)$, while a trend is also present, although less obvious, for $[\mathrm{OIII}]_{88} /[\mathrm{CII}]+[\mathrm{OI}]_{63}$ $(\rho=0.43)$. Making similar plots for the dispersion in the SFR relation for $[\mathrm{OI}]_{63}$ as a function of $[\mathrm{OI}]_{63} /[\mathrm{CII}]+[\mathrm{OI}]_{63}(\rho=0.35)$ does not reveal a clear trend (graph is not shown here), suggesting that the $[\mathrm{OI}]_{63}$ line is capable of tracing the SFR in a consistent way irrespective of changes in the ISM structure.

To understand the observed trends between the scatter in the $S F R-L_{\text {line }}$ relations and several physical parameters, we try to link the low abundance to the warm dust temperatures and different ISM structure observed in low-metallicity galaxies. In low-abundance galaxies, the fraction of metals is lower in the 
solid as well as gas phase. The lower abundance of grains, however, does not directly cause a decrease in the photoelectric heating efficiency, since it is, at least partially, compensated by a higher heating rate in dwarf galaxies, exhibited by their hotter average temperature (e.g., Rémy-Ruyer et al. 2013). Similarly, the lower abundances of $\mathrm{C}$ and $\mathrm{O}$ in the gas phase will be balanced by higher line cooling rates. Deficits of species like $\mathrm{C}$ and $\mathrm{N}$ could, however, occur relative to the $\mathrm{O} / \mathrm{H}$ abundance which is used here to measure metallicity via the relative $\mathrm{O}$ abundance. This could result in relatively less cooling provided by the $\mathrm{C}$ lines.

The photon escape fraction might become more important with decreasing metallicity due to the porosity of the ISM, which lowers the energy input for the heating of dust and gas through the photoelectric effect. Other than higher photon escape fractions, the hard radiation fields in low-metallicity environments can also enhance grain charging, making grains less efficient for the photoelectric effect (e.g., Tielens \& Hollenbach 1985a; Malhotra et al. 1997; Negishi et al. 2001; Croxall et al. 2012; Farrah et al. 2013).

Indeed, stars at lower metallicities have higher effective temperatures due to line blanketing effects. For a given stellar age and mass, they will produce more hard photons than at solar luminosities. Because of the longer mean free path lengths of UV photons in dust deficient media, the ionization of gas and participation in the gas/dust heating persist over large distances from the ionizing sources in star-forming complexes, which furthermore increases the dust temperatures. As a consequence of the longer distances traversed by ionizing photons, the $\mathrm{C}^{+}$-emitting zone in galaxies can be enlarged compared to higher metallicity environments due to the deeper penetration of FUV photons capable of photodissociating CO molecules (e.g., Poglitsch et al. 1995; Israel et al. 1996; Madden et al. 1997; Israel \& Maloney 2011). Also, the filling factor of ionized gas phases will enlarge due to the hardness of the radiation field and the transparency of the ISM in low-metallicity objects.

Grain properties might, furthermore, vary in objects of lower metal abundance. The PAH emission has been shown to decrease below $12+\log (\mathrm{O} / \mathrm{H}) \sim 8.1$ (Boselli et al. 2004; Engelbracht et al. 2005; Jackson et el. 2006; Madden et al. 2006; Draine et al. 2007; Engelbracht et al. 2008; Galliano et al. 2008), while the abundance of very small grains grows drastically relative to the large grain population due to the fragmentation of those large grains through shocks experienced in the turbulent ISM (Jones et al. 1996; Lisenfeld et al. 2002; Galliano et al. 2003, 2005). Knowing that PAHs and very small grains are the main contributors to the photoelectric effect, the outcome on the gas heating efficiency and the subsequent gas cooling remains a puzzle.

In summary, we argue that the best SFR tracer varies for different environments depending on the density and ionization state of the gas. Due to the hardness of the radiation field and longer mean free path lengths in metal-poor galaxies, the filling factors of ionized gas media are bound to grow drastically, resulting in $[\mathrm{CII}]$ and $[\mathrm{OI}]_{63}$ being poor SFR diagnostics. In such highly ionized regions, we expect most of the carbon and oxygen to be locked in elements with higher ionization potentials. The $[\mathrm{CII}]$ and $[\mathrm{OI}]_{63}$ line emission might, furthermore, be affected by a decrease in the photoelectric efficiency due to higher photon escape fractions and/or grain charging. The reliability of $[\mathrm{OIII}]_{88}$ as an SFR indicator mainly relies on the filling factor of diffuse, highly ionized gas. The large range covered in $[\mathrm{OIII}]_{88} /[\mathrm{CII}]+[\mathrm{OI}]_{63}$ (from -0.4 to 0.6 ) suggests that the relative filling factors of PDRs and ionized media can differ significantly from one galaxy to another, depending on the hardness of the radiation field, excitation conditions, and the filling factor of low-density gas relative to compact gas clumps. The choice of a reference SFR tracer would, thus, benefit from knowledge of the ionization state and density of the gas. Without any precursory constraints on the ISM conditions, the $[\mathrm{OI}]_{63}$ line is considered to be the most reliable SFR indicator for galaxies covering a wide range in metal abundances.

\section{Prescriptions for extending the SFR calibrations to other galaxy samples}

In this section, we derive SFR calibrations for different galaxy populations. Hereto, we gather FIR fine-structure line measurements from the literature for dwarf galaxies, starbursts, ULIRGs, galaxies harboring an AGN, and high-redshift galaxies (ranging from $z=0.5$ to 6.6). The entire galaxy sample constitutes of 530, 150 , and 102 galaxies with $[\mathrm{CII}],[\mathrm{OI}]_{63}$, and [OIII $]_{88}$ detections, respectively.

\subsection{Literature sample}

The literature sample of the local Universe $(z<0.5)$ was assembled from FIR line measurements published based on ISO observations in Brauher et al. (2008, 83 galaxies) and Herschel data in Parkin et al. (2013, 1 galaxy), Sargsyan et al. (2012, 101 galaxies), Díaz-Santos et al. (2013, 206 galaxies), Farrah et al. (2013, 24 galaxies), Graciá-Carpio et al. (2011, in prep., 56 galaxies). Where duplications exist between ISO and Herschel spectroscopy, we choose the Herschel data (see Sect. A.4 for a comparison between Herschel and ISO spectroscopy measurements.). For the Brauher et al. (2008) sample of ISO observations, we consider all galaxies with emission unresolved with respect to the ISO beam. While De Looze et al. (2011) only considered galaxies with GALEX FUV and MIPS $24 \mu \mathrm{m}$ observations, we extend the literature sample to 84 galaxies from the Brauher et al. (2008) sample with IRAS 12, 25, 60, and $100 \mu \mathrm{m}$ flux measurements from which the TIR luminosity and, thus, the SFR can be computed. Although some of the galaxies from the Brauher et al. (2008) sample have been observed with Herschel, the lack of their published FIR line fluxes led to the usage of the ISO flux measurements. All the literature works of Herschel observations present the FIR line measurements of all three lines of interest ([CII], $[\mathrm{OI}]_{63}$, [OIII $]_{88}$ ), with the exception of Sargsyan et al. (2012) and Díaz-Santos et al. (2013) ${ }^{7}$ reporting only [CII] measurements. The Great Observatories All-sky LIRG Survey (GOALS) sample (Díaz-Santos et al. 2013) was complemented with data from other Herschel programs. We exclude sources already presented in Sargsyan et al. (2012) and Graciá-Carpio et al. (in prep.) (and, thus, already part of our literature sample), resulting in 206 sources of the original GOALS sample.

FIR line detections and upper limits for high-redshift galaxies $^{8}$ are gathered for fine-structure lines [CII], [OI $]_{63}$, and $[\mathrm{OIII}]_{88}$ based on observations with a wide variety of groundbased facilities and the Herschel Space Observatory. To convert redshifts to luminosity distances, we use the NED cosmology calculator (Wright 2006), assuming a spatially flat cosmology with $H_{0}=67.3 \mathrm{~km} \mathrm{~s}^{-1} \mathrm{Mpc}^{-1}, \Omega_{\lambda}=0.685$ and $\Omega_{\mathrm{m}}=0.315$ (Planck Collaboration XVI 2014).

\footnotetext{
Other FIR lines have been observed for the GOALS sample, but have not yet been published.

8 We refer to high-redshift galaxies starting from redshifts $z \geq 0.5$.
} 


\subsection{Source classification}

We classify galaxies as dwarfs if the criterion $L_{\mathrm{H}}<10^{9.6} L_{\mathrm{H}, \odot}$ is fulfilled, similar to the selection procedure applied in Boselli et al. (2008). We do not distinguish between the different classifications of dwarf galaxies (e.g., blue compact dwarfs, late-type spirals, Magellanic irregulars).

For the more massive galaxy populations, we make a distinction between the dominant power source for infrared emission, i.e., star formation or AGN activity. To homogenize the classification of starburst, composite, and AGN sources for the different literature datasets, we adapt the source classification of Sargsyan et al. (2012) to the selection criteria used in DíazSantos et al. (2013) based on the equivalent width (EW) of the mid-infrared PAH feature at $6.2 \mu \mathrm{m}$. More specifically, galaxies are considered to be AGN-dominated if $E W(\mathrm{PAH} 6.2 \mu \mathrm{m})$ $\lesssim 0.3$ and classified as pure starburst if $E W(\mathrm{PAH} 6.2 \mu \mathrm{m}) \gtrsim 0.5$. Galaxies characterized by intermediate equivalent width values are considered composite sources, i.e., with starburst and AGN contributions to the mid-infrared features. Applying these selection criteria results in the classification of 94 composite/AGN sources and seven starburst galaxies from the galaxy sample presented in Sargsyan et al. (2012), among which 19 can be classified as ULIRGs. The GOALS sample consists of 129 starburst galaxies and 77 AGN or composite sources, among which two can be assigned ULIRGs. Based on the optical source classification of Farrah et al. (2013), we identify six HII-dominated/starburst galaxies and 18 LINER/Seyfert galaxies. For the Brauher et al. (2008) sample, we use an optical classification similar to De Looze et al. (2011) to distinguish between purely star-forming objects $(37 \mathrm{HII} / \mathrm{starburst}$ galaxies) and objects with power sources other than star formation (36 transition/LINER/Seyfert galaxies). Galaxies with no or uncertain object classifications in NED were omitted from our sample. The Brauher et al. (2008) sample, furthermore, includes ten dwarf galaxies. Based on the optical source classification for the SHINING sample (Survey with Herschel of the Interstellar medium in Nearby infrared Galaxies, Fischer et al. 2010; Sturm et al. 2010; Graciá-Carpio et al. 2011, in prep.), we identify 20 starburst galaxies and 36 composite or AGN sources, among which 21 objects fulfill the criterion for ULIRGs $\left(L_{\mathrm{IR}}>10^{12} L_{\odot}\right)$. We classify the central region of M 51 as HIIdominated, since Parkin et al. (2013) argue that the AGN in M 51 does not significantly affect the excitation of gas.

To verify that the optical source classification is consistent with the classification based on the EW of the mid-infrared PAH feature at $6.2 \mu \mathrm{m}$, we compare the results for a subsample of 19 galaxies from the SHINING sample with measurements of EW (PAH 6.2 $\mu \mathrm{m}$ ) reported in Stierwalt et al. (2013) for all objects of the GOALS sample. The optical classification coincides with the limits in EW (PAH $6.2 \mu \mathrm{m}$ ) to distinguish between pure starbursts and composite/AGN sources, with the exception of five ULIRGs. The high level of obscuration in ULIRGs impedes the classification, but since we treat ULIRGs as a separate population with $L_{\mathrm{IR}}>10^{12} L_{\odot}$ distinct from starburst and composite/AGN galaxies with lower infrared luminosities, we are confident that the different methods applied for the source classification are consistent for galaxies with $L_{\mathrm{IR}}<10^{12} L_{\odot}$.

\subsection{Reference SFR calibrator}

For dwarf galaxies, we estimate the SFR from the same combination of (un)obscured SFR tracers (GALEX FUV) used for the DGS sample (see Sect. 2.3). We use the GALEX FUV and MIPS
$24 \mu \mathrm{m}$ flux measurements reported in De Looze et al. (2011), when available. For the remaining sources, we retrieve GALEX FUV fluxes from the GALEX catalog ${ }^{9}$. Catalog FUV measurements have been corrected for Galactic extinction according to the recalibrated $A_{V}$ in Schlafly et al. (2011) from Schlegel et al. (1998), as reported on NED, and assuming an extinction law with $R_{V}=3.1$ derived in Fitzpatrick et al. (1999). Relying on the conclusions drawn in Kennicutt et al. (2009) for the Spitzer Infrared Nearby Galaxies Survey (SINGS), we assume that the emission from MIPS $24 \mu \mathrm{m}$ and IRAS $25 \mu \mathrm{m}$ can be used interchangeably. We collect IRAS $25 \mu \mathrm{m}$ flux densities from the IRAS Revised Bright Galaxy Sample (Sanders et al. 2003) or, alternatively, from the IRAS Faint Source Catalog (Moshir \& et al. 1990).

For all other galaxies in the literature sample, we estimate the star formation rates based on the TIR luminosity $\left(L_{\mathrm{TIR}}{ }^{10}\right.$, $8-1000 \mu \mathrm{m})$ and the SFR calibration reported in Hao et al. (2011); Murphy et al. (2011) (see also Table A.1). Total infrared luminosities are reported in Sargsyan et al. (2012) and Farrah et al. (2013). For the SHINING sample, we compute FIR (42.5-122.5 $\mu \mathrm{m}$ ) luminosities from the Herschel continuum flux densities at 63 and $122 \mu \mathrm{m}$, which could be determined based on a proper continuum estimation from the $[\mathrm{OI}]_{63}$ and $[\mathrm{NII}]_{122}$ line observations (see Graciá-Carpio et al. in prep.). Constraining the FIR luminosities in this manner (rather than relying on the total IRAS flux densities to compute FIR) allows us to determine the infrared emission within the same regions as the PACS line observations, preventing any overestimation of the SFR for galaxies only partly covered by Herschel spectroscopy observations. For the Brauher et al. (2008) sample, we use the IRAS flux densities at $12,25,60$, and $100 \mu \mathrm{m}$ to compute the TIR luminosity based on the formulas from Sanders \& Mirabel (1996). Similarly, the IRAS 60 and $100 \mu \mathrm{m}$ flux densities are used to compute $L_{\mathrm{FIR}}$ and converted to $L_{\mathrm{TIR}}$ using a correction factor of 1.75 for the GOALS sample. The SFR in M 51 is estimated from the total infrared luminosity in the central $80^{\prime \prime}$ region, reported in Parkin et al. (2013).

Table B.2 summarizes the FIR line measurements obtained from the literature and quotes $L_{\mathrm{TIR}}$ and SFR of high-redshift sources derived in this manner. Since the uncertainties in the line and FIR luminosities (and thus SFR estimates) for high-redshift galaxies depend strongly on the uncertainty of their assumed distance, relying on a specific cosmological model with underlying uncertainties, as well as a possible magnification factor for lensed sources, we safely assume a conservative uncertainty of $50 \%$ in both the line luminosity and SFR estimate.

Tables B.3-B.5 give an overview of the galaxies classified as dwarfs, HII/starburst, and AGN, respectively, and indicate their name, luminosity distance $D_{L}$, reference for their FIR line measurements, total infrared luminosity $(8-1000 \mu \mathrm{m})$, and SFR. In both tables, ULIRGs are indicated with an asterisk behind their name.

\footnotetext{
9 http://galex.stsci.edu/GR6/

10 Often FIR luminosities (42.5-122.5 $\mu \mathrm{m}$ ) are reported, while the SFR calibration requires the total infrared luminosity, $L_{\mathrm{TIR}}$ (see Table A.1). We use a common conversion factor of 1.75 to translate the quoted FIR into total infrared luminosities, following Calzetti et al. (2000). Some authors apply the convention that $L_{\mathrm{FIR}}$ is the luminosity in the wavelength range $40-500 \mu \mathrm{m}$. We convert the latter FIR luminosities to $L_{\mathrm{TIR}}$ using a conversion factor of 1.167 , following the factor of 1.75 (Calzetti et al. 2000) to convert from FIR $(42.5-122.5 \mu \mathrm{m})$ to TIR $(8-1000 \mu \mathrm{m})$

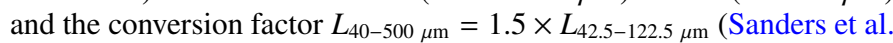
2003).
} 
Table 3. Prescriptions to estimate the SFR from the relation $\log S F R\left[M_{\odot} \mathrm{yr}^{-1}\right]=\beta+\alpha \times \log L_{\text {line }}\left[L_{\odot}\right]$ depending on galaxy type, i.e., metal-poor dwarf galaxies, HII/starburst galaxies, composite, or AGN sources, ULIRGs, and high-redshift galaxies.

\begin{tabular}{|c|c|c|c|c|}
\hline SFR calibrator & Number of galaxies & Slope & Intercept & $1 \sigma$ dispersion $[\mathrm{dex}]$ \\
\hline \multicolumn{5}{|c|}{ SFR calibration: entire literature sample } \\
\hline [CII] & 530 & $1.01 \pm 0.02$ & $-6.99 \pm 0.14$ & $0.42(2.6)$ \\
\hline$[\mathrm{OI}]_{63}$ & 150 & $1.00 \pm 0.03$ & $-6.79 \pm 0.22$ & $0.42(2.6)$ \\
\hline$[\mathrm{OIIII}]_{88}{ }^{a}$ & 83 & $1.12 \pm 0.06$ & $-7.48 \pm 0.42$ & $0.66(4.6)$ \\
\hline \multicolumn{5}{|c|}{ SFR calibration: metal-poor dwarf galaxies } \\
\hline [CII] & 42 & $0.80 \pm 0.05$ & $-5.73 \pm 0.32$ & $0.37(2.3)$ \\
\hline$[\mathrm{OI}]_{63}$ & 31 & $0.91 \pm 0.05$ & $-6.23 \pm 0.30$ & $0.27(1.9)$ \\
\hline$[\mathrm{OIIII}]_{88}{ }^{a}$ & 28 & $0.92 \pm 0.05$ & $-6.71 \pm 0.33$ & $0.30(2.0)$ \\
\hline \multicolumn{5}{|c|}{ SFR calibration: HII/starburst galaxies } \\
\hline [CII] & 184 & $1.00 \pm 0.04$ & $-7.06 \pm 0.33$ & $0.27(1.9)$ \\
\hline$[\mathrm{OI}]_{63}$ & 41 & $0.89 \pm 0.06$ & $-6.05 \pm 0.44$ & $0.20(1.6)$ \\
\hline$[\mathrm{OIII}]_{88}{ }^{a}$ & 9 & $0.69 \pm 0.09$ & $-3.89 \pm 0.63$ & $0.23(1.7)$ \\
\hline \multicolumn{5}{|c|}{ SFR calibration: composite/AGN sources } \\
\hline [CII] & 212 & $0.90 \pm 0.04$ & $-6.09 \pm 0.29$ & $0.37(2.3)$ \\
\hline$[\mathrm{OI}]_{63}$ & 37 & $0.76 \pm 0.09$ & $-5.08 \pm 0.73$ & $0.35(2.2)$ \\
\hline$[\mathrm{OIII}]_{88}{ }^{a}$ & 20 & $0.87 \pm 0.14$ & $-5.46 \pm 0.98$ & $0.35(2.2)$ \\
\hline \multicolumn{5}{|c|}{ SFR calibration: ULIRGs } \\
\hline [CII] & 65 & $1.0^{b}$ & $-6.28 \pm 0.04$ & $0.31(2.0)$ \\
\hline$[\mathrm{OI}]_{63}$ & 35 & $1.0^{b}$ & $-6.23 \pm 0.06$ & $0.33(2.1)$ \\
\hline$[\mathrm{OIII}]_{88}$ & 23 & $1.0^{b}$ & $-5.80 \pm 0.09$ & $0.40(2.5)$ \\
\hline \multicolumn{5}{|c|}{ SFR calibration: high-redshift $(z>0.5)$} \\
\hline [CII $]$ & 27 & $1.18 \pm 0.19$ & $-8.52 \pm 1.92$ & $0.40(2.5)$ \\
\hline$[\mathrm{OI}]_{63}$ & 6 & $1.0^{b}$ & $-7.03 \pm 0.29$ & $0.64(4.4)$ \\
\hline$[\mathrm{OIII}]_{88}$ & 3 & $1.0^{b}$ & $-6.89 \pm 0.30$ & $0.42(2.6)$ \\
\hline
\end{tabular}

Notes. The first and second columns indicate the FIR fine-structure line(s) and number of galaxies used in the SFR calibration, with the slope(s) $\alpha$, intercept $\beta$, and dispersion of the best-fit line for the different samples presented in Cols. 3, 4, and 5. Between parentheses, we note the uncertainty factor on the derived SFR estimates corresponding to the dispersion in the relation. ${ }^{(a)}$ We only rely on Herschel observations for the SFR calibrations for [OIII $]_{88}$ because of the significant difference found between the calibration of the Herschel and ISO instruments (see Sect. A.4). ${ }^{(b)}$ The number of galaxies and/or FIR line luminosity range was insufficient to constrain the slope and intercept of the best-fit line. Therefore, the fitting procedure was performed for a fixed slope of 1.

\subsection{SFR-L line calibrations}

For the sample of literature data, we derive SFR calibrations for each of the fine-structure lines $[\mathrm{CII}]$, $[\mathrm{OI}]_{63}$, and $[\mathrm{OIII}]_{88}$, as well as combinations of all lines based on the IDL procedure MPFITFUN and similar functions as defined in Eqs. (3) and (4). To identify a correlation between the SFR and FIR line luminosities, we again require that the parameter $\alpha$ is determined at the $>5 \sigma$ significance level. The best-fit SFR calibrations are presented in Table 3 for each of the different galaxy populations along with the number of galaxies used for the calibration, the slope, and intercept of the best-fit line and the dispersion (or the uncertainty on the SFR estimate in parentheses). The SFR calibrations for the entire galaxy sample allow us to compare the different FIR lines and their applicability to trace the star formation across a large sample of galaxy populations. Although all correlations are significant, the large dispersion in the SFR$L_{\text {line }}$ relations (ranging from 0.42 to $0.66 \mathrm{dex}$ ) immediately tells us that $[\mathrm{CII}],[\mathrm{OI}]_{63}$, and $[\mathrm{OIII}]_{88}$ are fairly unreliable SFR tracers when calibrated for the entire literature sample. In particular, the link of the $[\mathrm{OIII}]_{88}$ emission with the SFR appears to depend strongly on galaxy type.

To improve the applicability of each of the FIR lines as an SFR diagnostic, we derive separate SFR calibrations for each of the different galaxy populations in the literature sample, i.e., dwarf galaxies, HII/starburst galaxies, composite/AGN sources, ULIRGs and high-redshift galaxies. All galaxy subpopulations are exclusive, i.e., the ULIRG population consists of starburst, composite and AGN galaxies, but the starburst and AGN samples do not contain ULIRGs to prevent SFR calibrations biased by the line deficits observed in ULIRGs. For every galaxy population, we attempted to fit a combination of FIR emission lines to probe the SFR (according to formula 4) with the aim of decreasing the scatter in the SFR calibrations. The majority of line combinations did not result in an improvement of the scatter, suggesting that other FIR cooling lines are necessary to supplement the $[\mathrm{CII}],[\mathrm{OI}]_{63}$, and $[\mathrm{OIII}]_{88}$ emission and, thus, trace a more complete cooling budget. While the three finestructure lines $\left([\mathrm{CII}],[\mathrm{OI}]_{63},[\mathrm{OIII}]_{88}\right)$ under investigation in this paper are the brightest FIR lines in our sample of metal-poor dwarfs, other composite line tracers might be more appropriate for high-energy sources like AGNs, starbursts, and ULIRGs (e.g., $[\mathrm{NII}]_{122,205},[\mathrm{NIII}]_{57}$, higher-J CO lines,...). Farrah et al. (2013) find that $[\mathrm{OI}]_{63,145}$ and $[\mathrm{NII}]_{122}$ are the most reliable SFR tracers for a sample of ULIRGs, while Zhao et al. (2013) have shown that $[\mathrm{NII}]_{205}$ is a potentially powerful SFR indicator in local luminous infrared galaxies (LIRGs) as well as in the more distant Universe.

\subsection{Prescriptions for different galaxy populations}

Compared to the scatter in the SFR calibrations for the entire literature sample, the dispersion for each of the separate galaxy populations is significantly reduced (see Table 3 ). Given that the dispersion in the SFR relations also differs significantly among galaxy populations and for the different FIR lines, the correlation 
between the SFR and FIR lines is clearly dependent on galaxy type. As a guideline, we briefly summarize the reliability of the three FIR fine-structure lines $[\mathrm{CII}],[\mathrm{OI}]_{63}$, and $[\mathrm{OIII}]_{88}$ to trace the SFR in each of the following galaxy populations. In case knowledge of the source classification is lacking, the calibrations derived for the $[\mathrm{CII}]$ and $[\mathrm{OI}]_{63}$ lines for the entire source sample (see top part of Table 3) will provide the most reliable SFR estimates with an uncertainty of factor 2.6.

\subsubsection{Metal-poor dwarf galaxies}

The most reliable estimate of the SFR in metal-poor dwarf galaxies can be derived from the $[\mathrm{OI}]_{63}$ luminosity following the calibration:

$\log S F R=-6.23+0.91 \times \log L_{[\mathrm{OI}]}$,

with an uncertainty factor of $\sim 1.9$. The star formation activity can be traced with an uncertainty of factor $\sim 2$ and $\sim 2.3$ from the $[\mathrm{OIII}]_{88}$ and $[\mathrm{CII}]$ lines, respectively, based on:

$\log S F R=-6.71+0.92 \times \log L_{[\mathrm{OIII}]}$,

and

$\log S F R=-5.73+0.80 \times \log L_{[\mathrm{CII}]}$.

All SFR calibrations for metal-poor dwarf galaxies have shallower slopes compared to the entire literature sample, due to their decreasing FIR line luminosity toward lower metal abundances (see Sect. 4.3).

\subsubsection{Starburst galaxies}

The $[\mathrm{CII}]$ and $[\mathrm{OI}]_{63}$ lines can estimate the SFR in starburst galaxies within uncertainty factors of 1.9 and 1.6 following the calibrations:

$\log S F R=-7.06+1.00 \times \log L_{[\mathrm{CII}]}$

and

$\log S F R=-6.05+0.89 \times \log L_{[\mathrm{OI}]}$.

The SFR calibration for [CII] is not very different from previous calibrations obtained by De Looze et al. (2011) and Sargsyan et al. (2012) for normal-star-forming galaxies and starbursts (see Fig. 10), which suggests that the [CII] line is linked to star formation in all galaxies extending from low levels of star formation activity $\left(S F R \sim 0.1 M_{\odot} \mathrm{yr}^{-1}\right)$ to extremely active starbursts $\left(S F R \sim 100 M_{\odot} \mathrm{yr}^{-1}\right)$.

For $[\mathrm{OIII}]_{88}$, we only have nine $[\mathrm{OIII}]_{88}$ line fluxes from Herschel after excluding the ISO measurements, resulting in the following SFR calibration with an uncertainty factor of $\sim 1.7$ on the estimated SFR:

$\log S F R=-3.89+0.69 \times \log L_{[\mathrm{OIII}]}$.

Since $[\mathrm{OIII}]_{88}$ emission requires highly ionized gas of low density, it is not surprising that the $[\mathrm{OIII}]_{88}$ emission is weaker in starburst galaxies (with an average $[\mathrm{OIII}]_{88} /[\mathrm{OI}]_{63}$ line ratio of 0.4 in starburst as compared to 3 in dwarfs), where gas densities are also higher and mean free path lengths are shorter. Although the hard radiation to ionize $\mathrm{O}^{+}$is likely present in local starbursts, the radiation is produced in compact, dusty regions, prohibiting the high-energy photons to reach the lower density gas surrounding dense cores (e.g., Abel et al. 2009).

\subsubsection{Composite/AGN sources}

The SFR calibrations are more dispersed for composite and AGN sources compared to starburst galaxies. The substantial scatter might be due to a possible contribution from dust heated by the AGN to the total infrared luminosity (e.g., Sargsyan et al. 2012). Alternatively, some AGNs appear to show line deficits similar to ULIRGs caused by highly charged dust grains that limit the photoelectric heating efficiency (Tielens \& Hollenbach 1985a; Malhotra et al. 1997; Negishi et al. 2001; Croxall et al. 2012; Farrah et al. 2013) and/or high dust-to-gas opacities due to an increased average ionization parameter (e.g., Graciá-Carpio et al. 2011; Díaz-Santos et al. 2013; Farrah et al. 2013). Part of the dispersion for the $[\mathrm{OI}]_{63}$ line might be caused by selfabsorption and optical depth effects as well as excitation through shocks ${ }^{11}$.

The star formation activity in AGNs can be constrained up to a factor of $\sim 2.3$ based on all three lines:

$\log S F R=-6.09+0.90 \times \log L_{[\mathrm{CII}]}$,

$\log S F R=-5.08+0.76 \times \log L_{[\mathrm{OI}]}$ and

$\log S F R=-5.46+0.87 \times \log L_{[\mathrm{OIII}]}$.

Several combinations of FIR lines, in particular for $[\mathrm{OIII}]_{88}$, result in SFR calibrations with reduced scatter. With the $[\mathrm{OIII}]_{88}$ line being on average $\sim 5$ times fainter than [CII], we believe the results are an artifact of the fitting procedure and do not have any physical interpretation.

\subsubsection{Ultra-luminous infrared galaxies (ULIRGs)}

Since the ULIRG sample does not cover a sufficient range in luminosity to constrain the slope of the SFR calibration, we fix the slope to a value of 1 (similar to the slope for the entire literature sample) and determine the intercept from the fitting procedure. The SFR calibrations for ULIRGs are offset by about 0.5 to 1.0 dex from starbursts and AGNs due to line deficits relative to their total infrared luminosity, which are caused either by the compactness of the size of starburst regions (e.g., Graciá-Carpio et al. 2011; Díaz-Santos et al. 2013; Farrah et al. 2013) and/or enhanced grain charging in regions with high $G_{0} / n_{\mathrm{H}}$ values (Tielens \& Hollenbach 1985a; Malhotra et al. 1997; Negishi et al. 2001; Croxall et al. 2012; Farrah et al. 2013). The occurrence of line deficits has been shown to coincide with the transition between two different modes of star formation (Graciá-Carpio et al. 2011), i.e., the star-forming disk galaxies populating the main sequence in the gas-star formation diagrams and ultra-luminous gas-rich mergers with elevated levels of star formation for the same gas fractions (Daddi et al. 2010; Genzel et al. 2010).

With a fixed slope of 1 , the SFR can be determined from the $[\mathrm{CII}],[\mathrm{OI}]_{63}$, and $[\mathrm{OIII}]_{88}$ luminosities within uncertainty factors of $2,2.1$, and 2.5 , respectively, using the calibrations:

$$
\begin{aligned}
& \log S F R=-6.28+1.0 \times \log L_{[\mathrm{CII}]}, \\
& \log S F R=-6.23+1.0 \times \log L_{[\mathrm{OI}]} \text { and } \\
& \log S F R=-5.80+1.0 \times \log L_{[\mathrm{OIII}]} .
\end{aligned}
$$

The SFR calibrations derived from our sample of ULIRGs are offset from the SFR calibrations reported by Farrah et al. (2013)

11 The literature data for AGNs and ULIRGs mostly correspond to total galaxy values, whereas the link between the SFR and $[\mathrm{OI}]_{63}$ line might be more dispersed zooming in into the central regions of galaxies hosting AGNs. 

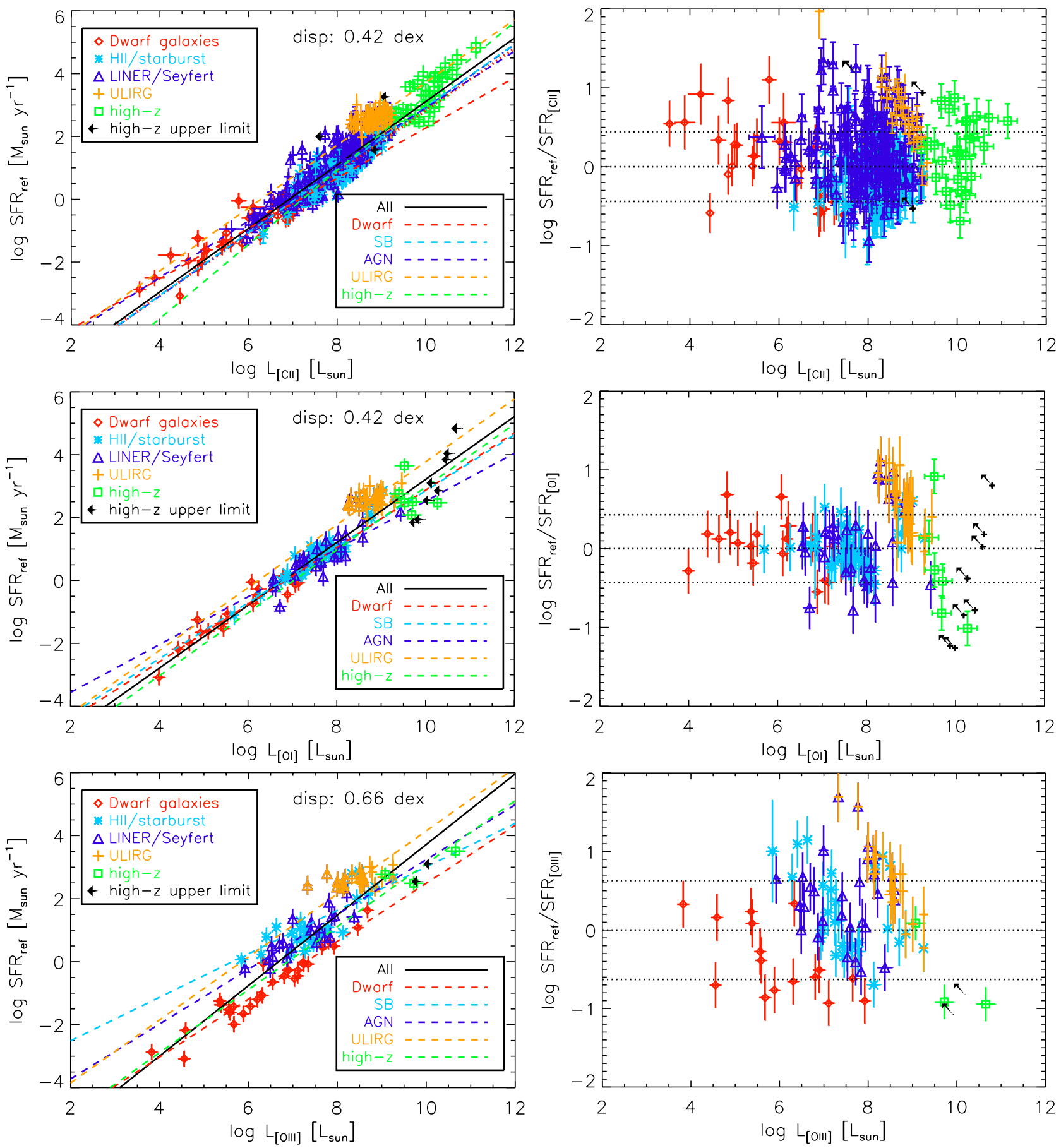

Fig. 10. Left: SFR calibrations based on a literature sample of different galaxy populations for FIR fine-structure lines ([CII] (top), [OI $]_{63}$ (middle), $[\mathrm{OIII}]_{88}$ (bottom). DGS dwarf galaxies, HII/starburst galaxies and composite, LINER or AGN sources are presented as red diamonds, blue asterisks, and purple triangles, respectively. ULIRGs with $L_{\mathrm{FIR}}>10^{12} L_{\odot}$ are indicated as orange crosses. High-redshift sources can be identified as green squares while upper limits for high-redshift objects are shown as black arrows. The solid black line shows the best-fit relation for the entire galaxy sample, while the dashed red, cyan, purple, orange, and green curves represent the SFR calibrations derived for separate galaxy populations, i.e., dwarf galaxies, starbursts, AGNs, ULIRGs, and high-redshift sources, respectively. The $1 \sigma$ dispersion of the entire galaxy sample around the best fit is indicated in the bottom right corner of each panel. Right: dispersion plots indicating the logarithmic distance between the SFR estimates obtained from the reference SFR tracer and the FIR line emission. The $1 \sigma$ dispersion of the entire literature sample is indicated as dashed black lines. The top panel also includes previous SFR calibrations reported in De Looze et al. (2011) (dotted red line) and Sargsyan et al. (2012) (dash-dotted blue line).

in the sense that our SFR estimates are 2 to 4 times higher. Given that our literature sample contains the same ULIRGs presented in Farrah et al. (2013), we believe the difference in the SFR estimate can be attributed to the reference SFR tracer that was used to calibrate the SFR relations. While we rely on the TIR luminosity and the SFR(TIR) calibration presented in Hao et al. (2011), Farrah et al. (2013) use the PAH luminosity and the SFR(PAH) relation presented in Farrah et al. (2007). 


\subsubsection{High-redshift galaxies}

As [CII] observations in high-redshift galaxies have been more popular than other FIR fine-structure lines, we can report a relatively reliable SFR calibration for the [CII] line, based on:

$\log S F R=-8.52+1.18 \times \log L_{[\mathrm{CII}]}$.

Most high-redshift sources follow the trend of local starbursts and AGNs but with significant dispersion (0.40 dex), which results in an uncertainty factor on the SFR estimate of about 2.5. The large scatter can be attributed to some high-redshift galaxies, revealing similar [CII] deficits as ULIRGs. Relying on the warmer temperatures inferred for high-redshift sources (e.g., Magdis et al. 2012), it might not be surprising that [CII] is incapable of tracing the SFR accurately because of the presence of strong radiation fields.

For the $[\mathrm{OI}]_{63}$ and $[\mathrm{OIII}]_{88}$ lines, the literature high-redshift sample did not contain a sufficient number of objects to constrain the slope and intercept in our fitting procedure. Therefore, the slope was fixed to a value of 1 , which is similar to the slope in the SFR calibrations for the entire literature sample. The SFR calibrations for $[\mathrm{OI}]_{63}$ and $[\mathrm{OIII}]_{88}$ determined in this way are:

$\log S F R=-7.03+1.0 \times \log L_{[\mathrm{OI}]}$

and

$\log S F R=-6.89+1.0 \times \log L_{[\mathrm{OIII}]}$.

The scatter in the SFR relations for $[\mathrm{OI}]_{63}$ quickly increases with only six high-redshift detections resulting in an uncertainty factor of $\sim 4.4$ on the SFR estimate. The $[\mathrm{OI}]_{63}$ detections from Sturm et al. (2010), Coppin et al. (2012), and Ferkinhoff et al. (2014) suggest that the line emission is significantly brighter at high-redshift compared to the SFR calibration derived in the local Universe, which could imply that the ISM in those early Universe objects is warmer and denser compared to average conditions in the local Universe. The $[\mathrm{OI}]_{63}$ line might easily become optically thick, however, and could be hampered by other excitation mechanisms (e.g., shocks) in dusty highredshift galaxies, especially during merger episodes. We furthermore need to caution that the few $[\mathrm{OI}]_{63}$ detections of highredshift galaxies might be biased toward hot, dense objects given the difficulty to detect $[\mathrm{OI}]_{63}$ at high redshift. One exception is the intermediate redshift $(z=0.59)$ galaxy IRAS F16413+3954 (Dale et al. 2004), which shows a similar [OI] $]_{63}$ deficit as local ULIRGs. More $[\mathrm{OI}]_{63}$ detections at high-redshift sources are mandatory to infer the behavior of this line with the star formation activity in early Universe objects.

Based on the high-redshift [OIII $]_{88}$ detections and upper limits reported in the literature for five galaxies (Ivison et al. 2010b; Ferkinhoff et al. 2010; Valtchanov et al. 2011), the [OIII $]_{88}$ line might be a potentially powerful tracer of the star formation activity in the early Universe in the absence of [CII] and with a similar degree of uncertainty on the SFR estimate (factor of $~ 2.6$ ). Since hard radiation is required to ionize $\mathrm{O}^{+}$, it is not surprising that the $[\mathrm{OIII}]_{88}$ line is bright in high-redshift sources, which are known to harbor strong radiation fields (e.g., Magdis et al. 2012). Aside from the compact star-forming regions, high-redshift sources could have low-density components where the chemistry and heating is regulated by the hard radiation field.

\section{Conclusions}

Based on Herschel observations of low-metallicity dwarf galaxies from the Dwarf Galaxy Survey, we have analyzed the applicability of FIR fine-structure lines to reliably trace the star formation activity. More specifically, we investigated whether three of the brightest cooling lines in the DGS sample ([CII], $\left.[\mathrm{OI}]_{63},[\mathrm{OIII}]_{88}\right)$ are linked to the star formation rate as probed through a composite SFR tracer (GALEX FUV+MIPS $24 \mu \mathrm{m}$ ). We briefly summarize the results of our analysis:

- On spatially resolved galaxy scales, the $[\mathrm{OIII}]_{88}$ line shows the tightest correlation with the SFR (0.25 dex), which provides determination of the SFR with an uncertainty factor of 1.6. Also, $[\mathrm{OI}]_{63}$ is a reasonably good SFR tracer with an uncertainty factor of 1.7 on the SFR estimate. The spatially resolved relation between [CII] and the SFR is heavily dispersed and does not allow us to constrain the SFR within a factor of 2 .

- The dispersion in the SFR calibrations results from the diversity in ISM conditions (i.e., density and ionization state of the gas) for the DGS sample covering a wide range in metallicity, rather than from variations within one single galaxy on spatially resolved scales.

- On global galaxy scales, the dispersion in the $S F R-L_{[\mathrm{CII}]}$ relation $(0.38 \mathrm{dex})$ is again worse compared to the $[\mathrm{OI}]_{63}$ $(0.25 \mathrm{dex})$ and $[\mathrm{OIII}]_{88}(0.30 \mathrm{dex})$ lines. The $[\mathrm{OI}]_{63}$ line is the most reliable overall SFR indicator in galaxies of subsolar metallicity with an uncertainty factor of 1.8 on the SFR estimate, while the SFR derived from $[\mathrm{OIII}]_{88}$ is uncertain by a factor of 2. The [CII] line is not considered a reliable SFR tracer in galaxies of low metal abundance.

- The scatter in the $S F R-L_{[\mathrm{CII}]}$ relation increases toward low metallicities, warm dust temperatures, and large filling factors of diffuse, highly ionized gas. Due to the porosity of the ISM and the exposure to hard radiation fields, an increased number of ionizing photons is capable of ionizing gas at large distances from the star-forming regions, which favors line cooling through ionized gas tracers such as $[\mathrm{OIII}]_{88}$. The photoelectric efficiency might, furthermore, reduce in lowmetallicity environments due to grain charging and/or increased photon escape fractions.

- On spatially resolved scales, we can reduce the scatter in the SFR calibration by combining the emission from multiple FIR lines. Ideally, we want to probe the emission from all cooling lines that constitute the total gas cooling budget.

Based on the assembly of literature data, we furthermore analyze the applicability of fine-structure lines [CII], [OI $]_{63}$, and $[\mathrm{OIII}]_{88}$ to probe the SFR (as traced by the TIR luminosity) in HII/starburst galaxies, AGNs, ULIRGs, and high-redshift objects:

- The $[\mathrm{CII}]$ and $[\mathrm{OI}]_{63}$ lines are considered to be the most reliable SFR tracers to recover the star formation activity in starburst galaxies with uncertainty factors of 1.9 and 1.6. The [OIII] line, on the other hand, is weak and the SFR calibration could not be well constrained because of the low number of Herschel $[\mathrm{OIII}]_{88}$ detections in starbursts.

- All three FIR lines can recover the SFR from composite or AGN sources within an uncertainty of factor $\sim 2.3$. The increased scatter in the SFR calibrations for AGNs (as compared to starbursts) might result from a possible AGN contribution to the total infrared luminosity (used to derive the SFR). Alternatively, some AGNs might show line deficits similar to ULIRGs.

- ULIRGs are offset from the SFR calibrations for starbursts and AGNs due to line deficits relative to their total infrared luminosity and, therefore, require separate SFR calibrations. 
The star formation rate in ULIRGs is preferentially traced through [CII] and [OI $]_{63}$ line emission, providing SFR estimates with uncertainties of factor $\sim 2$, while the SFR([OIII]) estimate is uncertain by a factor of 2.5 .

- At high-redshift, we can only reliably determine an SFR calibration for the $[\mathrm{CII}]$ line (with an uncertainty factor of $\sim 2.5$ on the SFR estimate) because of the low number of observations for the other lines. The relatively few detections of $[\mathrm{OI}]_{63}$ and $[\mathrm{OIII}]_{88}$ appear to be bright at high-redshift, suggesting that the $[\mathrm{OI}]_{63}$ and $[\mathrm{OIII}]_{88}$ lines are also potentially powerful tracers of the SFR at high redshift, but more detections are mandatory to acquire conclusive evidence.

Acknowledgements. I.D.L. is a postdoctoral researcher of the FWO-Vlaanderen (Belgium). V.L. is supported by a CEA/Marie Curie Eurotalents fellowship. This research was supported by the Agence Nationale pour la Recherche (ANR) through the programme SYMPATICO (Programme Blanc Projet ANR-11-BS560023). PACS has been developed by a consortium of institutes led by MPE (Germany) and including UVIE (Austria); KU Leuven, CSL, IMEC (Belgium); CEA, LAM (France); MPIA (Germany); INAFIFSI/ OAA/OAP/OAT, LENS, SISSA (Italy); IAC (Spain). This development has been supported by the funding agencies BMVIT (Austria), ESA-PRODEX (Belgium), CEA/CNES (France), DLR (Germany), ASI/INAF (Italy), and CICYT/ MCYT (Spain). SPIRE has been developed by a consortium of institutes led by Cardiff University (UK) and including Univ. Lethbridge (Canada); NAOC (China); CEA, LAM (France); IFSI, Univ. Padua (Italy); IAC (Spain); Stockholm Observatory (Sweden); Imperial College London, RAL, UCL-MSSL, UKATC, Univ. Sussex (UK); and Caltech, JPL, NHSC, Univ. Colorado (USA). This development has been supported by national funding agencies: CSA (Canada); NAOC (China); CEA, CNES, CNRS (France); ASI (Italy); MCINN (Spain); SNSB (Sweden); STFC and UKSA (UK); and NASA (USA).

\section{References}

Abel, N. P., Dudley, C., Fischer, J., Satyapal, S., \& van Hoof, P. A. M. 2009, ApJ, 701,1147

Aggarwal, K. M., \& Keenan, F. P. 1999, ApJS, 123, 311

Alonso-Herrero, A., Rieke, G. H., Rieke, M. J., et al. 2006, ApJ, 650, 835

Aniano, G., Draine, B. T., Gordon, K. D., \& Sandstrom, K. 2011, PASP, 123, 1218

Annibali, F., Tosi, M., Monelli, M., et al. 2009, AJ, 138, 169 Asplund, M., Grevesse, N., Sauval, A. J., \& Scott, P. 2009, AR\&A, 47, 481 Baugh, C. M., Lacey, C. G., Frenk, C. S., et al. 2005, MNRAS, 356, 1191 Bendo, G. J., Draine, B. T., Engelbracht, C. W., et al. 2008, MNRAS, 389, 629 Bendo, G. J., Wilson, C. D., Pohlen, M., et al. 2010, A\&A, 518, L65 Bendo, G. J., Boselli, A., Dariush, A., et al. 2012a, MNRAS, 419, 1833 Bendo, G. J., Galliano, F., \& Madden, S. C. 2012b, MNRAS, 423, 197 Boquien, M., Calzetti, D., Combes, F., et al. 2011, AJ, 142, 111 Boselli, A., Gavazzi, G., Lequeux, J., \& Pierini, D. 2002, A\&A, 385, 454 Boselli, A., Lequeux, J., \& Gavazzi, G. 2004, A\&A, 428, 409 Boselli, A., Boissier, S., Cortese, L., \& Gavazzi, G. 2008, ApJ, 674, 742 Brauher, J. R., Dale, D. A., \& Helou, G. 2008, ApJS, 178, 280 Calzetti, D., Armus, L., Bohlin, R. C., et al. 2000, ApJ, 533, 682 Calzetti, D., Kennicutt, R. C., Jr., Bianchi, L., et al. 2005, ApJ, 633, 871 Calzetti, D., Kennicutt, R. C., Engelbracht, C. W., et al. 2007, ApJ, 666, 870 Calzetti, D., Wu, S.-Y., Hong, S., et al. 2010, ApJ, 714, 1256

Cannon, J. M., \& Skillman, E. D. 2004, ApJ, 610, 772

Condon, J. J., Cotton, W. D., Greisen, E. W., et al. 1998, AJ, 115, 1693

Cannon, J. M., Walter, F., Armus, L., et al. 2006, ApJ, 652, 1170

Coppin, K. E. K., Danielson, A. L. R., Geach, J. E., et al. 2012, MNRAS, 427, 520

Cormier, D., 2012, Ph.D. thesis, Université Paris Diderot (Paris 7), France

Cormier, D., Madden, S. C., Hony, S., et al. 2010, A\&A, 518, L57 Cortese, L., Boissier, S., Boselli, A., et al. 2012, A\&A, 544, A101

Cox, P., Krips, M., Neri, R., et al. 2011, ApJ, 740, 63

Crawford, M. K., Genzel, R., Townes, C. H., \& Watson, D. M. 1985, ApJ, 291, 755

Croxall, K. V., Smith, J. D., Wolfire, M. G., et al. 2012, ApJ, 747, 81

Daddi, E., Elbaz, D., Walter, F., et al. 2010, ApJ, 714, L118

Davé, R. 2008, MNRAS, 385, 147

Dale, D. A., \& Helou, G. 2002, ApJ, 576, 159

Dale, D. A., Helou, G., Brauher, J. R., et al. 2004, ApJ, 604, 565

De Breuck, C., Maiolino, R., Caselli, P., et al. 2011, A\&A, 530, L8

de Jong, T., Klein, U., Wielebinski, R., \& Wunderlich, E. 1985, A\&A, 147, L6
De Looze, I., Baes, M., Bendo, G. J., Cortese, L., \& Fritz, J. 2011, MNRAS, 416, 2712

Díaz-Santos, T., Armus, L., Charmandaris, V., et al. 2013, ApJ, 774, 68

Draine, B. T., Dale, D. A., Bendo, G., et al. 2007, ApJ, 663, 866

Engelbracht, C. W., Gordon, K. D., Rieke, G. H., et al. 2005, ApJ, 628, L29

Engelbracht, C. W., Blaylock, M., Su, K. Y. L., et al. 2007, PASP, 119, 994 Engelbracht, C. W., Rieke, G. H., Gordon, K. D., et al. 2008, ApJ, 678, 804 Farrah, D., Bernard-Salas, J., Spoon, H. W. W., et al. 2007, ApJ, 667, 149

Farrah, D., Lebouteiller, V., Spoon, H. W. W., et al. 2013, ApJ, 776, 38 Ferkinhoff, C., Hailey-Dunsheath, S., Nikola, T., et al. 2010, ApJ, 714, L147 Ferkinhoff, C., Brisbin, D., Parshley, S., et al. 2014, ApJ, 780, 142

Fioc, M., \& Rocca-Volmerange, B. 1999, A\&A, 344, 393

Fischer, J., Sturm, E., González-Alfonso, E., et al. 2010, A\&A, 518, L41 Fitzpatrick, E. L. 1999, PASP, 111, 63

Franx, M., Illingworth, G. D., Kelson, D. D., van Dokkum, P. G., \& Tran, K.-V. 1997, ApJ, 486, L75

Galametz, M., Madden, S., Galliano, F., et al. 2009, A\&A, 508, 645

Galametz, M., Madden, S. C., Galliano, F., et al. 2010, A\&A, 518, L55

Galametz, M., Madden, S. C., Galliano, F., et al. 2011, A\&A, 532, A56

Galliano, F., Madden, S. C., Jones, A. P., et al. 2003, A\&A, 407, 159

Galliano, F., Madden, S. C., Jones, A. P., Wilson, C. D., \& Bernard, J.-P. 2005, A\&A, 434, 867

Galliano, F., Dwek, E., \& Chanial, P. 2008, ApJ, 672, 214

Gavazzi, G., Boselli, A., Scodeggio, M., Pierini, D., \& Belsole, E. 1999, MNRAS, 304, 595

Gavazzi, G., Boselli, A., Donati, A., Franzetti, P., \& Scodeggio, M. 2003, A\&A, 400, 451

Genzel, R., Tacconi, L. J., Gracia-Carpio, J., et al. 2010, MNRAS, 407, 2091

Gil de Paz, A., Madore, B. F., \& Pevunova, O. 2003, ApJS, 147, 29

Glover, S. C. O., \& Clark, P. C. 2014, MNRAS, 437, 9

Goldsmith, P. F., Langer, W. D., Pineda, J. L., \& Velusamy, T. 2012, ApJS, 203, 13

Gordon, K. D., Engelbracht, C. W., Rieke, G. H., et al. 2008, ApJ, 682, 336

Graciá-Carpio, J., Sturm, E., Hailey-Dunsheath, S., et al. 2011, ApJ, 728, L7

Griffin, M. J., Abergel, A., Abreu, A., et al. 2010, A\&A, 518, L3

Groves, B., Krause, O., Sandstrom, K., et al. 2012, MNRAS, 426, 892

Hailey-Dunsheath, S., Nikola, T., Stacey, G. J., et al. 2010, ApJ, 714, L162

Hao, C.-N., Kennicutt, R. C., Johnson, B. D., et al. 2011, ApJ, 741, 124

Heiles, C. 1994, ApJ, 436, 720

Helou, G. 2000, Infrared Space Astronomy, Today and Tomorrow, eds. F. Casali, J. Lequeux, \& F. David, Les Houches Summer School, 70, 337

Helou, G., Soifer, B. T., \& Rowan-Robinson, M. 1985, ApJ, 298, L7

Helou, G., Roussel, H., Appleton, P., et al. 2004, ApJS, 154, 253

Herrera-Camus, R., Fisher, D. B., Bolatto, A. D., et al. 2012, ApJ, 752, 112

Hodge, P., Smith, T., Eskridge, P., MacGillivray, H., \& Beard, S. 1991, ApJ, 379, 621

Hollenbach, D., \& McKee, C. F. 1989, ApJ, 342, 306

Houck, J. R., Charmandaris, V., Brandl, B. R., et al. 2004, ApJS, 154, 211

Hunt, L. K., Dyer, K. K., \& Thuan, T. X. 2005, A\&A, 436, 837

Hunter, D. A., Kaufman, M., Hollenbach, D. J., et al. 2001, ApJ, 553, 121

Hwang, H. S., Geller, M. J., Diaferio, A., \& Rines, K. J. 2012, ApJ, 752, 64

Iono, D., Yun, M. S., Elvis, M., et al. 2006, ApJ, 645, L97

Israel, F. P., \& Maloney, P. R. 2011, A\&A, 531, A19

Israel, F. P., Maloney, P. R., Geis, N., et al. 1996, ApJ, 465, 738

Ivison, R. J., Smail, I., Papadopoulos, P. P., et al. 2010a, MNRAS, 404, 198

Ivison, R. J., Swinbank, A. M., Swinyard, B., et al. 2010b, A\&A, 518, L35 Jackson, D. C., Cannon, J. M., Skillman, E. D., et al. 2006, ApJ, 646, 192

Jones, A. P., Tielens, A. G. G. M., \& Hollenbach, D. J. 1996, ApJ, 469, 740

Kaufman, M. J., Wolfire, M. G., Hollenbach, D. J., \& Luhman, M. L. 1999, ApJ, 527,795

Kaufman, M. J., Wolfire, M. G., \& Hollenbach, D. J. 2006, ApJ, 644, 283

Kennicutt, R. C., Jr. 1998, ARA\&A, 36, 189

Kennicutt, R. C., \& Evans, N. J. 2012, ARA\&A, 50, 531

Kennicutt, R. C., Jr., Armus, L., Bendo, G., et al. 2003, PASP, 115, 928

Kennicutt, R. C., Jr., Lee, J. C., Funes, S. J., et al. 2008, ApJS, 178, 247

Kennicutt, R. C., Jr., Hao, C.-N., Calzetti, D., et al. 2009, ApJ, 703, 1672

Kraemer, K., Jackson, J. M., \& Lane, A. P. 1996, BAAS, 28, 1302

Kroupa, P., \& Weidner, C. 2003, ApJ, 598, 1076

Lacey, C. G., Baugh, C. M., Frenk, C. S., et al. 2008, MNRAS, 385, 1155

Larson, R. B. 1998, MNRAS, 301, 569

Lebouteiller, V., Kunth, D., Lequeux, J., et al. 2004, A\&A, 415, 55

Lebouteiller, V., Cormier, D., Madden, S. C., et al. 2012, A\&A, 548, A91

Lee, J. C., Hwang, H. S., \& Ko, J. 2013, ApJ, 774, 62

Lequeux, J. 1989, Evolution of Galaxies: Astronomical Observations (Berlin,

New York: Springer Verlag), 333, 147

Leroy, A. K., Walter, F., Brinks, E., et al. 2008, AJ, 136, 2782

Li, Y., Crocker, A. F., Calzetti, D., et al. 2013, ApJ, 768, 180

Lisenfeld, U., Israel, F. P., Stil, J. M., \& Sievers, A. 2002, A\&A, 382, 860 
Ilse De Looze et al.: The applicability of far-infrared fine-structure lines as star formation rate tracers

Luhman, M. L., Satyapal, S., Fischer, J., et al. 1998, ApJ, 504, L11

Luhman, M. L., Satyapal, S., Fischer, J., et al. 2003, ApJ, 594, 758

Madden, S. C. 2000, New Astron. Rev., 44, 249

Madden, S. C., Poglitsch, A., Geis, N., Stacey, G. J., \& Townes, C. H. 1997, ApJ, 483, 200

Madden, S. C., Galliano, F., Jones, A. P., \& Sauvage, M. 2006, A\&A, 446, 877

Madden, S. C., Rémy-Ruyer, A., Galametz, M., et al. 2013, PASP, 125, 600

Magdis, G. E., Daddi, E., Béthermin, M., et al. 2012, ApJ, 760, 6

Maiolino, R., Cox, P., Caselli, P., et al. 2005, A\&A, 440, L51

Maiolino, R., Caselli, P., Nagao, T., et al. 2009, A\&A, 500, L1

Malhotra, S., Helou, G., Stacey, G., et al. 1997, ApJ, 491, L27

Malhotra, S., Kaufman, M. J., Hollenbach, D., et al. 2001, ApJ, 561, 766

Markwardt, C. B. 2009, Astronomical Data Analysis Software and Systems XVIII, ASP Conf. Ser., 411, 251

Marsden, G., Borys, C., Chapman, S. C., Halpern, M., \& Scott, D. 2005, MNRAS, 359, 43

Mateo, M. L. 1998, ARA\&A, 36, 435

McKee, C. F., \& Ostriker, E. C. 2007, ARA\&A, 45, 565

Moshir, M., et al. 1990, IRAS Faint Source Catalogue, version 2.0 (1990), 0

Moustakas, J., \& Kennicutt, R. C., Jr. 2006, ApJS, 164, 81

Murphy, E. J., Condon, J. J., Schinnerer, E., et al. 2011, ApJ, 737, 67

Negishi, T., Onaka, T., Chan, K.-W., \& Roellig, T. L. 2001, A\&A, 375, 566

Östlin, G., Hayes, M., Kunth, D., et al. 2009, AJ, 138, 923

Ouchi, M., Ellis, R., Ono, Y., et al. 2013, ApJ, 778, 102

Parkin, T. J., Wilson, C. D., Schirm, M. R. P., et al. 2013, ApJ, 776, 65

Péquignot, D. 2008, A\&A, 478, 371

Pérez-González, P. G., Kennicutt, R. C., Jr., Gordon, K. D., et al. 2006, ApJ, 648, 987

Pflamm-Altenburg, J., Weidner, C., \& Kroupa, P. 2007, ApJ, 671, 1550

Pilbratt, G. L., Riedinger, J. R., Passvogel, T., et al. 2010, A\&A, 518, L1

Pilyugin, L. S., \& Thuan, T. X. 2005, ApJ, 631, 231

Planck Collaboration XVI. 2014, A\&A, in press DOI: 10.1051/0004-6361/201321591

Poglitsch, A., Krabbe, A., Madden, S. C., et al. 1995, ApJ, 454, 293

Poglitsch, A., Herrmann, F., Genzel, R., et al. 1996, ApJ, 462, L43

Poglitsch, A., Waelkens, C., Geis, N., et al. 2010, A\&A, 518, L2

Rawle, T. D., Egami, E., Bussmann, R. S., et al. 2014, ApJ, 783, 59

Rémy-Ruyer, A., Madden, S. C., Galliano, F., et al. 2013, A\&A, 557, A95

Rémy-Ruyer, A., Madden, S. C., Galliano, F., et al. 2014, A\&A, 563, A31

Riechers, D. A., Walter, F., Carilli, C. L., \& Lewis, G. F. 2009, ApJ, 690, 463

Riechers, D. A., Bradford, C. M., Clements, D. L., et al. 2013, Nature, 496, 329
Rieke, G. H., Alonso-Herrero, A., Weiner, B. J., et al. 2009, ApJ, 692, 556

Röllig, M., Ossenkopf, V., Jeyakumar, S., Stutzki, J., \& Sternberg, A. 2006, A\&A, 451, 917

Roy, J.-R., \& Kunth, D. 1995, A\&A, 294, 432

Sanders, D. B., \& Mirabel, I. F. 1996, ARA\&A, 34, 749

Sanders, D. B., Mazzarella, J. M., Kim, D.-C., Surace, J. A., \& Soifer, B. T. 2003, AJ, 126, 1607

Salim, S., Rich, R. M., Charlot, S., et al. 2007, ApJS, 173, 267

Sandstrom, K. M., Bolatto, A. D., Bot, C., et al. 2012, ApJ, 744, 20

Sargsyan, L., Lebouteiller, V., Weedman, D., et al. 2012, ApJ, 755, 171

Schlafly, E. F., \& Finkbeiner, D. P. 2011, ApJ, 737, 103

Schlegel, D. J., Finkbeiner, D. P., \& Davis, M. 1998, ApJ, 500, 525

Schurer, A., Calura, F., Silva, L., et al. 2009, MNRAS, 394, 2001

Silk, J., \& Werner, M. W. 1969, ApJ, 158, 185

Smith, M. W. L., Eales, S. A., Gomez, H. L., et al. 2012, ApJ, 756, 40

Stacey, G. J., Geis, N., Genzel, R., et al. 1991, ApJ, 373, 423

Stacey, G. J., Hailey-Dunsheath, S., Ferkinhoff, C., et al. 2010, ApJ, 724, 957

Stierwalt, S., Armus, L., Surace, J. A., et al. 2013, ApJS, 206, 1

Sturm, E., Verma, A., Graciá-Carpio, J., et al. 2010, A\&A, 518, L36

Swinbank, A. M., Karim, A., Smail, I., et al. 2012, MNRAS, 427, 1066

Thuan, T. X., Sauvage, M., \& Madden, S. 1999, ApJ, 516, 783

Thuan, T. X., Hibbard, J. E., \& Lévrier, F. 2004, AJ, 128, 617

Tielens, A. G. G. M., \& Hollenbach, D. 1985a, ApJ, 291, 722

Tielens, A. G. G. M., \& Hollenbach, D. 1985b, ApJ, 291, 747

Tosi, M., Aloisi, A., Mack, J., \& Maio, M. 2007, Proc. IAU Symp., 235, 65

Tully, R. B. 1988, Nearby galaxies catalog (Cambridge, New York: Cambridge University Press)

Valtchanov, I., Virdee, J., Ivison, R. J., et al. 2011, MNRAS, 415, 3473

Verma, A., Charmandaris, V., Klaas, U., Lutz, D., \& Haas, M. 2005, Space Sci. Rev., 119, 355

Wagg, J., Carilli, C. L., Wilner, D. J., et al. 2010, A\&A, 519, L1

Wagg, J., Wiklind, T., Carilli, C. L., et al. 2012, ApJ, 752, L30

Wilkins, S. M., Hopkins, A. M., Trentham, N., \& Tojeiro, R. 2008, MNRAS, 391,363

Wolfire, M. G., Hollenbach, D., McKee, C. F., Tielens, A. G. G. M., \& Bakes, E. L. O. 1995, ApJ, 443, 152

Wright, E. L. 2006, PASP, 118, 1711

Wu, H., Cao, C., Hao, C.-N., et al. 2005, ApJ, 632, L79

Zhao, Y., Lu, N., Xu, C. K., et al. 2013, ApJ, 765, L13

Zhu, Y.-N., Wu, H., Cao, C., \& Li, H.-N. 2008, ApJ, 686, 155 
Table A.1. Overview of the different reference SFR calibrations.

\begin{tabular}{|c|c|c|c|}
\hline \multicolumn{4}{|c|}{ SFR calibrations } \\
\hline$L_{\mathrm{x}}\left[\mathrm{erg} \mathrm{s}^{-1}\right]$ & Age range $[\mathrm{Myr}]$ & $C_{\mathrm{x}}$ & Ref. \\
\hline FUV $_{\text {corr }}$ & $0-10-100$ & 43.35 & 1,2 \\
\hline $\mathrm{H} \alpha_{\text {corr }}$ & $0-3-10$ & 41.27 & 1,2 \\
\hline $24 \mu \mathrm{m}$ & $0-5-100$ & 42.69 & 3 \\
\hline $70 \mu \mathrm{m}$ & $0-5-100$ & 43.23 & 4 \\
\hline TIR & $0-5-100$ & 43.41 & 1,2 \\
\hline \multicolumn{4}{|c|}{ Composite SFR calibrations } \\
\hline${\text { Composite } \text { tracer }^{a}}^{a}$ & $v$ & $1 \sigma \operatorname{disp}[\operatorname{dex}]$ & Ref. \\
\hline$L_{\mathrm{FUV}, \mathrm{obs}}+v L_{24}^{b, c}$ & 3.89 & 0.13 & 1 \\
\hline$L_{\mathrm{FUV}, \mathrm{obs}}+v L_{1.4 \mathrm{GHz}}$ & $7.2 \times 10^{14}$ & 0.14 & 1 \\
\hline$L_{\mathrm{FUV}, \mathrm{obs}}+v L_{\mathrm{TIR}}$ & 0.27 & 0.09 & 1 \\
\hline$L_{\mathrm{H} \alpha, \mathrm{obs}}+v L_{24} b$ & 0.020 & 0.12 & 5 \\
\hline
\end{tabular}

Notes. In the first part, the SFR calibration coefficients are provided for several SFR indicators following the prescription $\log S F R=\log L_{\mathrm{x}}-$ $\log C_{\mathrm{x}}$. The column "Age range" specifies the lower age limit, the mean age, and the age of stars below which $90 \%$ of the emission is contributed. The last column refers to the literature work(s) reporting the calibrations. The second part gives the calibration factors for several composite SFR tracers used to correct the unobscured SFR indicators for extinction, with Cols. 2 and 3 presenting the calibration coefficients $v$ and average dispersion in the calibrations. ${ }^{(a)}$ The luminosities of composite tracers are in units of erg s${ }^{-1}$, except $L_{1.4 \mathrm{GHz}}$ which is in units of erg s${ }^{-1} \mathrm{~Hz}^{-1}$. ${ }^{(b)}$ The SFR calibration is valid for the emission from MIPS $24 \mu \mathrm{m}$ or IRAS $25 \mu \mathrm{m}$ filters, due to the excellent correspondence that exists between both bands (Kennicutt et al. 2009). ${ }^{(c)}$ Two calibrations based on FUV and MIPS $24 \mu \mathrm{m}$ have been reported in the literature. Zhu et al. (2008) found a calibration coefficient $v=6.31$ based on a sample of 187 star-forming non-AGN galaxies, while Hao et al. (2011) found a lower scaling factor $v=3.89$, calibrated on a sample of 144 galaxies retained from the spectrophotometric survey by Moustakas \& Kennicutt (2006) (97 objects) and SINGS (47 objects, Kennicutt et al. 2003). No clear reason has been found to explain the difference in calibration coefficients derived by Zhu et al. (2008) and Hao et al. (2011).

References. (1) Hao et al. (2011); (2) Murphy et al. (2011); (3) Rieke et al. (2009); (4) Calzetti et al. (2010) ; (5) Kennicutt et al. (2009).

\section{Appendix A: Comparison between different SFR tracers for the DGS sample}

\section{A.1. Limitations of SFR tracers in metal-poor dwarf galaxies}

In this work, we rely on the most recent calibrations reported in Kennicutt \& Evans (2012), which are calibrated for an initial mass function (IMF) characterized by a broken power law with a slope of -2.35 from 1 to $100 M_{\odot}$ and -1.3 between 0.1 and $1 M_{\odot}$ (Kroupa \& Weidner 2003). Table A.1 gives an overview of the reference SFR calibrations used in this work. The SFR calibrations based on single tracers in the first part of Table A.1 predict the SFR in $M_{\odot} \mathrm{yr}^{-1}$ following the prescription $\log S F R=\log L_{\mathrm{x}}-\log C_{\mathrm{x}}$, where $L_{\mathrm{x}}$ is the SFR indicator in units of erg s${ }^{-1}$ and $C_{\mathrm{x}}$ represents the calibration coefficients for a specific SFR diagnostic. The second part of Table A.1 provides the calibrations to correct unobscured SFR indicators for extinction.

The empirically derived SFR calibrations from Table A.1 assume a constant star formation rate over timescales comparable to or longer than the lifetime of stars to which the SFR tracers are sensitive. The age range for the SFR tracers used in this analysis are summarized in the second column of Table A.1 and were adopted from Kennicutt \& Evans (2012), with the first, second, and third number representing the lower age boundary, the mean age, and the age of stars below which $90 \%$ of the emission is contributed, respectively. For observations covering an entire galaxy, we sample a wide range in age across the different starforming complexes, sufficient to maintain a constant level of star formation activity when averaging out over different galaxy regions. With low-mass galaxies being dominated by one or only few HII regions and furthermore characterized by bursty star formation histories (e.g., Mateo 1998; Fioc \& Rocca-Volmerange 1999), the assumption of constant star formation rate might not be valid because of the insufficient sampling of different ages. The SFR calibrations might, therefore, no longer hold. In particular, on spatially resolved scales (i.e., few hundreds of parsec or the size of a typical HII region for nearby DGS sources achieved in Herschel observations), the sampling of different ages will not suffice to sample the entire age range to maintain a constant star formation activity (i.e., constant SFR across the entire age range) and the SFR calibrations might break down. Therefore, we caution the interpretation of the SFR in the analysis of metal-poor dwarf galaxies, in particular for the spatially resolved analysis in Sect. 3, in the sense that the unobscured SFR obtained from GALEX FUV might underestimate the "true" level of star formation activity throughout the galaxy.

Other than the constant SFR, empirical SFR calibrations assume the universality of the initial mass function (IMF). Pflamm-Altenburg et al. (2007) question this universality of the IMF on global galaxy scales, especially in dwarf galaxies, arguing that the maximum stellar mass in a star cluster is limited by the total mass of the cluster with the latter being constrained by the SFR. Neglecting this effect could result in an underestimation of the SFR by up to 3 orders of magnitude. Since the data currently at hand do not allow a proper investigation of any possible deviations from universal IMF, we apply and derive SFR calibrations in this paper under the assumption of universality of the IMF, but keep in mind that possible systematic effects might bias our analysis. The extendibility of SFR calibrations to the early Universe might furthermore be affected by deviations from this universal IMF, with indications for a top heavy IMF at high-redshift (Larson 1998; Baugh et al. 2005; Davé 2008; Lacey et al. 2008; Wilkins et al. 2008).

\section{A.2. Comparison of unobscured SFR tracers: GALEX FUV and $H \alpha$}

The FUV and $\mathrm{H} \alpha$ fluxes are commonly-used tracers of the unobscured star formation. The applicability of FUV as a star formation rate tracer might, however, be affected by the recent star formation history in dwarfs, which is often bursty and dominated by few, giant HII regions (e.g., Mateo 1998; Fioc \& RoccaVolmerange 1999). With $\mathrm{H} \alpha$ being sensitive to the emission of OB stars with mean ages $\sim 10^{7} \mathrm{yr}$ and FUV tracing the emission of massive A stars with ages $\sim 10^{8} \mathrm{yr}$ (Lequeux 1989), H $\alpha$ might be more appropriate as SFR tracer in dwarf galaxies with a particular bursty star formation history.

In Fig. A.1, we compare the ratio of the SFR as obtained from the SFR calibrators FUV + MIPS $24 \mu \mathrm{m}$ and $\mathrm{H} \alpha+$ MIPS $24 \mu \mathrm{m}$ (see SFR relations in Table A.1) as a function of oxygen abundance. Total $\mathrm{H} \alpha$ fluxes are taken from Gil de Paz et al. (2003); Moustakas \& Kennicutt (2006); Kennicutt et al. (2008); Östlin et al. (2009). We determine the best fit to the data points based on linear regression fits using the IDL procedure MPFITEXY, which is based on the nonlinear least-squares fitting package MPFIT (Markwardt 2009). The best-fit line and a perfect one-to-one correlation are indicated as dotted red, and 
Ilse De Looze et al.: The applicability of far-infrared fine-structure lines as star formation rate tracers

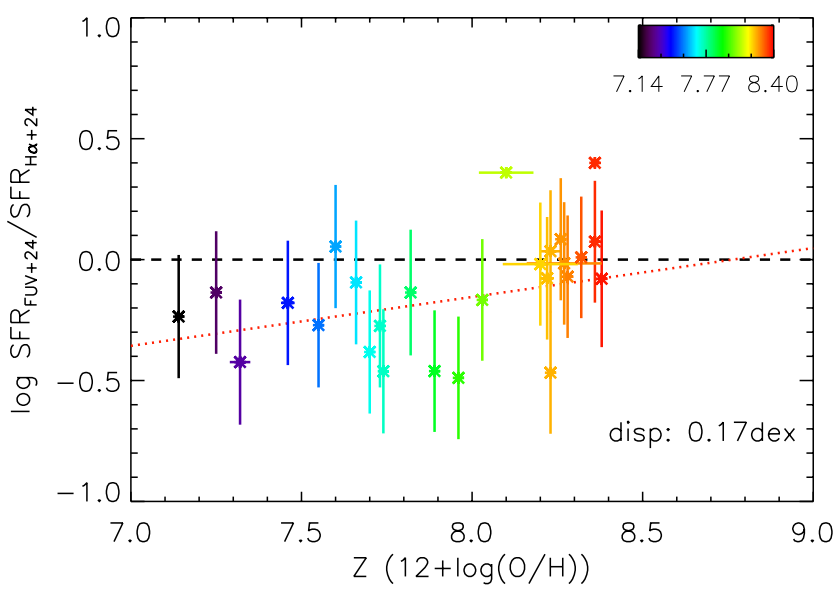

Fig. A.1. Comparison between the ratio of the SFR as obtained from the SFR calibrators FUV + MIPS $24 \mu \mathrm{m}$ and $\mathrm{H} \alpha+$ MIPS $24 \mu \mathrm{m}$ as a function of oxygen abundance. Galaxies are color-coded according to metallicity with increasing oxygen abundances ranging from black then blue, green and yellow to red colors. The dotted red, and dashed black, lines represent the best fit and a perfect one-to-one correlation, respectively. The dispersion around the best fit is indicated in the bottom right corner.

dashed black lines. The dispersion around the best fit is indicated in the bottom right corner.

For galaxies with oxygen abundances $12+\log (\mathrm{O} / \mathrm{H}) \geq 8.1$, the two composite SFR tracers seem to provide consistent estimates of the SFR, while below $12+\log (\mathrm{O} / \mathrm{H})<8.1$ galaxies start to deviate from a perfect one-to-one correlation. Due to the lower stellar masses of metal-poor dwarfs, their star formation history is dominated by only few giant HII regions and, thus, heavily dependent on the time delay since the last burst of star formation. With FUV tracing the unobscured star formation over a timescale of 10 to 100 Myr (Kennicutt 1998; Calzetti et al. 2005; Salim et al. 2007), the SFR derived from FUV emission will be lower compared to $\mathrm{H} \alpha$ in galaxies characterized by a single recent burst of star formation $(<10 \mathrm{Myr})$ over a timescale of 100 Myr. Therefore, $\mathrm{H} \alpha$ is considered a better SFR calibrator in low-mass dwarfs, which are dominated by single HII regions. The correspondence between SFR estimates from FUV and $H \alpha$ emission for galaxies with $12+\log (\mathrm{O} / \mathrm{H}) \geq 8.1 \mathrm{might}$ indicate an age effect, where the latter objects are rather in a post-starburst phase and the most recent starburst occurred more than $10 \mathrm{Myr}$ ago. The unavailability of $\mathrm{H} \alpha$ maps (only global $\mathrm{H} \alpha$ fluxes could be retrieved from the literature for most galaxies) prevents us from using $\mathrm{H} \alpha$ as reference SFR tracer, since we are unable to recover the $\mathrm{H} \alpha$ emission that corresponds to the areas in galaxies covered by our Herschel observations. Therefore, FUV is used as reference SFR tracer for this analysis, bearing in mind that the conversion to SFR might break down for low-mass metal-poor dwarfs where FUV will on average underestimate the SFR derived from $\mathrm{H} \alpha$ by $50 \%$ (see Fig. A.1).

\section{A.3. Comparison of obscured SFR tracers: IRAC8 $\mu \mathrm{m}$, MIPS $24 \mu \mathrm{m}, L_{T I R}$, and $1.4 \mathrm{GHz}$}

Different monochromatic and multiband data in the infrared and radio wavelength domain can be used to trace the obscured star formation component. Here, we compare IRAC $8 \mu \mathrm{m}$, MIPS $24 \mu \mathrm{m}$, PACS $70 \mu \mathrm{m}$, the total infrared luminosity, and radio continuum emission at $1.4 \mathrm{GHz}$. Total IRAC $8 \mu \mathrm{m}$ flux densities have been adopted from Rémy-Ruyer et al. (in prep.). The DGS sample has been observed by the Herschel PACS (70, 100,
$160 \mu \mathrm{m})$ and SPIRE $(250,350,500 \mu \mathrm{m})$ photometers in all continuum bands. Details about the observing strategy, the applied data reduction techniques and aperture photometry results are presented in Rémy-Ruyer et al. (2013). Total infrared luminosities $L_{\mathrm{TIR}}$ are taken from Madden et al. (2013), as determined from Spitzer bands using the prescriptions in Dale \& Helou (2002). Radio continuum measurements are retrieved from the NRAO VLA Sky Survey (NVSS) catalog (Condon et al. 1998), Cannon \& Skillman (2004), Thuan et al. (2004), and Hunt et al. (2005).

The emission from PAHs usually dominates the IRAC $8 \mu \mathrm{m}$ band in metal-rich galaxies. In low-metallicity galaxies, the IRAC $8 \mu \mathrm{m}$ band might also contain an important contribution from the warm continuum emission from very small grains. Since the PAH emission has been observed to be under-luminous below $12+\log (\mathrm{O} / \mathrm{H}) \sim 8.1$ (Boselli et al. 2004; Engelbracht et al. 2005; Jackson et el. 2006; Madden et al. 2006; Draine et al. 2007; Engelbracht et al. 2008; Galliano et al. 2008), in combination with the uncertainty to quantifying the $8 \mu \mathrm{m}$ band in terms of PAH and VSG contribution, the IRAC $8 \mu \mathrm{m}$ band is considered an unreliable SFR calibrator for the DGS sample covering a wide range in metallicity. Calzetti et al. (2007) could indeed identify that the sensitivity of the IRAC $8 \mu \mathrm{m}$ band to metallicity is about one order of magnitude worse compared to MIPS $24 \mu \mathrm{m}$. The weak PAH emission toward lower metallicities is not directly related to the lower metal abundance but rather emanates from the generally strong and hard radiation fields in low-metallicity systems destroying and/or ionizing PAHs (e.g., Gordon et al. 2008; Sandstrom et al. 2012). Other than its dependence on metallicity (or thus radiation field), PAH emission tends to be inhibited in regions of strong star formation activity while it can be several times more luminous compared to other star formation rate tracers in regions with relatively weak or nonexistent star formation (e.g., Calzetti et al. 2005; Bendo et al. 2008; Gordon et al. 2008). Prior to any conversion of IRAC $8 \mu \mathrm{m}$ emission to SFR, the band emission needs to be corrected for any stellar contribution. Given that the contribution from the stellar continuum could be substantial in low-abundance galaxies, this will make the correction using standard recipes (e.g., Helou et al. 2004) highly uncertain. All together, we argue that the IRAC $8 \mu \mathrm{m}$ is not appropriate as SFR indicator in our sample of dwarf galaxies with widely varying metallicities.

The most common monochromatic tracer of obscured star formation is MIPS $24 \mu \mathrm{m}$ emission, which generally originates from a combination of stochastically heated very small grains (VSGs) and large grains at an equilibrium temperature of $\sim 100 \mathrm{~K}$. For a grain size distribution similar to our Galaxy, we expect large equilibrium grains only to start dominating the MIPS $24 \mu \mathrm{m}$ emission above a threshold of $G_{0} \sim 100$, where $G_{0}$ is the scaling factor of the interstellar radiation field, expressed relative to the FUV interstellar radiation field from 6 to $13.6 \mathrm{eV}$ for the solar neighborhood in units of Habing flux, i.e., $1.6 \times 10^{-3} \mathrm{erg} \mathrm{s}^{-1} \mathrm{~m}^{-2}$. The emission in the MIPS $24 \mu \mathrm{m}$ band has been shown to be well-correlated with other star formation rate tracers on both local scales (Calzetti et al. 2007; Leroy et al. 2008) and global scales (e.g., Calzetti et al. 2005, 2007; Wu et al. 2005; Alonso-Herrero et al. 2006; Pérez-González et al. 2006; Zhu et al. 2008; Kennicutt et al. 2009; Rieke et al. 2009; Hao et al. 2011) and directly traces the ongoing star formation over a timescale of $\sim 10$ Myr (Calzetti et al. 2005; Pérez-González et al. 2006; Calzetti et al. 2007).

Since the grain properties and size distribution has been shown to be sensitive to the metallicity of galaxies (Lisenfeld et al. 2002; Galliano et al. 2003, 2005), we need to verify 
whether MIPS $24 \mu \mathrm{m}$ is an appropriate SFR tracer for the DGS sample. In low-metallicity objects, small grain sizes $(\$ 3 \mathrm{~nm})$ start to dominate the $24 \mu \mathrm{m}$ emission compared to larger dust grains (Lisenfeld et al. 2002; Galliano et al. 2003, 2005), due to the fragmentation of these larger dust grains through shocks experienced in the turbulent ISM. The hard radiation field in low-metallicity galaxies (see Sect. 4.3) furthermore increases the maximum temperature of stochastically heated grains and shifts the peak of the SED to shorter wavelengths, boosting the MIPS $24 \mu \mathrm{m}$ flux (e.g., Thuan et al. 1999; Houck et al. 2004; Galametz et al. 2009, 2011). To verify the influence of metallicity on the MIPS $24 \mu \mathrm{m}$ band emission, we compare the estimated star formation rates obtained from MIPS $24 \mu \mathrm{m}$ to other SFR indicators, which should not be biased (or at least less) by variations in the dust composition across galaxies with different metal abundances.

Toward longer wavelengths $(70,100,160 \mu \mathrm{m})$, the band emission is dominated by larger dust grains and should not depend strongly on the abundance of very small grains. For larger dust grains a significant fraction of the dust heating might, however, be attributed to more evolved stellar populations, making the link between FIR continuum emission and star formation more dispersed (Bendo et al. 2010; Calzetti et al. 2010; Boquien et al. 2011; Bendo et al. 2012a; Groves et al. 2012; Smith et al. 2012). In a similar way, the total infrared luminosity might be subject to heating from old stars and, therefore, only linked to star formation on much longer timescales. Some individual studies argue that most of the dust heating is provided by star-forming regions in dwarfs (e.g., Galametz et al. 2010; Bendo et al. 2012a), even at wavelengths longward of $160 \mu \mathrm{m}$, suggesting that the longer wavelength data $(70,100,160 \mu \mathrm{m})$ could potentially be reliable star formation rate tracers. More detailed analyses of the dominant heating sources for dust in dwarfs however might be required to conclude on the applicability of FIR continuum bands to trace the SFR. In Fig. A.2, we compare the SFR as estimated from the single continuum bands MIPS $24 \mu \mathrm{m}$ and PACS $70 \mu \mathrm{m}$ (see top panel). The bestfit line and dispersion $(0.28 \mathrm{dex})$ in this plot is mainly dominated by three galaxies (SBS 0335-052, Tol 1214-277, Haro 11), which have the peak of their SED at very short wavelengths and, therefore, the $24 \mu \mathrm{m}$ band will overestimate the SFR while the $70 \mu \mathrm{m}$ emission will underestimate the SFR. The other galaxies seem to follow the one-to-one correlation better with a dispersion of $0.18 \mathrm{dex}$ around the one-to-one correlation (or difference between the two SFR estimates up to $51 \%$ ).

In the central panel of Fig. A.2, we make a similar comparison between the composite SFR tracers FUV $+24 \mu \mathrm{m}$ and FUV+TIR. The best-fit line (with slope $\alpha=0.15$ ) diverges from the one-to-one correlation with the combination of FUV and MIPS $24 \mu \mathrm{m}$ providing higher SFR than inferred from FUV+TIR. We argue that this discrepancy is mainly caused by the fact that the SFR relations for the total infrared luminosity were calibrated based on galaxy samples of normal starforming galaxies with close to solar abundances. They might, therefore, be less appropriate for metal-poor dwarfs, which are typically characterized by steeply rising mid-infrared (MIR) to far-infrared (FIR) slopes and overall SEDs peaking at wavelengths lower than $\sim 60 \mu \mathrm{m}$ (e.g., Thuan et al. 1999; Houck et al. 2004; Galametz et al. 2009, 2011).

Radio continuum emission at $1.4 \mathrm{GHz}$ is dominated by nonthermal synchrotron emission associated with the acceleration of electrons in a galaxy's magnetic field, and, therefore, independent of the grain composition in a galaxy's ISM. The emission at $1.4 \mathrm{GHz}$ is often used as a tracer of star formation, since the
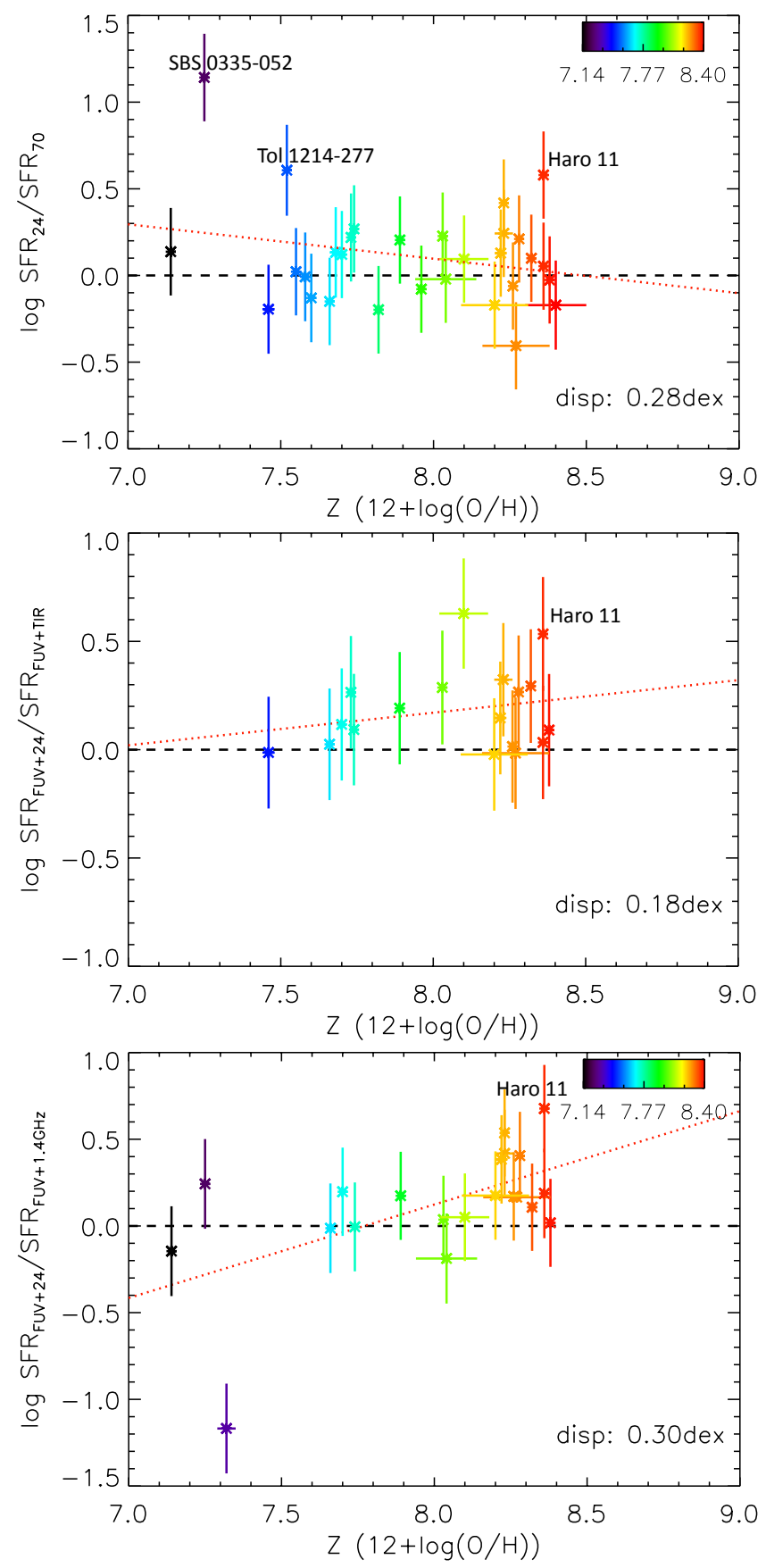

Fig. A.2. Comparison between the ratio of the SFR as obtained from the SFR calibrators MIPS $24 \mu \mathrm{m}$ and PACS $70 \mu \mathrm{m}$ (top), FUV + MIPS $24 \mu \mathrm{m}$ and FUV+TIR (middle) and FUV+MIPS $24 \mu \mathrm{m}$ and FUV $+1.4 \mathrm{GHz}$ (bottom) as a function of oxygen abundance. Galaxies are color-coded according to metallicity with increasing oxygen abundances ranging from black then blue, green and yellow to red colors. The dotted red and dashed black lines represent the best fit and a perfect one-to-one correlation, respectively. The dispersion around the best fit is indicated in the bottom right corner.

optically thin radio synchrotron emission correlates well with the FIR emission (i.e., the FIR-radio correlation; e.g., de Jong et al. 1985; Helou et al. 1985). However, the $1.4 \mathrm{GHz}$ is not really a tracer of obscured star formation since it probes the energy from supernovae associated with star-forming regions and, therefore, rather correlates with the total energy output from star-forming regions, including both obscured and unobscured star formation. Figure A.2 (bottom panel) shows the ratio of SFR estimated from 
$\mathrm{FUV}+24 \mu \mathrm{m}$ and FUV $+1.4 \mathrm{GHz}$ as a function of oxygen abundance (or metallicity), with the black dashed line representing the one-to-one correlation. In general, the SFR obtained from the composite tracer FUV+MIPS $24 \mu \mathrm{m}$ is higher than the SFR estimated from FUV+1.4 GHz. We argue that this deviation can again be attributed to the bursty star formation history in dwarf galaxies. Although the emission at $24 \mu \mathrm{m}$ and $1.4 \mathrm{GHz}$ is sensitive to stars of ages up to at least $100 \mathrm{Myr}$ (see Table A.1), the MIPS $24 \mu \mathrm{m}$ band is dominated by the emission of stars younger than 10 Myr. In view of the recent trigger of star formation that characterizes most dwarf galaxies in our sample, the $1.4 \mathrm{GHz}$ emission will typically underestimate the SFR since it was calibrated for models of constant SFR (or supernova rate) over the past 100 Myr. One exception is galaxy HS $0822+3542$, for which the SFR seems to be underestimated based on FUV+MIPS $24 \mu \mathrm{m}$ data. The strange behavior of this galaxy in the PACS wavebands (Rémy-Ruyer et al. 2013) supports the peculiarity of this object. Another outlier is Haro 11, which shows the highest ratio based on the composite tracer FUV $+24 \mu \mathrm{m}$. We believe that the warmer dust temperatures with a peak in its SED at very short wavelengths ( $40 \mu \mathrm{m}$, Galametz et al. 2009$)$ in this LIRG causes an overestimation of the SFR based on MIPS $24 \mu \mathrm{m}$.

Based on the above comparison between different tracers of obscured star formation, we opted to use the reference SFR tracers FUV and MIPS $24 \mu \mathrm{m}$ for the analysis of the DGS sample. By estimating the SFR from FUV and MIPS $24 \mu \mathrm{m}$ emission, we should be tracing the emission of young stars with ages up to $100 \mathrm{Myr}$. It is possible, however, to get diffuse emission that is not locally heated by star-forming regions in both FUV and MIPS $24 \mu \mathrm{m}$ bands originating from heating by the diffuse interstellar radiation field. This could potentially cause an overestimation of the SFR in diffuse regions and might bias the interpretation of the observed relations between the SFR and FIR line emission, in particular for the spatially resolved analysis of Sect. 3. With the Dwarf Galaxy Survey often not mapping the nearby galaxies completely in the FIR lines but rather focusing on the brightest star-forming regions, we argue that the contribution from diffuse regions and the heating by evolved stellar populations most likely will be limited in many cases. Although, we should keep in mind the possible contribution of diffuse FUV and $24 \mu \mathrm{m}$ emission to the SFR estimates upon analyzing the SFR relations. Similarly, the FUV band might contain residual starlight from evolved stellar populations due to the low level of obscuration in some of the dwarfs. The presence of only few evolved stars in dwarfs (compared to more massive early-type galaxies with large spheroids), however, makes it unlikely that residual starlight of old stars will contribute significantly to the FUV emission.

\section{A.4. Comparison ISO-Herschel}

Since the literature sample is composed of Herschel and ISO fluxes, we need to verify whether the spectroscopic flux calibration of the Herschel PACS and ISO LWS instruments are compatible. Hereto, we assemble the ISO fluxes from the Brauher et al. (2008) catalog for galaxies in our literature sample with Herschel measurements. We only consider galaxies that are classified as unresolved with respect to the LWS beam in Brauher et al. (2008) to minimize the influence of different beam sizes in our comparison. We gather a sample of 44,19 , and 10 sources with [CII], [OI] $]_{63}$ and [OIII $]_{88}$ fluxes from both Herschel and ISO observations. Those galaxies are indicated with a dagger behind their name in Tables B.3, B.4 and B.5. Figure A.3 presents the Herschel-to-ISO line ratios
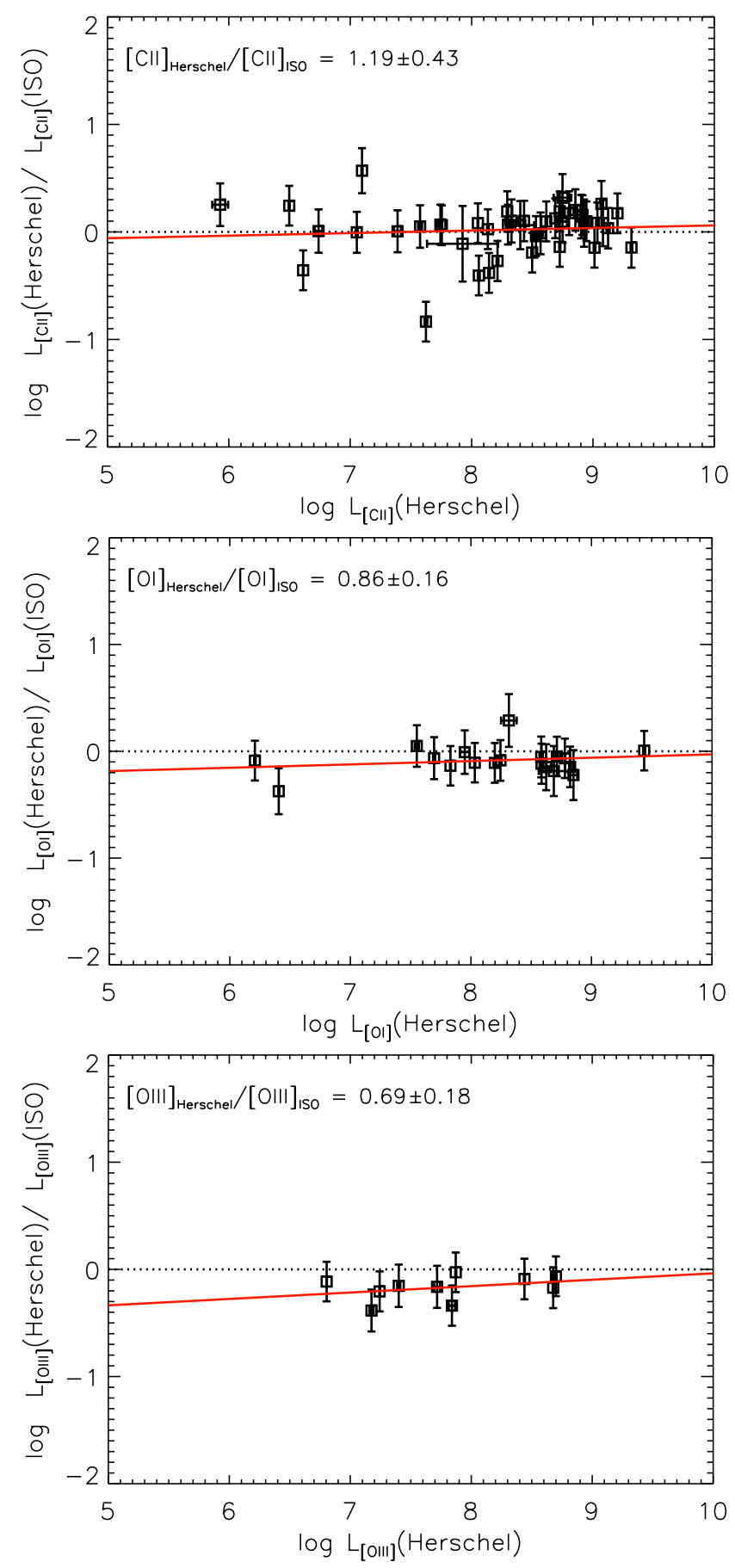

Fig. A.3. Comparison between the Herschel and ISO line fluxes, i.e., the ratio of the Herschel and ISO measurements for [CII] (top), [OI] $]_{63}$ (middle), and [OIII $]_{88}$ (bottom) as a function of Herschel line luminosities. The mean and standard deviation of the Herschel-to-ISO flux ratio is indicated in the top left corner of each panel. The best-fit line and a perfect one-to-one correlation are indicated as solid red and dotted black lines, respectively.

for $[\mathrm{CII}]$ (top), $[\mathrm{OI}]_{63}$ (middle) and $[\mathrm{OIII}]_{88}$ (bottom). Based on the 44 measurements for [CII], we find that the Herschel fluxes are on average $1.19 \pm 0.43$ higher compared to the ISO measurements. The best-fit line (see solid red line in Fig. A.3, top panel) lies close to the one-to-one correlation. Overall, the Herschel and ISO [CII] fluxes are consistent with each other within the error bars, in particular for the higher luminosity sources. Based on the $19[\mathrm{OI}]_{63}$ and ten $[\mathrm{OIII}]_{88}$ measurements, 
we infer that the Herschel measurements are on average lower by a factor of $0.86 \pm 0.16$ and $0.69 \pm 0.18$, respectively, compared to the ISO fluxes. For the [OI $]_{63}$ line, the Herschel PACS and ISO LWS measurements still agree within the error bars, but for the $[\mathrm{OIII}]_{88}$ line the discrepancy between Herschel and ISO measurements is considered significant. Although the larger ISO LWS beam $\left(\sim 80^{\prime \prime}\right)$ could collect more flux compared to the PACS beam for the $[\mathrm{OI}]_{63}$ and $[\mathrm{OIII}]_{88}$ lines $\left(F W H M \sim 9.5^{\prime \prime}\right)$, we would expect to see similar behavior for the $[\mathrm{CII}]$ line
(PACS FWHM 11.5") if the difference in beam size is driving the offset between Herschel and ISO fluxes. The absence of any beam size effects for [CII] makes us conclude that the discrepancy between Herschel and ISO for [OIII] $]_{88}$ can likely be attributed to a difference in calibration. Given the significant difference in $[\mathrm{OIII}]_{88}$ line fluxes, we will only take the $[\mathrm{OIII}]_{88}$ measurements derived from Herschel observations into account for the SFR calibrations.

\section{Appendix B: Tables}

Table B.1. Overview of DGS sources used in the SFR calibrations presented in this paper.

\begin{tabular}{|c|c|c|c|c|c|}
\hline Source & $\begin{array}{c}D^{a} \\
{[\mathrm{Mpc}]}\end{array}$ & $12+\log (\mathrm{O} / \mathrm{H})^{a}$ & $\begin{array}{l}\text { FUV } \\
{[\mathrm{mJy}]}\end{array}$ & $\begin{array}{c}\text { MIPS } 24 \mu \mathrm{m}^{b} \\
{[\mathrm{mJy}]}\end{array}$ & $\begin{array}{c}\text { SFR } \\
{\left[M_{\odot} \mathrm{yr}^{-1}\right]}\end{array}$ \\
\hline IZw $18^{*}$ & 18.2 & 7.14 & $1.482 \pm 0.220$ & $6.1 \pm 0.3^{*}$ & $0.057 \pm 0.021$ \\
\hline SBS 0335-052* & 56.0 & 7.25 & $0.805 \pm 0.119$ & $76.8 \pm 3.2 *$ & $0.890 \pm 0.313$ \\
\hline HS $0822+3542$ & 11.0 & 7.32 & $0.166 \pm 0.025$ & $3.2 \pm 0.3^{*}$ & $0.0031 \pm 0.0011$ \\
\hline UGC 4483 & 3.2 & 7.46 & $\begin{array}{c}1.011 \pm 0.150 \\
{[1.762 \pm 0.261]}\end{array}$ & $10.1 \pm 0.5^{*}$ & $0.0014 \pm 0.0005$ \\
\hline Tol 1214-277* & 120.5 & 7.52 & $0.188 \pm 0.028$ & $6.8 \pm 0.3^{*}$ & $0.543 \pm 0.194$ \\
\hline SBS $1415+437 *$ & 13.6 & 7.55 & $1.124 \pm 0.167$ & $16.8 \pm 1.7$ & $0.030 \pm 0.011$ \\
\hline SBS $1211+540$ & 19.3 & 7.58 & $0.198 \pm 0.029$ & $3.3 \pm 0.3^{*}$ & $0.011 \pm 0.004$ \\
\hline HS $1442+4250$ & 14.4 & 7.60 & $\begin{array}{c}0.590 \pm 0.087 \\
{[1.145 \pm 0.170]}\end{array}$ & $6.6 \pm 0.3^{*}$ & $0.016 \pm 0.006$ \\
\hline VII Zw 403* & 4.5 & 7.66 & $2.485 \pm 0.368$ & $24.9 \pm 2.4$ & $0.007 \pm 0.002$ \\
\hline SBS $1249+493$ & 110.8 & 7.68 & $0.088 \pm 0.013$ & $4.3 \pm 0.3^{*}$ & $0.251 \pm 0.089$ \\
\hline NGC 2366* & 3.2 & 7.70 & $8.627 \pm 1.278$ & $483.7 \pm 13.3$ & $0.022 \pm 0.008$ \\
\hline UM 461* & 13.2 & 7.73 & $0.483 \pm 0.072$ & $34.4 \pm 3.2 *$ & $0.024 \pm 0.009$ \\
\hline Mrk 209* & 5.8 & 7.74 & $1.920 \pm 0.284$ & $40.1 \pm 2.3$ & $0.010 \pm 0.004$ \\
\hline UM 133* & 22.7 & 7.82 & $\begin{array}{c}0.560 \pm 0.083 \\
{[0.978 \pm 0.145]}\end{array}$ & $9.4 \pm 0.5^{*}$ & $0.043 \pm 0.016$ \\
\hline NGC 4861* & 7.5 & 7.89 & $7.911 \pm 1.172$ & $262.7 \pm 10.0$ & $0.085 \pm 0.030$ \\
\hline NGC 6822-Hubble V* & 0.5 & 7.96 & $7.619 \pm 1.129$ & $970.6 \pm 18.1$ & $0.0008 \pm 0.0003$ \\
\hline NGC $1569^{*}$ & 3.1 & 8.02 & $179.284 \pm 26.562$ & $6709.9 \pm 154.4$ & $0.348 \pm 0.124$ \\
\hline Mrk 930* & 77.8 & 8.03 & $1.100 \pm 0.163$ & $152.4 \pm 5.6$ & $3.095 \pm 1.085$ \\
\hline HS $0052+2536^{*}$ & 191.0 & 8.04 & $0.262 \pm 0.039$ & $20.7 \pm 1.2^{*}$ & $2.963 \pm 1.045$ \\
\hline Mrk 1089* & 56.6 & 8.10 & $3.509 \pm 0.520$ & $391.4 \pm 7.6$ & $4.436 \pm 1.558$ \\
\hline NGC 4449* & 4.2 & 8.20 & $73.385 \pm 8.379$ & $1967.1 \pm 19.7$ & $0.226 \pm 0.080$ \\
\hline NGC 625* & 3.9 & 8.22 & $6.253 \pm 0.926$ & $717.1 \pm 16.6$ & $0.038 \pm 0.013$ \\
\hline II Zw $40^{*}$ & 12.1 & 8.23 & $20.374 \pm 3.018$ & $1361.3 \pm 54.4$ & $0.831 \pm 0.294$ \\
\hline Haro $2 *$ & 21.7 & 8.23 & $2.888 \pm 0.428$ & $663.3 \pm 12.8$ & $0.954 \pm 0.334$ \\
\hline NGC 4214* & 2.9 & 8.26 & $34.892 \pm 5.169$ & $1495.7 \pm 29.1$ & $0.063 \pm 0.023$ \\
\hline NGC $1705^{*}$ & 5.1 & 8.27 & $12.768 \pm 1.892$ & $43.9 \pm 0.9$ & $0.038 \pm 0.014$ \\
\hline Haro $3^{*}$ & 19.3 & 8.28 & $3.794 \pm 0.562$ & $714.7 \pm 25.3$ & $0.840 \pm 0.294$ \\
\hline UM 448* & 87.8 & 8.32 & $1.890 \pm 0.280$ & $522.5 \pm 18.2$ & $11.994 \pm 4.193$ \\
\hline UM 311* & 23.5 & 8.36 & $1.422 \pm 0.211$ & $145.2 \pm 6.5$ & $0.291 \pm 0.102$ \\
\hline Haro $11 *$ & 92.1 & 8.36 & $2.398 \pm 0.355$ & $1854.4 \pm 96.3$ & $43.010 \pm 15.022$ \\
\hline NGC $1140^{*}$ & 20.0 & 8.38 & $5.923 \pm 0.877$ & $271.0 \pm 9.1$ & $0.530 \pm 0.189$ \\
\hline HS $2352+2733$ & 116.7 & 8.40 & $0.044 \pm 0.007$ & $2.6 \pm 0.3 *$ & $0.155 \pm 0.055$ \\
\hline
\end{tabular}

Notes. Different galaxies are ordered in increasing order of oxygen abundance. The first, second, and third columns specify the name of the source, distance, and oxygen abundance, respectively. Galaxies from the complete galaxy sample (i.e., with FIR line detections for [CII], [OI] 63 , and $\left.[\mathrm{OIII}]_{88}\right)$ are indicated with an asterisk behind their name. GALEX FUV and MIPS $24 \mu \mathrm{m}$ photometry results are presented in Cols. 4 and 5. The last column gives the SFR, as estimated from the GALEX FUV and MIPS $24 \mu \mathrm{m}$ measurements and the calibrations presented in Hao et al. (2011) and Kennicutt et al. (2009). ${ }^{(a)}$ Distances and oxygen abundances are adopted from Madden et al. (2013). ${ }^{(b)}$ MIPS $24 \mu \mathrm{m}$ flux densities with an asterisk correspond to total galaxy fluxes and were retrieved from Bendo et al. (2012b). 
Ilse De Looze et al.: The applicability of far-infrared fine-structure lines as star formation rate tracers

Table B.2. Overview of literature data for high-redshift galaxies used for the SFR calibrations presented in this paper.

\begin{tabular}{|c|c|c|c|c|c|c|c|}
\hline Source & $z$ & Ref & $\begin{array}{c}{[\mathrm{CII}]} \\
{\left[\log L_{\odot}\right]}\end{array}$ & $\begin{array}{c}{[\mathrm{OI}]_{63}} \\
{\left[\log L_{\odot}\right]}\end{array}$ & $\begin{array}{l}{[\mathrm{OIII}]_{88}} \\
{\left[\log L_{\odot}\right]}\end{array}$ & $\begin{array}{l}\log L_{\mathrm{TIR}} \\
{\left[\log L_{\odot}\right]}\end{array}$ & $\begin{array}{c}\log S F R \\
{\left[\log M_{\odot} \mathrm{yr}^{-1}\right]}\end{array}$ \\
\hline IRAS F16413+3954 & 0.59 & 1 & - & $9.52^{a}$ & - & 13.47 & 3.65 \\
\hline IRAS F04207-0127 & 0.91 & 1 & - & $\leq 10.61$ & - & 13.67 & 3.84 \\
\hline LESS J033217.6-275230 & 1.10 & 2 & - & $\leq 9.87$ & - & 11.67 & 1.84 \\
\hline IRAS F10026+4949 & 1.12 & 3 & 10.44 & - & - & 14.02 & 4.20 \\
\hline 3C 368 & 1.13 & 3 & 10.00 & - & - & 13.14 & 3.31 \\
\hline PG $1206+459$ & 1.16 & 3 & 9.94 & - & - & 13.74 & 3.92 \\
\hline SMM J22471-0206 & 1.16 & 3 & 10.28 & - & - & 13.56 & 3.74 \\
\hline $3 \mathrm{C} 065$ & 1.18 & 3 & $\leq 9.22$ & - & - & 13.09 & 3.26 \\
\hline SMM J123634.51+621241.0 & 1.22 & 3 & 10.20 & - & - & 12.77 & 2.94 \\
\hline LESS J0333294.9-273441 & 1.24 & 2 & - & $\leq 9.99$ & - & 11.76 & 1.94 \\
\hline PG $1241+176$ & 1.27 & 3 & 10.60 & - & - & 13.80 & 3.97 \\
\hline LESS J033155.2-275345 & 1.27 & 2 & - & 9.69 & - & 11.91 & 2.08 \\
\hline MIPS J142824.0+352619 ${ }^{b}$ & 1.30 & $3,4,5$ & 9.85 & 9.53 & - & 12.29 & 2.47 \\
\hline LESS J033331.7-275406 & 1.32 & 2 & - & 9.71 & - & 12.33 & 2.50 \\
\hline IRAS F16348+7037 & 1.33 & 1 & - & $\leq 10.64$ & - & 13.86 & 4.04 \\
\hline IRAS F22231-0512 & 1.40 & 1 & - & $\leq 10.82$ & - & 14.66 & 4.83 \\
\hline $3 \mathrm{C} 446$ & 1.40 & 3 & 11.13 & - & - & 14.66 & 4.83 \\
\hline LESS J033150.84-274438 & 1.61 & 2 & - & $\leq 10.43$ & - & 12.68 & 2.86 \\
\hline LESS J033140.1-275631 & 1.62 & 2 & - & 10.27 & - & 12.29 & 2.47 \\
\hline PKS 0215+015 & 1.72 & 3 & 10.70 & - & - & 14.27 & 4.44 \\
\hline H-ATLAS J091043.1-000322 ${ }^{c}$ & 1.79 & 6 & 9.33 & 9.40 & - & 12.33 & 2.75 \\
\hline RX J094144.51+385434.8 & 1.82 & 3 & 10.38 & - & - & 13.69 & 3.86 \\
\hline SDSS J100038.01+020822.4 & 1.83 & 3 & 10.06 & - & - & 13.13 & 3.30 \\
\hline SWIRE J104704.97+592332.3 & 1.95 & 3 & 10.31 & - & - & 13.13 & 3.30 \\
\hline SWIRE J104738.32+591010.0 & 1.96 & 3 & 10.09 & - & - & 12.73 & 2.91 \\
\hline SMM J2135-0102 ${ }^{d}$ & 2.33 & 7 & 9.75 & $\leq 10.18$ & $\leq 9.91$ & 12.37 & 2.55 \\
\hline H-ATLAS ID $130^{e}$ & 2.63 & 8 & - & $\leq 10.31$ & $\leq 10.22$ & 12.97 & 3.14 \\
\hline SMM J02399-0136 ${ }^{f}$ & 2.81 & 9 & - & - & 10.66 & 13.34 & 3.51 \\
\hline H-ATLAS ID81 $1^{g}$ & 3.04 & 8 & 10.11 & - & 9.76 & 12.36 & 2.53 \\
\hline APM $08279+5255^{h}$ & 3.91 & 9 & - & - & 9.07 & 12.59 & 2.77 \\
\hline H-ATLAS ID141 ${ }^{i}$ & 4.24 & 10 & 9.49 & - & - & 12.79 & 2.87 \\
\hline BRI 1335-0417 & 4.41 & 11 & 10.22 & - & - & 13.74 & 3.91 \\
\hline ALESS 65.1 & 4.42 & 12 & 9.52 & - & - & 12.32 & 2.49 \\
\hline BRI 0952-0115 & 4.43 & 13 & 9.66 & - & - & 12.67 & 2.85 \\
\hline ALESS 61.1 & 4.44 & 12 & 9.19 & - & - & 12.34 & 2.51 \\
\hline BR $1202-0725 \mathrm{~N}$ & 4.69 & 14,15 & 10.00 & - & - & 13.15 & 3.33 \\
\hline BR $1202-0725 \mathrm{~S}$ & 4.69 & 14,15 & 9.82 & - & - & 13.48 & 3.66 \\
\hline LESS J033229.4 & 4.76 & 16 & 10.01 & - & - & 12.78 & 2.96 \\
\hline CI $1358+62^{k}$ & 4.93 & 17 & $\leq 9.98$ & - & - & 12.38 & 2.56 \\
\hline HLSJ091828.6+514223 ${ }^{l}$ & 5.24 & 18 & 10.19 & - & - & 13.27 & 3.44 \\
\hline HFLS3 & 6.34 & 19 & 10.19 & - & - & 13.70 & 3.87 \\
\hline SDSS J1148+52511 & 6.42 & 20 & 9.64 & - & - & 13.41 & 3.58 \\
\hline Himiko & 6.60 & 21 & $\leq 7.75$ & - & - & $\leq 10.80$ & $2.00^{m}$ \\
\hline
\end{tabular}

Notes. The first, second, and third columns specify the name of the source, redshift, and reference to the literature work, respectively. The subsequent columns provide the $[\mathrm{CII}],[\mathrm{OI}]_{63},[\mathrm{OIII}]_{88}$, total infrared $(8-1000 \mu \mathrm{m})$ luminosities as well as star formation rate. Upper limits for undetected FIR lines at high-redshift correspond to $3 \sigma$ upper limits. ${ }^{(a)}$ Only a $2.7 \sigma$ detection. ${ }^{(b)}$ Luminosities have not been corrected for a possible lensing factor, but the galaxy could possibly be lensed up to a factor of 8 (Stacey et al. 2010). ${ }^{(c)}$ Luminosities have been corrected assuming a lensing factor of 18, following Ferkinhoff et al. (2014). ${ }^{(d)}$ We apply the luminosities corrected for lensing reported by Ivison et al. (2010b). ${ }^{(e)}$ Luminosities have been corrected assuming a lensing factor of 6, following the best fit for the lens model reported in Valtchanov et al. (2011). ${ }^{(f)}$ Luminosities have been corrected assuming a lensing factor of 2.38, following Ivison et al. (2010a). ${ }^{(g)}$ Luminosities have been corrected assuming a lensing factor of 25 , following the improved lens model presented in Valtchanov et al. (2011). ${ }^{(h)}$ Luminosities have been corrected assuming a lensing factor of 90, following the most extreme estimate of the lensing factor $(\mu \sim 4-90)$ reported in Riechers et al. (2009). ${ }^{(i)}$ Luminosities have been corrected assuming a lensing factor of 20 , which is the median of the range in amplification factors reported in Cox et al. $(2011)^{(j)}$ Luminosities have been corrected assuming a lensing factor of 4.5, following Maiolino et al. (2009). ${ }^{(k)}$ Luminosities have been corrected assuming a lensing factor of 11 , following the most extreme lensing predictions of Franx et al. (1997). ${ }^{(l)}$ Luminosities have been corrected assuming a lensing factor of 8.9, following Rawle et al. (2014). ${ }^{(m)}$ The SFR is not calculated from $L_{\mathrm{TIR}}$ since Himiko is an optically bright galaxy with low metal and dust content. Therefore, we adapt the SFR estimate $\left(\sim 100 \mathrm{M}_{\odot} \mathrm{yr}^{-1}\right)$ obtained from the stellar population synthesis in Ouchi et al. (2013).

References. (1) Dale et al. (2004); (2) Coppin et al. (2012); (3) Stacey et al. (2010); (4) Hailey-Dunsheath et al. (2010); (5) Sturm et al. (2010); (6) Ferkinhoff et al. (2014); (7) Ivison et al. (2010b); (8) Valtchanov et al. (2011); (9) Ferkinhoff et al. (2010); (10) Cox et al. (2011); (11) Wagg et al. (2010); (12) Swinbank et al. (2012); (13) Maiolino et al. (2009); (14) Iono et al. (2006); (15) Wagg et al. (2012); (16) De Breuck et al. (2011); (17) Marsden et al. (2005); (18) Rawle et al. (2014); (19) Riechers et al. (2013); (20) Maiolino et al. (2005); (21) Ouchi et al. (2013). 
Table B.3. Overview of literature data for dwarf galaxies used for the SFR calibrations presented in this paper.

\begin{tabular}{lccccc}
\hline \hline Source & $\begin{array}{c}D_{L}{ }^{a} \\
{[\mathrm{Mpc}]}\end{array}$ & Ref & $\begin{array}{c}\text { FUV } \\
{[\mathrm{mJy}]}\end{array}$ & $\begin{array}{c}\text { MIPS 24 } \mu \mathrm{m} / \mathrm{IRAS} 25 \mu \mathrm{m} \\
{[\mathrm{mJy}]}\end{array}$ & $\begin{array}{c}\log S F R \\
{\left[\log M_{\odot} \mathrm{yr}^{-1}\right]}\end{array}$ \\
\hline NGC 4713 & 19.9 & 1 & $13.308 \pm 1.972$ & $186.5 \pm 50.4$ & -0.13 \\
NGC 4490 & 8.7 & 1 & $10.248 \pm 1.518$ & $1394.8 \pm 55.9$ & -0.45 \\
NGC 1156 & 7.1 & 1 & $23.307 \pm 3.453$ & $447.1 \pm 18.0$ & -0.74 \\
NGC 4299 & 18.7 & 1 & $8.996 \pm 1.333$ & $210.4 \pm 8.5$ & -0.28 \\
NGC 693 & 23.4 & 1 & $0.973 \pm 0.144$ & $550.0 \pm 50.0$ & -0.08 \\
NGC 814 & 24.1 & 1 & $0.321 \pm 0.048$ & $897.2 \pm 62.8$ & 0.14 \\
NGC 4294 & 17 & 1 & $5.249 \pm 0.778$ & $192.5 \pm 57.8$ & -0.52 \\
VCC 1326 & 17 & 1 & $0.290 \pm 0.043$ & $418.4 \pm 54.4$ & -0.49 \\
NGC 4522 & 17 & 1 & $2.766 \pm 0.410$ & $211.2 \pm 48.6$ & -0.62 \\
NGC 6821 & 25.1 & 1 & $6.920 \pm 1.025$ & $307.4 \pm 46.1$ & -0.02 \\
\hline
\end{tabular}

Notes. The dwarf galaxies from the Dwarf Galaxy Survey are also part of the literature sample but they are not repeated here as they correspond to the sources in Table B.1. The first, second, and third columns specify the name of the source, luminosity distance, and reference to the literature work, respectively. The subsequent columns provide the GALEX FUV and MIPS $24 \mu \mathrm{m}$ or IRAS $25 \mu \mathrm{m}$ flux densities and star formation rates which are estimated from the GALEX FUV and MIPS $24 \mu \mathrm{m} / \mathrm{IRAS} 25 \mu \mathrm{m}$ measurements and the calibrations presented in Hao et al. (2011) and Kennicutt et al. (2009), respectively. ${ }^{(a)}$ Distances for galaxies with redshifts $z<0.01$ are obtained from the Nearby Galaxies Catalog (Tully 1988 ), if available, while for other galaxies distances are derived from the redshifts reported on NED. For Virgo cluster galaxies, we retrieve distances from the Goldmine database (Gavazzi et al. 2003), based on results reported in Gavazzi et al. (1999). For galaxies with redshifts $z \geq 0.01$, the luminosity distances are calculated for a spatially flat cosmology with $H_{0}=67.3 \mathrm{~km} \mathrm{~s}^{-1} \mathrm{Mpc}^{-1}, \Omega_{\lambda}=0.685$ and $\Omega_{\mathrm{m}}=0.315$ (Planck Collaboration 2014).

References. (1) Brauher et al. (2008).

Table B.4. Overview of literature data for galaxies with HII or starburst source classification used for the SFR calibrations presented in this paper.

\begin{tabular}{lrrrc}
\hline \hline Source & $\begin{array}{c}D_{\mathrm{L}}^{a} \\
{[\mathrm{Mpc}]}\end{array}$ & Ref & $\begin{array}{r}\log L_{\mathrm{TIR}} \\
{\left[\log L_{\odot}\right]}\end{array}$ & $\begin{array}{c}\log S F R \\
{\left[\log M_{\odot} \mathrm{yr}^{-1}\right]}\end{array}$ \\
\hline NGC 5713 & 33.9 & 1 & 10.91 & 1.09 \\
NGC 7714 & 41.1 & 1 & 10.75 & 0.92 \\
Cartwheel & 137.5 & 1 & 10.73 & 0.91 \\
NGC 0986 & 25.9 & 1 & 10.76 & 0.94 \\
NGC 1317 & 18.8 & 1 & 9.69 & -0.14 \\
NGC 4041 & 25.3 & 1 & 10.49 & 0.67 \\
NGC 4189 & 18.7 & 1 & 9.64 & -0.18 \\
NGC 278 & 13.2 & 1 & 10.15 & 0.33 \\
NGC 1022 & 21.7 & 1 & 10.40 & 0.57 \\
NGC 1155 & 69.8 & 1 & 10.69 & 0.86 \\
NGC 1222 & 34.5 & 1 & 10.66 & 0.83 \\
IC 1953 & 24.6 & 1 & 10.13 & 0.31 \\
NGC 1385 & 19.5 & 1 & 10.37 & 0.54 \\
UGC 02855 & 22.6 & 1 & 10.88 & 1.05 \\
NGC 1482 & 21.8 & 1 & 10.68 & 0.85 \\
NGC 1546 & 14.9 & 1 & 9.82 & -0.002 \\
ESO 317-G023 & 43.3 & 1 & 10.85 & 1.03 \\
NGC 3583 & 37.9 & 1 & 10.60 & 0.77 \\
NGC 3683 & 31.6 & 1 & 10.69 & 0.86 \\
NGC 3885 & 31.0 & 1 & 10.51 & 0.69 \\
NGC 3949 & 18.9 & 1 & 10.16 & 0.34 \\
NGC 4027 & 28.5 & 1 & 10.51 & 0.69 \\
NGC 4041 & 25.3 & 1 & 10.49 & 0.67 \\
VCC 873 & 17 & 1 & 9.82 & -0.002 \\
\hline & & & &
\end{tabular}

Notes. The first, second, and third columns specify the name of the source, luminosity distance, and reference to the literature work, respectively. The subsequent columns provide the total infrared $(8-1000 \mu \mathrm{m})$ luminosity, and star formation rate, respectively. An asterisk in Col. 1 indicates an ULIRG, and a dagger, a galaxy from a sample of 44,19 and 10 sources with [CII], [OI] $]_{63}$ and [OIII $]_{88}$ fluxes from both Herschel and ISO obervations. ${ }^{(a)}$ Distances for galaxies with redshifts $z<0.01$ are obtained from the Nearby Galaxies Catalog (Tully 1988) (if available), while for other galaxies distances are derived from the redshifts reported on NED. For Virgo cluster galaxies, we retrieve distances from the Goldmine database (Gavazzi et al. 2003), based on results reported in Gavazzi et al. (1999). For galaxies with redshifts $z \geq 0.01$, the luminosity distances are calculated for a spatially flat cosmology with $H_{0}=67.3 \mathrm{~km} \mathrm{~s}^{-1} \mathrm{Mpc}^{-1}, \Omega_{\lambda}=0.685$ and $\Omega_{\mathrm{m}}=0.315$ (Planck Collaboration 2014). ${ }^{(b)}$ The FIR line measurements correspond to the central 80" region in M 51. ${ }^{(c)}$ IRAS $15206+3342$ was observed during the Herschel performance verification phase.

References. (1) Brauher et al. (2008); (2) Parkin et al. (2013); (3) Graciá-Carpio et al. (in prep.); (4) Sargsyan et al. (2012); (5) Díaz-Santos et al. (2013); (6) Farrah et al. (2013). 
Ilse De Looze et al.: The applicability of far-infrared fine-structure lines as star formation rate tracers

Table B.4. continued.

\begin{tabular}{|c|c|c|c|c|}
\hline Source & $\begin{array}{c}D_{\mathrm{L}}^{a} \\
{[\mathrm{Mpc}]}\end{array}$ & Ref & $\begin{array}{l}\log L_{\mathrm{TIR}} \\
{\left[\log L_{\odot}\right]}\end{array}$ & $\begin{array}{c}\log S F R \\
{\left[\log M_{\odot} \mathrm{yr}^{-1}\right]}\end{array}$ \\
\hline NGC 4519 & 17 & 1 & 9.60 & -0.23 \\
\hline VCC 1972 & 17 & 1 & 9.80 & -0.03 \\
\hline VCC 1987 & 17 & 1 & 10.15 & 0.33 \\
\hline NGC 4691 & 25.1 & 1 & 10.46 & 0.63 \\
\hline IC 3908 & 19.3 & 1 & 10.00 & 0.18 \\
\hline NGC 4818 & 24.0 & 1 & 10.54 & 0.72 \\
\hline NGC 5430 & 44.3 & 1 & 10.84 & 1.01 \\
\hline NGC 5433 & 65.4 & 1 & 10.94 & 1.11 \\
\hline NGC 5937 & 42.0 & 1 & 10.77 & 0.95 \\
\hline NGC 5962 & 35.4 & 1 & 10.63 & 0.80 \\
\hline NGC 7218 & 24.5 & 1 & 10.00 & 0.18 \\
\hline NGC 7418 & 19.8 & 1 & 9.97 & 0.14 \\
\hline III Zw 107 & 86.4 & 1 & 10.57 & 0.74 \\
\hline M $51^{b}$ & 9.9 & 2 & 9.89 & 0.07 \\
\hline NGC 253 & 3.3 & 3 & 10.61 & 0.79 \\
\hline NGC 1808 & 12.0 & 3 & 10.73 & 0.91 \\
\hline NGC $3256^{\dagger}$ & 41.7 & 3 & 11.82 & 1.99 \\
\hline NGC 4945 & 5.8 & 3 & 10.92 & 1.09 \\
\hline NGC $7552^{\dagger}$ & 21.7 & 3 & 11.10 & 1.28 \\
\hline $\operatorname{Arp} 299^{\dagger}$ & 47.2 & 3 & 11.94 & 2.11 \\
\hline IRAS F17208-0014*† & 197.0 & 3 & 12.68 & 2.85 \\
\hline IRAS F20551-4250*† & 197.5 & 3 & 12.24 & 2.41 \\
\hline IRAS F23128-5919*† & 205.3 & 3 & 12.21 & 2.38 \\
\hline IRAS F10565+2448 & 198.2 & 3 & 12.28 & 2.45 \\
\hline IRAS F19297-0406*† & 405.4 & 3 & 12.61 & 2.79 \\
\hline IRAS F09022-3615* & 277.6 & 3 & 12.47 & 2.65 \\
\hline M83 center & 5.2 & 3 & 10.06 & 0.23 \\
\hline NGC 4038 & 28.3 & 3 & 10.56 & 0.74 \\
\hline IRAS $15206+3342 * \dagger c$ & 610.0 & 3 & 12.30 & 2.47 \\
\hline IRAS F22491-1808* & 366.2 & 3 & 12.42 & 2.59 \\
\hline M83 bar & 5.2 & 3 & 9.33 & -0.50 \\
\hline M83 arm & 5.2 & 3 & 8.72 & -1.11 \\
\hline NGC 4039 & 28.3 & 3 & 10.77 & 0.95 \\
\hline NGC 4038/4039 Overlap & 28.3 & 3 & 10.71 & 0.89 \\
\hline MCG-02-01-051 & 123.2 & 4 & 11.50 & 1.68 \\
\hline ESO 244-G012 & 103.8 & 4 & 11.47 & 1.65 \\
\hline UGC 01385 & 84.9 & 4 & 11.11 & 1.29 \\
\hline CGCG 052-037 & 111.2 & 4 & 11.43 & 1.61 \\
\hline ESO 286-G035 & 78.5 & 4 & 11.15 & 1.33 \\
\hline MCG-01-60-022 & 105.2 & 4 & 11.23 & 1.41 \\
\hline CGCG 381-051 & 139.9 & 4 & 11.24 & 1.42 \\
\hline NGC 6907 & 48.1 & 5 & 10.55 & 0.73 \\
\hline ESO 286-G035 & 79.0 & 5 & 10.96 & 1.14 \\
\hline ESO 343-IG013_S & 86.8 & 5 & 10.59 & 0.77 \\
\hline IRAS $21101+5810$ & 182.6 & 5 & 11.67 & 1.85 \\
\hline CGCG 453-062 & 115.3 & 5 & 11.32 & 1.50 \\
\hline Arp84_N & 52.6 & 5 & 10.86 & 1.04 \\
\hline VV705 & 186.9 & 5 & 11.84 & 2.02 \\
\hline $\mathrm{MCG}+12-02-001^{\dagger}$ & 71.2 & 5 & 11.34 & 1.52 \\
\hline UGC 12150 & 97.8 & 5 & 11.37 & 1.55 \\
\hline NGC 5331_S & 153.5 & 5 & 11.51 & 1.69 \\
\hline NGC 5331_N & 153.5 & 5 & 10.73 & 0.91 \\
\hline UGC $0838 \overline{7}^{\dagger}$ & 107.0 & 5 & 11.80 & 1.98 \\
\hline ESO 320-G030 & 48.5 & 5 & 11.34 & 1.52 \\
\hline Arp240_W & 103.8 & 5 & 10.62 & 0.80 \\
\hline Arp240_E & 103.8 & 5 & 10.86 & 1.04 \\
\hline ESO 440-IG058_S & 106.5 & 5 & 11.24 & 1.42 \\
\hline ESO 264-G057 & 78.1 & 5 & 10.86 & 1.04 \\
\hline CGCG 043-099 & 175.1 & 5 & 11.64 & 1.82 \\
\hline NGC $1614^{\dagger}$ & 72.6 & 5 & 11.50 & 1.68 \\
\hline MCG-02-33-098_W & 72.1 & 5 & 10.79 & 0.97 \\
\hline MCG-02-33-098_E & 72.1 & 5 & 10.30 & 0.48 \\
\hline NGC 5104 & 84.9 & 5 & 11.21 & 1.39 \\
\hline
\end{tabular}


Table B.4. continued.

\begin{tabular}{|c|c|c|c|c|}
\hline Source & $\begin{array}{c}D_{\mathrm{L}}^{a} \\
{[\mathrm{Mpc}]}\end{array}$ & Ref & $\begin{array}{l}\log L_{\mathrm{TIR}} \\
{\left[\log L_{\odot}\right]}\end{array}$ & $\begin{array}{c}\log S F R \\
{\left[\log M_{\odot} \mathrm{yr}^{-1}\right]}\end{array}$ \\
\hline ESO 267-G030_W & 84.5 & 5 & 10.96 & 1.14 \\
\hline ESO 267-G030_E & 84.5 & 5 & 10.90 & 1.08 \\
\hline ESO $173-\mathrm{G} 015^{\dagger}$ & 44.0 & 5 & 11.42 & 1.60 \\
\hline NGC 6621 & 94.6 & 5 & 11.24 & 1.42 \\
\hline NGC 6090 & 138.1 & 5 & 11.44 & 1.62 \\
\hline CGCG 049-057 & 58.9 & 5 & 11.20 & 1.38 \\
\hline IRAS 13052-5711 & 97.3 & 5 & 11.17 & 1.35 \\
\hline ESO 255-IG007_E & 181.7 & 5 & 10.74 & 0.92 \\
\hline ESO 255-IG007_W & 181.7 & 5 & 11.75 & 1.93 \\
\hline ESO 255-IG007_S & 181.7 & 5 & 10.75 & 0.93 \\
\hline IRAS F05187-1017 & 130.6 & 5 & 11.40 & 1.58 \\
\hline IRAS F03217+4022 & 107.5 & 5 & 11.42 & 1.60 \\
\hline ESO 069-IG006 * & 219.6 & 5 & 12.05 & 2.23 \\
\hline NGC 2342 & 80.4 & 5 & 10.89 & 1.07 \\
\hline NGC 2388 & 62.6 & 5 & 11.23 & 1.41 \\
\hline IC 4734 & 70.8 & 5 & 11.33 & 1.51 \\
\hline UGC 11041 & 74.0 & 5 & 10.82 & 1.00 \\
\hline IRAS 18090+0130_W & 133.4 & 5 & 10.98 & 1.16 \\
\hline IRAS 18090+0130_E & 133.4 & 5 & 11.47 & 1.65 \\
\hline VIIZw031* & 249.8 & 5 & 12.03 & 2.21 \\
\hline NGC 6286_N & 83.6 & 5 & 10.51 & 0.69 \\
\hline NGC 6286_S & 83.6 & 5 & 11.01 & 1.19 \\
\hline CGCG 142-034_E & 85.4 & 5 & 10.84 & 1.02 \\
\hline UGC 04881_W & 185.0 & 5 & 10.86 & 1.04 \\
\hline UGC 03410_W & 59.4 & 5 & 9.84 & 0.02 \\
\hline UGC 03410_E & 59.4 & 5 & 10.51 & 0.69 \\
\hline ESO 557-G002_S & 97.3 & 5 & 10.40 & 0.58 \\
\hline ESO 557-G002_N & 97.3 & 5 & 11.16 & 1.34 \\
\hline ESO 432-IG006_W & 73.5 & 5 & 10.70 & 0.92 \\
\hline UGC 03608 & 97.8 & 5 & 11.20 & 1.38 \\
\hline IRAS F18293-3413 & 82.7 & 5 & 11.85 & 2.03 \\
\hline VV414_W & 115.3 & 5 & 10.93 & 1.11 \\
\hline NGC 3110 & 76.7 & 5 & 10.95 & 1.13 \\
\hline Arp303_S & 91.4 & 5 & 10.51 & 0.69 \\
\hline Arp303_N & 91.4 & 5 & 10.39 & 0.57 \\
\hline ESO 593-IG008 & 231.1 & 5 & 11.87 & 2.05 \\
\hline ESO 467-G027 & 79.4 & 5 & 10.47 & 0.65 \\
\hline CGCG 247-020 & 119.5 & 5 & 11.35 & 1.53 \\
\hline ESO 077-IG014_W & 195.4 & 5 & 11.11 & 1.29 \\
\hline IC 5179 & 51.7 & 5 & 10.77 & 0.95 \\
\hline MCG+04-48-002 & 63.0 & 5 & 10.88 & 1.06 \\
\hline IRAS $20351+2521$ & 156.7 & 5 & 11.45 & 1.63 \\
\hline VV250a_W & 144.1 & 5 & 10.87 & 1.05 \\
\hline VV250a_E & 144.1 & 5 & 11.70 & 1.88 \\
\hline UGC 08739 & 76.2 & 5 & 10.52 & 0.70 \\
\hline NGC $4194^{\dagger}$ & 43.6 & 5 & 10.94 & 1.12 \\
\hline NGC 7592_E & 112.1 & 5 & 11.10 & 1.28 \\
\hline NGC 6670_W & 132.0 & 5 & 11.21 & 1.39 \\
\hline NGC 6670_E & 132.0 & 5 & 11.27 & 1.45 \\
\hline NGC 3221 & 62.1 & 5 & 9.93 & 0.11 \\
\hline MCG+07-23-019 & 160.5 & 5 & 11.64 & 1.82 \\
\hline Arp256 & 125.5 & 5 & 11.44 & 1.62 \\
\hline VV340a_S & 156.7 & 5 & 10.59 & 0.77 \\
\hline VV340a_N & 156.7 & 5 & 11.37 & 1.55 \\
\hline NGC 0023 & 68.9 & 5 & 10.93 & 1.11 \\
\hline NGC 6701 & 59.8 & 5 & 10.65 & 0.83 \\
\hline MCG-03-04-014 & 155.8 & 5 & 11.63 & 1.81 \\
\hline NGC 7679 & 78.1 & 5 & 10.88 & 1.06 \\
\hline UGC 01385 & 85.4 & 5 & 11.03 & 1.21 \\
\hline NGC $6052^{\dagger}$ & 71.7 & 5 & 10.52 & 0.70 \\
\hline ESO 244-G012 & 96.4 & 5 & 11.40 & 1.58 \\
\hline ESO 507-G070 & 99.2 & 5 & 11.59 & 1.77 \\
\hline
\end{tabular}


Ilse De Looze et al.: The applicability of far-infrared fine-structure lines as star formation rate tracers

Table B.4. continued.

\begin{tabular}{|c|c|c|c|c|}
\hline Source & $\begin{array}{c}D_{\mathrm{L}}^{a} \\
{[\mathrm{Mpc}]}\end{array}$ & Ref & $\begin{array}{l}\log L_{\mathrm{TIR}} \\
{\left[\log L_{\odot}\right]}\end{array}$ & $\begin{array}{c}\log S F R \\
{\left[\log M_{\odot} \mathrm{yr}^{-1}\right]}\end{array}$ \\
\hline IC 4280 & 74.0 & 5 & 10.65 & 0.83 \\
\hline Arp86_S & 77.6 & 5 & 10.50 & 0.68 \\
\hline NGC 0232_W & 101.5 & 5 & 11.47 & 1.65 \\
\hline NGC 7771_N & 64.8 & 5 & 11.29 & 1.47 \\
\hline $\operatorname{Mrk} 331^{\dagger}$ & 84.5 & 5 & 11.56 & 1.74 \\
\hline NGC 5936 & 60.3 & 5 & 10.79 & 0.97 \\
\hline NGC 5653 & 53.9 & 5 & 10.59 & 0.77 \\
\hline ESO 099-G004 & 135.3 & 5 & 11.67 & 1.85 \\
\hline ESO 221-IG010 & 46.7 & 5 & 10.66 & 0.84 \\
\hline NGC $695^{\dagger}$ & 150.7 & 6 & 11.49 & 1.67 \\
\hline MCG+05-06-036_S & 156.7 & 5 & 11.50 & 1.67 \\
\hline UGC 02369 & 147.9 & 5 & 11.66 & 1.84 \\
\hline UGC $02238^{\dagger}$ & 100.1 & 5 & 11.26 & 1.44 \\
\hline ESO 353-G020 & 72.6 & 5 & 11.08 & 1.26 \\
\hline NGC 828 & 81.7 & 5 & 11.19 & 1.37 \\
\hline IRAS F16164-0746 & 125.0 & 5 & 11.68 & 1.86 \\
\hline NGC 0838_E & 58.0 & 5 & 10.92 & 1.10 \\
\hline NGC 0838_S & 58.0 & 5 & 11.04 & 1.22 \\
\hline UGC 01845 & 70.8 & 5 & 11.10 & 1.28 \\
\hline IC 0214 & 139.9 & 5 & 11.16 & 1.34 \\
\hline NGC 992 & 62.6 & 5 & 10.77 & 0.95 \\
\hline CGCG 465-012_N & 101.9 & 5 & 10.43 & 0.61 \\
\hline CGCG 465-012_S & 101.9 & 5 & 11.06 & 1.24 \\
\hline MCG-05-12-006 & 85.4 & 5 & 11.06 & 1.24 \\
\hline CGCG 052-037 & 112.5 & 5 & 11.32 & 1.50 \\
\hline IRAS F16516-0948 & 103.3 & 5 & 10.96 & 1.14 \\
\hline UGC 02982 & 80.8 & 5 & 10.95 & 1.13 \\
\hline IC 4687 & 79.0 & 5 & 11.21 & 1.39 \\
\hline IRAS 17578-0400_N & 63.5 & 5 & 11.45 & 1.63 \\
\hline ESO 138-G027 & 95.0 & 5 & 11.26 & 1.44 \\
\hline IRAS 05083+2441_S & 105.6 & 5 & 11.16 & 1.34 \\
\hline IRAS $04271+3849$ & 85.9 & 5 & 11.04 & 1.22 \\
\hline NGC 1797 & 67.1 & 5 & 11.01 & 1.19 \\
\hline IRAS F17138-1017 & 79.0 & 5 & 11.35 & 1.53 \\
\hline CGCG 468-002_E & 83.1 & 5 & 11.17 & 1.35 \\
\hline IRAS $05442+1732$ & 84.9 & 5 & 11.21 & 1.39 \\
\hline MCG+08-11-002 & 87.2 & 5 & 11.47 & 1.65 \\
\hline IRAS $05129+5128$ & 126.4 & 5 & 11.33 & 1.51 \\
\hline UGC 03351 & 67.6 & 5 & 11.22 & 1.40 \\
\hline NGC $2146^{\dagger}$ & 19.2 & 5 & 10.83 & 1.01 \\
\hline NGC 317B & 82.7 & 5 & 11.32 & 1.50 \\
\hline AM0702-601_S & 145.1 & 5 & 11.37 & 1.55 \\
\hline RR032_N & 79.0 & 5 & 10.71 & 0.89 \\
\hline RR032_S & 79.0 & 5 & 10.73 & 0.91 \\
\hline IRAS F11231+1456 & 158.1 & 5 & 11.55 & 1.73 \\
\hline IRAS 01003-2238* & 571.0 & 6 & 12.32 & 2.50 \\
\hline IRAS 20100-4156*广 & 633.9 & 6 & 12.67 & 2.85 \\
\hline IRAS 00397-1312* & 1378.7 & 6 & 12.90 & 3.08 \\
\hline IRAS 06035-7102* & 372.5 & 6 & 12.22 & 2.40 \\
\hline IRAS 20414-1651* & 412.5 & 6 & 12.22 & 2.40 \\
\hline IRAS 23253-5415* & 633.9 & 6 & 12.36 & 2.54 \\
\hline
\end{tabular}


Table B.5. Overview of literature data for galaxies with composite or AGN source classification used for the SFR calibrations presented in this paper.

\begin{tabular}{|c|c|c|c|c|}
\hline Source & $\begin{array}{c}D_{\mathrm{L}}^{a} \\
{[\mathrm{Mpc}]}\end{array}$ & Ref & $\begin{array}{l}\log L_{\mathrm{TIR}} \\
{\left[\log L_{\odot}\right]}\end{array}$ & $\begin{array}{c}\log S F R \\
{\left[\log M_{\odot} \mathrm{yr}^{-1}\right]}\end{array}$ \\
\hline NGC 0660 & 13.2 & 1 & 10.53 & 0.70 \\
\hline NGC 1266 & 32.8 & 1 & 10.55 & 0.73 \\
\hline NGC 4278 & 10.8 & 1 & 8.59 & -1.24 \\
\hline NGC 4293 & 18.9 & 1 & 9.74 & -0.09 \\
\hline NGC 4651 & 18.7 & 1 & 9.87 & 0.04 \\
\hline NGC 4698 & 18.7 & 1 & 9.00 & -0.83 \\
\hline NGC 6221 & 21.6 & 1 & 10.80 & 0.98 \\
\hline NGC 6810 & 28.2 & 1 & 10.70 & 0.88 \\
\hline NGC 7217 & 17.8 & 1 & 9.83 & 0.003 \\
\hline NGC 449 & 71.9 & 1 & 10.74 & 0.91 \\
\hline Mrk 573 & 77.5 & 1 & 10.58 & 0.76 \\
\hline NGC 1052 & 22.5 & 1 & 9.30 & -0.52 \\
\hline NGC 1326 & 18.8 & 1 & 9.95 & 0.12 \\
\hline IC 356 & 18.1 & 1 & 9.85 & 0.02 \\
\hline IC 450 & 85.0 & 1 & 10.66 & 0.83 \\
\hline NGC 3705 & 18.9 & 1 & 9.75 & -0.07 \\
\hline NGC 4102 & 18.9 & 1 & 10.71 & 0.88 \\
\hline NGC 4314 & 17 & 1 & 9.53 & -0.30 \\
\hline VCC 857 & 17 & 1 & 9.17 & -0.65 \\
\hline NGC 4414 & 17 & 1 & 10.45 & 0.62 \\
\hline VCC 1003 & 17 & 1 & 9.31 & -0.52 \\
\hline VCC 1043 & 17 & 1 & 9.60 & -0.23 \\
\hline VCC 1110 & 17 & 1 & 9.32 & -0.51 \\
\hline NGC 4486 & 17 & 1 & 9.00 & -0.82 \\
\hline VCC 1727 & 17 & 1 & 9.78 & -0.05 \\
\hline NGC 5643 & 18.8 & 1 & 10.38 & 0.56 \\
\hline IZw 92 & 173.2 & 1 & 11.20 & 1.37 \\
\hline NGC 5866 & 17.1 & 1 & 9.79 & -0.04 \\
\hline NGC 5953 & 36.8 & 1 & 10.64 & 0.82 \\
\hline NGC 6217 & 26.6 & 1 & 10.39 & 0.57 \\
\hline NGC 6574 & 39.0 & 1 & 10.84 & 1.01 \\
\hline NGC 6764 & 41.2 & 1 & 10.57 & 0.75 \\
\hline Mrk 509 & 157.2 & 1 & 11.25 & 1.42 \\
\hline NGC 6958 & 39.5 & 1 & 9.82 & -0.003 \\
\hline IC 5063 & 51.0 & 1 & 10.88 & 1.06 \\
\hline IC 1459 & 22.3 & 1 & 9.12 & -0.71 \\
\hline NGC 1365 & 18.8 & 2 & 11.09 & 1.26 \\
\hline NGC 3783 & 42.9 & 2 & 10.27 & 0.45 \\
\hline NGC 4051 & 18.9 & 2 & 9.63 & -0.19 \\
\hline NGC $4151^{\dagger}$ & 22.6 & 2 & 9.95 & 0.12 \\
\hline NGC 4593 & 44.0 & 2 & 10.34 & 0.50 \\
\hline NGC 5033 & 20.8 & 2 & 10.44 & 0.61 \\
\hline NGC 5506 & 32.0 & 2 & 10.44 & 0.61 \\
\hline NGC $7469^{\dagger}$ & 74.2 & 2 & 11.70 & 1.88 \\
\hline IC 4329A $A^{\dagger}$ & 72.0 & 2 & 10.59 & 0.76 \\
\hline Cen A & 5.5 & 2 & 9.98 & 0.16 \\
\hline Circinus & 4.7 & 2 & 10.39 & 0.57 \\
\hline NGC $1068^{\dagger}$ & 16.0 & 2 & 11.26 & 1.43 \\
\hline NGC $1275^{\dagger}$ & 79.0 & 2 & 11.19 & 1.37 \\
\hline NGC 1386 & 18.8 & 2 & 9.89 & 0.07 \\
\hline
\end{tabular}

Notes. The first, second, and third columns specify the name of the source, luminosity distance, and reference to the literature work, respectively. The subsequent columns provide the total infrared $(8-1000 \mu \mathrm{m})$ luminosity and star formation rate, respectively. Asterisks and daggers are the same as in Table B.4. ${ }^{(a)}$ Distances for galaxies with redshifts $z<0.01$ are obtained from the Nearby Galaxies Catalog (Tully 1988) (if available), while for other galaxies distances are derived from the redshifts reported on NED. For Virgo cluster galaxies, we retrieve distances from the Goldmine database (Gavazzi et al. 2003), based on results reported in Gavazzi et al. (1999). For galaxies with redshifts $z \geq 0.01$, the luminosity distances are calculated for a spatially flat cosmology with $H_{0}=67.3 \mathrm{~km} \mathrm{~s}^{-1} \mathrm{Mpc}^{-1}, \Omega_{\lambda}=0.685$ and $\Omega_{\mathrm{m}}=0.315$ (Planck Collaboration XVI 2014). ${ }^{(b)}$ IRAS F07251-0248 did not have an optical source classification in Graciá-Carpio et al. (in prep.). By relying on the equivalent width of the $6.2 \mu \mathrm{m}$ PAH feature $\left(E W(\right.$ PAH $6.2 \mu \mathrm{m})=0.29$, Stierwalt et al. 2013), we classify the source as an AGN. ${ }^{(c)}$ IRAS F19542-1110 did not have an optical source classification in Graciá-Carpio et al. (in prep.). By relying on the equivalent width of the $6.2 \mu \mathrm{m}$ PAH feature $(E W($ PAH $6.2 \mu \mathrm{m})=0.09$, Stierwalt et al. 2013), we classify the source as an AGN.

References. (1) Brauher et al. (2008); (2) Graciá-Carpio et al. (in prep.); (3) Sargsyan et al. (2012); (4) Díaz-Santos et al. (2013); (5) Farrah et al. (2013). 
Ilse De Looze et al.: The applicability of far-infrared fine-structure lines as star formation rate tracers

Table B.5. continued.

\begin{tabular}{|c|c|c|c|c|}
\hline Source & $\begin{array}{c}D_{\mathrm{L}}^{a} \\
{[\mathrm{Mpc}]}\end{array}$ & Ref & $\begin{array}{l}\log L_{\mathrm{TIR}} \\
{\left[\log L_{\odot}\right]}\end{array}$ & $\begin{array}{c}\log S F R \\
{\left[\log M_{\odot} \mathrm{yr}^{-1}\right]}\end{array}$ \\
\hline NGC 7314 & 20.4 & 2 & 9.61 & -0.21 \\
\hline NGC $7582^{\dagger}$ & 19.6 & 2 & 10.82 & 1.00 \\
\hline $\operatorname{Mrk} 3^{\dagger}$ & 60.8 & 2 & 10.60 & 0.77 \\
\hline IRAS F18325-5926 & 90.0 & 2 & 10.89 & 1.06 \\
\hline UGC 5101* & 180.8 & 2 & 12.17 & 2.35 \\
\hline $\operatorname{Mrk} 231 * \dagger$ & 193.9 & 2 & 12.62 & 2.79 \\
\hline Mrk 273*广 & 173.0 & 2 & 12.36 & 2.53 \\
\hline NGC 6240*† & 112.3 & 2 & 12.01 & 2.19 \\
\hline IRAS F23365+3604*广 & 301.1 & 2 & 12.37 & 2.55 \\
\hline IRAS F15250+3609* & 257.0 & 2 & 12.19 & 2.37 \\
\hline IRAS F12112+0305* & 342.7 & 2 & 12.48 & 2.65 \\
\hline IRAS F13120-5453* & 141.6 & 2 & 12.47 & 2.64 \\
\hline IRAS F14348-1447* & 390.3 & 2 & 12.60 & 2.77 \\
\hline IRAS F05189-2524*广 & 196.6 & 2 & 12.25 & 2.43 \\
\hline IRAS F08572+3915* & 270.7 & 2 & 12.34 & 2.52 \\
\hline NGC 3079 & 22.73 & 2 & 10.95 & 1.13 \\
\hline M82 center & 5.8 & 2 & 11.28 & 1.45 \\
\hline IRAS F07251-0248*b & 277.6 & 2 & 12.47 & 2.65 \\
\hline IRAS F19542-1110*c & 292.0 & 2 & 12.21 & 2.39 \\
\hline NGC $4418^{\dagger}$ & 31.9 & 2 & 11.26 & 1.43 \\
\hline $\operatorname{Arp} 220^{* \dagger}$ & 81.4 & 2 & 12.46 & 2.64 \\
\hline IRAS F14378-3651* & 318.9 & 2 & 12.33 & 2.51 \\
\hline Mrk 334 & 99.2 & 3 & 11.16 & 1.34 \\
\hline IRAS00199-7426* & 459.9 & 3 & 12.37 & 2.55 \\
\hline E12-G21 & 150.7 & 3 & 11.10 & 1.25 \\
\hline IRAS F00456-2904SW* & 531.1 & 3 & 12.26 & 2.44 \\
\hline MCG-03-04-014 & 160.0 & 3 & 11.67 & 1.85 \\
\hline NGC 454 & 54.8 & 3 & 10.31 & 0.49 \\
\hline ESO 353-G020 & 71.7 & 3 & 11.13 & 1.31 \\
\hline IRAS F01364-1042 & 222.5 & 3 & 11.85 & 2.03 \\
\hline NGC 788 & 61.2 & 3 & 10.21 & 0.39 \\
\hline Mrk 590 & 111.6 & 3 & 10.74 & 0.92 \\
\hline UGC 01845 & 70.3 & 3 & 11.17 & 1.35 \\
\hline IC 1816 & 76.2 & 3 & 10.56 & 0.74 \\
\hline NGC 973 & 73.1 & 3 & 10.51 & 0.69 \\
\hline IRAS F02437+2122 & 105.6 & 3 & 11.22 & 1.40 \\
\hline UGC 02369 & 142.3 & 3 & 11.67 & 1.85 \\
\hline Mrk 1066 & 53.9 & 3 & 10.97 & 1.15 \\
\hline IRAS F03217+4022 & 106.1 & 3 & 11.37 & 1.55 \\
\hline Mrk 609 & 157.7 & 3 & 11.44 & 1.62 \\
\hline IRAS F03359+1523 & 161.9 & 3 & 11.62 & 1.80 \\
\hline IRAS F03450+0055 & 141.3 & 3 & 11.09 & 1.27 \\
\hline IRAS 04103-2838* & 568.4 & 3 & 12.25 & 2.43 \\
\hline IRAS 04114-5117* & 607.5 & 3 & 12.29 & 2.47 \\
\hline ESO 420-G013 & 53.5 & 3 & 11.15 & 1.33 \\
\hline $3 \mathrm{C} 120$ & 150.7 & 3 & 11.23 & 1.41 \\
\hline ESO 203-IG001 & 245.0 & 3 & 11.90 & 2.08 \\
\hline MCG-05-12-006 & 84.9 & 3 & 11.24 & 1.42 \\
\hline Ark120 & 149.3 & 3 & 11.07 & 1.25 \\
\hline IRAS F05187-1017 & 128.8 & 3 & 11.33 & 1.50 \\
\hline 2MASXJ05580206-3820043 & 154.9 & 3 & 11.23 & 1.41 \\
\hline IRAS F06076-2139 & 171.3 & 3 & 11.68 & 1.86 \\
\hline IRAS 06301-7934* & 775.2 & 3 & 12.41 & 2.59 \\
\hline IRAS 06361-6217* & 792.6 & 3 & 12.46 & 2.63 \\
\hline NGC 2273 & 31.6 & 3 & 10.35 & 0.53 \\
\hline UGC 03608 & 96.9 & 3 & 11.24 & 1.42 \\
\hline IRAS F06592-6313 & 104.2 & 3 & 11.24 & 1.42 \\
\hline Mrk 9 & 183.1 & 3 & 11.20 & 1.37 \\
\hline IRAS $07598+6508 *$ & 734.1 & 3 & 12.56 & 2.74 \\
\hline Mrk 622 & 105.2 & 3 & 10.77 & 0.95 \\
\hline ESO 60-IG016 & 209.6 & 3 & 11.77 & 1.95 \\
\hline Mrk 18 & 49.9 & 3 & 10.22 & 0.40 \\
\hline
\end{tabular}


Table B.5. continued.

\begin{tabular}{|c|c|c|c|c|}
\hline Source & $\begin{array}{c}D_{\mathrm{L}}^{a} \\
{[\mathrm{Mpc}]}\end{array}$ & Ref & $\begin{array}{l}\log L_{\mathrm{TIR}} \\
{\left[\log L_{\odot}\right]}\end{array}$ & $\begin{array}{c}\log S F R \\
{\left[\log M_{\odot} \mathrm{yr}^{-1}\right]}\end{array}$ \\
\hline MCG-01-24-012 & 88.6 & 3 & 10.43 & 0.61 \\
\hline Mrk 705 & 132.9 & 3 & 10.75 & 0.93 \\
\hline IRAS F10038-3338 & 156.3 & 3 & 11.74 & 1.92 \\
\hline NGC 3393 & 56.2 & 3 & 10.47 & 0.65 \\
\hline ESO 319-G022 & 74.0 & 3 & 11.07 & 1.25 \\
\hline IRAS12018+1941* & 841.8 & 3 & 12.55 & 2.73 \\
\hline UGC 07064 & 113.5 & 3 & 11.09 & 1.27 \\
\hline NGC 4507 & 53.0 & 3 & 10.74 & 0.92 \\
\hline PG1244+026 & 222.5 & 3 & 11.05 & 1.23 \\
\hline ESO 507-G070 & 98.3 & 3 & 11.51 & 1.69 \\
\hline NGC 4941 & 7.1 & 3 & 8.87 & -0.95 \\
\hline ESO 323-G077 & 70.3 & 3 & 11.03 & 1.21 \\
\hline MCG-03-34-064 & 74.4 & 3 & 11.19 & 1.37 \\
\hline IRAS F13279+3401 & 107.9 & 3 & 10.53 & 0.71 \\
\hline$M-6-30-15$ & 34.5 & 3 & 10.04 & 0.22 \\
\hline IRAS $13342+3932 *$ & 900.9 & 3 & 12.49 & 2.67 \\
\hline IRAS F13349+2438* & 517.1 & 3 & 12.35 & 2.53 \\
\hline NGC 5347 & 40.9 & 3 & 10.26 & 0.44 \\
\hline $\mathrm{OQ}+208$ & 360.6 & 3 & 11.72 & 1.90 \\
\hline NGC 5548 & 77.6 & 3 & 10.68 & 0.86 \\
\hline Mrk 1490 & 116.7 & 3 & 11.37 & 1.55 \\
\hline PG1426+015 & 410.0 & 3 & 11.55 & 1.73 \\
\hline PG1440+356 & 367.6 & 3 & 11.57 & 1.75 \\
\hline NGC 5728 & 47.0 & 3 & 10.77 & 0.95 \\
\hline NGC 5793 & 52.1 & 3 & 10.72 & 0.90 \\
\hline IRAS $15001+1433 *$ & 809.5 & 3 & 12.52 & 2.70 \\
\hline IRAS $15225+2350^{*}$ & 681.6 & 3 & 12.21 & 2.39 \\
\hline Mrk 876* & 628.6 & 3 & 12.01 & 2.19 \\
\hline IRAS F16164-0746 & 106.5 & 3 & 11.62 & 1.80 \\
\hline Mrk 883 & 173.2 & 3 & 11.01 & 1.19 \\
\hline IRAS 16334+4630* & 965.2 & 3 & 12.49 & 2.67 \\
\hline ESO 069-IG006 & 213.9 & 3 & 11.98 & 2.16 \\
\hline IRAS F16399-0937N & 122.7 & 3 & 11.55 & 1.73 \\
\hline IRAS $17044+6720^{*}$ & 660.3 & 3 & 12.21 & 2.39 \\
\hline IRAS $17068+4027 *$ & 899.3 & 3 & 12.44 & 2.62 \\
\hline IRAS F17132+5313 & 235.4 & 3 & 11.94 & 2.12 \\
\hline ESO 138-G027 & 94.1 & 3 & 11.41 & 1.59 \\
\hline CGCG 141-034 & 85.9 & 3 & 11.18 & 1.36 \\
\hline IC 4734 & 70.3 & 3 & 11.35 & 1.53 \\
\hline IRAS18443+7433* & 658.7 & 3 & 12.37 & 2.55 \\
\hline ESO 140-G043 & 63.9 & 3 & 10.65 & 0.83 \\
\hline H1846-786 & 348.3 & 3 & 11.46 & 1.64 \\
\hline ESO 593-IG008 & 224.9 & 3 & 11.94 & 2.12 \\
\hline ESO-141-G055 & 169.9 & 3 & 11.11 & 1.29 \\
\hline ESO 339-G011 & 86.8 & 3 & 11.18 & 1.36 \\
\hline IRAS $20037-1547 *$ & 971.4 & 3 & 12.66 & 2.84 \\
\hline NGC 6860 & 67.1 & 3 & 10.44 & 0.62 \\
\hline NGC 7213 & 24.5 & 3 & 10.10 & 0.28 \\
\hline ESO 602-G025 & 113.5 & 3 & 11.39 & 1.57 \\
\hline UGC 12138 & 113.5 & 3 & 10.82 & 1.00 \\
\hline UGC 12150 & 96.9 & 3 & 11.38 & 1.56 \\
\hline ESO 239-IG002 & 197.7 & 3 & 11.85 & 2.03 \\
\hline Zw453.062 & 113.9 & 3 & 11.41 & 1.59 \\
\hline NGC 7603 & 134.3 & 3 & 10.90 & 1.08 \\
\hline ESO 339-G011 & 87.7 & 4 & 11.00 & 1.18 \\
\hline NGC 6926 & 89.5 & 4 & 10.34 & 0.52 \\
\hline ESO 343-IG013_N & 86.8 & 4 & 10.86 & 1.04 \\
\hline CGCG 448-020_E & 168.5 & 4 & 11.78 & 1.96 \\
\hline CGCG 448-020c & 168.5 & 4 & 10.66 & 0.84 \\
\hline IC $1623 \mathrm{~B}$ & 91.8 & 4 & 11.47 & 1.65 \\
\hline IC $2810 b$ & 158.1 & 4 & 11.27 & 1.45 \\
\hline NGC 4922 & 108.4 & 4 & 11.28 & 1.46 \\
\hline
\end{tabular}


Ilse De Looze et al.: The applicability of far-infrared fine-structure lines as star formation rate tracers

Table B.5. continued.

\begin{tabular}{|c|c|c|c|c|}
\hline Source & $\begin{array}{c}D_{\mathrm{L}}^{a} \\
{[\mathrm{Mpc}]}\end{array}$ & Ref & $\begin{array}{l}\log L_{\mathrm{TIR}} \\
{\left[\log L_{\odot}\right]}\end{array}$ & $\begin{array}{c}\log S F R \\
{\left[\log M_{\odot} \mathrm{yr}^{-1}\right]}\end{array}$ \\
\hline CGCG 011-0767 & 114.4 & 4 & 11.26 & 1.44 \\
\hline IRAS23436+5257 & 158.6 & 4 & 11.54 & 1.72 \\
\hline ESO 264-G036 & 96.0 & 4 & 10.97 & 1.15 \\
\hline NGC 5135 & 62.1 & 4 & 11.33 & 1.51 \\
\hline MCG-03-34-064 & 75.3 & 4 & 10.85 & 1.03 \\
\hline IRAS08355-4944 & 119.0 & 4 & 11.39 & 1.57 \\
\hline ESO 203-IG001 & 252.2 & 4 & 11.91 & 2.09 \\
\hline IRAS12116-5615 & 125.0 & 4 & 11.63 & 1.81 \\
\hline IRASF06076-2139 & 175.1 & 4 & 11.67 & 1.85 \\
\hline NGC 2342b & 80.4 & 4 & 11.02 & 1.20 \\
\hline NGC 2388b & 62.6 & 4 & 9.93 & 0.11 \\
\hline NGC 2623 & 84.5 & 4 & 11.71 & 1.89 \\
\hline CGCG 142-034_W & 85.4 & 4 & 10.44 & 0.62 \\
\hline UGC 04881_E & 185.0 & 4 & 11.58 & 1.76 \\
\hline MCG+02-20-003_N & 74.0 & 4 & 11.07 & 1.25 \\
\hline NGC 1961 & 59.4 & 4 & 9.66 & -0.16 \\
\hline ESO 432-IG006_E & 73.5 & 4 & 10.75 & 0.93 \\
\hline NGC 2369 & 49.0 & 4 & 11.11 & 1.29 \\
\hline IRASF06592-6313 & 105.2 & 4 & 11.34 & 1.52 \\
\hline IRASF17132+5313 & 242.1 & 4 & 11.75 & 1.93 \\
\hline VV414_E & 115.3 & 4 & 11.01 & 1.19 \\
\hline ESO 60-IG016 & 218.7 & 4 & 11.67 & 1.86 \\
\hline IRASF10173+0828 & 233.0 & 4 & 11.89 & 2.07 \\
\hline NGC 5256 & 128.3 & 4 & 11.30 & 1.48 \\
\hline MCG+08-18-013 & 119.5 & 4 & 11.35 & 1.53 \\
\hline ESO 077-IG014_E & 195.4 & 4 & 11.68 & 1.86 \\
\hline NGC 7592_W & 112.1 & 4 & 10.32 & 0.50 \\
\hline NGC 7674 & 133.9 & 4 & 11.24 & 1.42 \\
\hline IRASF12224-0624 & 121.3 & 4 & 11.41 & 1.59 \\
\hline IC 0860 & 50.3 & 4 & 11.08 & 1.26 \\
\hline ESO 239-IG002 & 202.5 & 4 & 11.90 & 2.08 \\
\hline NGC 7591 & 75.3 & 4 & 11.07 & 1.25 \\
\hline MCG-01-60-022 & 106.5 & 4 & 11.12 & 1.30 \\
\hline IRASF01364-1042 & 228.7 & 4 & 11.86 & 2.04 \\
\hline NGC 34 & 89.5 & 4 & 11.50 & 1.68 \\
\hline ESO 319-G022 & 74.4 & 4 & 11.01 & 1.19 \\
\hline IC 5298 & 126.4 & 4 & 11.54 & 1.72 \\
\hline Arp86_N & 77.6 & 4 & 9.94 & 0.12 \\
\hline NGC 5734_N & 62.1 & 4 & 10.78 & 0.96 \\
\hline NGC 5734_S & 62.1 & 4 & 10.43 & 0.61 \\
\hline NGC 5990 & 58.0 & 4 & 10.81 & 0.99 \\
\hline NGC 0232_E & 101.5 & 4 & 10.75 & 0.93 \\
\hline NGC 77714_W & 64.8 & 4 & 10.21 & 0.39 \\
\hline ESO 420-G013 & 53.9 & 4 & 10.98 & 1.16 \\
\hline MCG+05-06-036_N & 156.7 & 4 & 10.98 & 1.16 \\
\hline CGCG 436-030 & 144.6 & 4 & 11.67 & 1.85 \\
\hline IIIZw035 & 128.8 & 4 & 11.66 & 1.84 \\
\hline NGC $6156^{\dagger}$ & 49.0 & 4 & 10.57 & 0.75 \\
\hline NGC 0838_W & 58.0 & 4 & 10.53 & 0.71 \\
\hline NGC 0958 & 87.2 & 4 & 10.00 & 0.18 \\
\hline NGC 0877_S & 58.9 & 4 & 10.34 & 0.52 \\
\hline NGC 0877_N & 58.9 & 4 & 9.98 & 0.16 \\
\hline UGC 02608_N & 107.0 & 4 & 11.25 & 1.43 \\
\hline IRASF03359+1523 & 165.2 & 4 & 11.59 & 1.77 \\
\hline NGC 1572 & 93.2 & 4 & 11.39 & 1.57 \\
\hline IRASF02437+2122 & 107.0 & 4 & 11.26 & 1.44 \\
\hline ESO 550-IG025a & 148.8 & 4 & 11.36 & 1.54 \\
\hline ESO 550-IG025b & 148.8 & 4 & 11.09 & 1.27 \\
\hline IC $4687 b$ & 79.0 & 4 & 10.56 & 0.74 \\
\hline IC $4687 \mathrm{c}$ & 79.0 & 4 & 10.84 & 1.02 \\
\hline IC 4518ABa & 72.1 & 4 & 10.79 & 0.97 \\
\hline UGC 03094 & 113.5 & 4 & 11.12 & 1.30 \\
\hline IRASF16399-0937 & 124.6 & 4 & 11.58 & 1.76 \\
\hline
\end{tabular}


A\&A 568, A62 (2014)

Table B.5. continued.

\begin{tabular}{lrrrc}
\hline \hline Source & $\begin{array}{c}D_{\mathrm{L}}^{a} \\
{[\mathrm{Mpc}]}\end{array}$ & Ref & $\begin{array}{r}\log L_{\mathrm{TIR}} \\
{\left[\log L_{\odot}\right]}\end{array}$ & $\begin{array}{c}\log S F R \\
{\left[\log M_{\odot} \mathrm{yr}^{-1}\right]}\end{array}$ \\
\hline CGCG 141-034 & 90.4 & 4 & 11.19 & 1.37 \\
CGCG 468-002_W & 83.1 & 4 & 10.54 & 0.72 \\
IRAS03582+6012_E & 139.0 & 4 & 11.13 & 1.31 \\
ESO 602-G025 & 115.3 & 4 & 11.31 & 1.49 \\
ESO 453-G005_S & 95.0 & 4 & 11.36 & 1.54 \\
AM0702-601_N & 145.1 & 4 & 11.14 & 1.32 \\
IRAS 08311-2459* & 478.2 & 5 & 12.50 & 2.68 \\
IRAS 11095-0238* & 514.1 & 5 & 12.28 & 2.46 \\
IRAS 23230-6926* & 514.1 & 5 & 12.37 & 2.55 \\
3C 273* & 783.9 & 5 & 12.83 & 3.01 \\
IRAS 06206-6315* & 437.6 & 5 & 12.23 & 2.41 \\
Mrk 463 & 235.9 & 5 & 11.79 & 1.97 \\
IRAS 19254-7245* & 293.8 & 5 & 12.09 & 2.27 \\
IRAS 15462-0450* & 478.2 & 5 & 12.24 & 2.42 \\
IRAS 20087-0308* & 509.0 & 5 & 12.42 & 2.60 \\
IRAS 13451+1232* & 586.6 & 5 & 12.32 & 2.50 \\
IRAS 00188-0856* & 623.3 & 5 & 12.39 & 2.57 \\
IRAS 12071-0444* & 623.3 & 5 & 12.41 & 2.59 \\
IRAS 03158+4227* & 655.0 & 5 & 12.63 & 2.81 \\
IRAS 16090-0139* & 655.0 & 5 & 12.55 & 2.73 \\
IRAS 10378+1109* & 665.6 & 5 & 12.31 & 2.49 \\
IRAS 07598+6508* & 729.8 & 5 & 12.50 & 2.68 \\
IRAS 03521+0028* & 751.3 & 5 & 12.52 & 2.70 \\
Mrk 1014* & 811.1 & 5 & 12.62 & 2.80 \\
\hline
\end{tabular}

